ALEA, Lat. Am. J. Probab. Math. Stat. 17, 545-587 (2020)

DOI: $10.30757 /$ ALEA.v17-22

\title{
On Nonlinear Rough Paths
}

\section{David Nualart and Panqiu Xia}

Department of Mathematics, University of Kansas

1460 Jayhawk Blvd., Lawrence, Kansas, USA

E-mail address: nualart@ku.edu, pqxia@ku.edu

Abstract. In this paper, we develop the theory of nonlinear rough paths. Following the ideas of Lyons and Gubinelli, we define the nonlinear rough integral $\int_{s}^{t} W\left(d r, Y_{r}\right)$, where $W$ and $Y$ are only $\alpha$-Hölder continuous in time with $\alpha \in\left(\frac{1}{3}, \frac{1}{2}\right]$. Also, we study the Kunita-type equation $Y_{t}=\xi+\int_{0}^{t} W\left(d r, Y_{s}\right)$, obtaining the local and global existence and uniqueness of the solution under suitable sufficient conditions. As an application, we study transport equations with rough vector fields and observe that the classical solution formula for smooth and Young's cases does not provide a solution to the rough equation. Indeed this formula satisfies a transport equation with additional compensator terms (see (1.7)).

\section{Introduction}

Nonlinear integrals in the sense of Young have been studied in recent years (see e.g. Catellier and Gubinelli, 2016, Chouk and Gubinelli, 2015, 2014 and Hu and Lê, 2017). In these papers, the authors consider the following nonlinear integral

$$
\mathcal{I}_{s, t}=\int_{s}^{t} W\left(d r, Y_{r}\right),
$$

where $W$ is a function on $[0, T] \times \mathbb{R}^{d}$ with values in $\mathbb{R}^{d}$, that is $\tau$-Hölder continuous in time and $\lambda$-Hölder continuous in space, and $Y:[0, T] \rightarrow \mathbb{R}^{d}$ is $\gamma$-Hölder continuous. Under the assumption $\tau+\lambda \gamma>1$, the nonlinear integral (1.1) is well-defined in the sense of Young (see Young, 1936). That is, $\mathcal{I}_{s, t}$ is the limit of the following linear approximations as $|\pi| \rightarrow 0$

$$
\sum_{k=1}^{n} W_{t_{k-1}, t_{k}}\left(Y_{t_{k-1}}\right):=\sum_{k=1}^{n}\left[W\left(t_{k}, Y_{t_{k-1}}\right)-W\left(t_{k-1}, Y_{t_{k-1}}\right)\right]
$$

Received by the editors August 23th, 2019; accepted May 7th, 2020.

2010 Mathematics Subject Classification. 60H10, 60H15, 34G20.

Key words and phrases. Nonlinear rough paths, controlled rough paths, nonlinear rough integrals, rough differential equations, Itô's formula, rough partial differential equations.

David Nualart was supported by the NSF grant DMS 1811181. 
where $\pi=\left(s=t_{0}<t_{1}<\cdots<t_{n}=t\right)$ is a partition of the interval $[s, t]$ and $|\pi|:=\max _{1 \leq k \leq n}\left|t_{k}-t_{k-1}\right|$. As an example, one can define a pathwise nonlinear integral of the form (1.1), where $W$ is a fractional Brownian sheet with Hurst parameters $H_{0} \in\left(\frac{1}{2}, 1\right)$ in time and $H_{1}=\cdots=H_{d}=H$ in space, such that $H_{0}+\frac{1}{2} H>1$, and $Y$ is a $d$-dimensional standard Brownian motion. By applying this theory of nonlinear Young's integrals, Hu and Lê (2017) studied the following transport equation with distributional vector field (see also Catellier and Gubinelli, 2016; Flandoli et al., 2010):

$$
\frac{\partial}{\partial t} u(t, x)+D u(t, x) \frac{\partial}{\partial t} W(t, x)=0,
$$

where $D$ denotes the spatial derivative operator. The existence and uniqueness of the solution to $(1.2)$ with $\mathcal{C}_{l o c}^{1+\lambda_{0}}\left(\mathbb{R}^{d} ; \mathbb{R}\right)$-valued initial condition were proved in this paper assuming that $\left(1+\lambda_{0}\right) \tau>1$. They also provided a formula for the solution:

$$
u(t, x)=h\left(Z_{t}(x)\right)
$$

where $h$ is the initial condition, $Z_{t}$ is the inverse of $Y_{t}$, and $Y$ is the solution to the following nonlinear differential equation:

$$
Y_{t}(x)=x+\int_{0}^{t} W\left(d s, Y_{s}(x)\right) .
$$

On the other hand, applying the theory of nonlinear integrals to the stochastic heat equation, Hu and Lê also gave a pathwise proof of the Feynman-Kac formula, which provides an alternative method to study this topic (see e.g. Hu et al., 2012, 2011 for a probabilistic approach).

The purpose of this paper is to extend the theory of nonlinear integrals to the case when the functions $W$ and $Y$ are rougher, that is $\tau+\lambda \gamma<1$. In this situation, Young's approach fails. The following example, inspired by the lecture notes from Zanco (see Example 3.6 of Zanco, 2016), provides a non-standard nonlinear rough path behavior in $\mathbb{R}$. For any $n \in \mathbb{Z}_{+}, t \in[0, T]$ and $x, y \in \mathbb{R}$, we define

$$
F(x, y)=e^{x y}, X_{t}^{(n)}=\frac{1}{n} \cos \left(2 \pi n^{2} t\right) \text { and } Y_{t}^{(n)}=\frac{1}{n} \sin \left(2 \pi n^{2} t\right) .
$$

Then $F\left(X_{t}^{(n)}, y\right)$ converges to 1 and $Y_{t}^{(n)}$ converges to 0 uniformly on compact sets as $n \rightarrow \infty$. On the other hand, however, the following integral

$$
\int_{0}^{1} F\left(d X_{t}^{(n)}, Y_{t}^{(n)}\right)=-\frac{1}{4} \int_{0}^{4 \pi} \exp \left(\frac{1}{2 n^{2}} \sin \left(n^{2} s\right)\right) d s \rightarrow-\pi
$$

by dominated convergence theorem, as $n \rightarrow \infty$.

In the linear situation, a useful tool to deal with the integration of rough functions is the theory of rough paths. This theory has been developed from the pioneering work of Lyons since the early nineties (see e.g. Lyons, 1994, 1998) to study $d$ dimensional dynamical systems of the form

$$
d Y_{t}=f\left(Y_{t}\right) d X_{t}, t \in[0, T],
$$

where the driven signal $X_{t}$ is $\alpha$-Hölder continuous and $\alpha \in\left(0, \frac{1}{2}\right]$. The main idea of the rough path analysis is as follows. Let $p=\left\lfloor\frac{1}{\alpha}\right\rfloor$, and let $T^{(p)}$ be a $p$-step truncated tensor algebra given by the expression

$$
T^{(p)}:=\mathbb{R} \oplus\left(\mathbb{R}^{d}\right) \oplus\left(\mathbb{R}^{d}\right)^{\otimes 2} \oplus \cdots \oplus\left(\mathbb{R}^{d}\right)^{\otimes p} .
$$


The rough path associated to $X$ is a lifting of $X$ to a $T^{(p)}$-valued function on $[0, T]^{2}$, denoted by $S^{(p)}(X)$, in such a way that when $X$ is piecewise differentiable, the function $S_{s, t}^{(p)}=\left(1, X_{s, t}^{1}, X_{s, t}^{2}, \ldots, X_{s, t}^{p}\right)$, and each component $X_{s, t}^{i}$ is the $i$ th iterated integral of $X$ on the time interval $[s, t] \subset[0, T]$. Suppose that $f$ is a smooth function, then the integral of $f(X)$ against $X$ on $[s, t]$ can be approximated by

$$
\int_{s}^{t} f\left(X_{r}\right) d X_{r} \approx f\left(X_{s}\right) X_{s, t}^{1}+f^{\prime}\left(X_{s}\right) X_{s, t}^{2}+\cdots+f^{(p-1)}\left(X_{s}\right) X_{s, t}^{p},
$$

with an error of order $O\left(|t-s|^{(p+1) \alpha}\right)$. Because $(p+1) \alpha>1$, the error term vanishes in the limit, which explains the choice $p=\left\lfloor\frac{1}{\alpha}\right\rfloor$. This allows us to define the integral by passing the limit as $|\pi| \rightarrow 0$ of the following expression

$$
\sum_{k=1}^{n} \sum_{i=0}^{p} f^{(i-1)}\left(X_{t_{k-1}}\right) X_{t_{k-1}, t_{k}}^{i}
$$

where $\pi=\left(s=t_{1}<\cdots<t_{n}=t\right)$.

Suppose that $\alpha \in\left(\frac{1}{3}, \frac{1}{2}\right]$. Gubinelli (2004) generalized the integration of "1-forms", which means the integrand is a function $f\left(X_{t}\right)$ of the driving signal, to a class of rough functions called "controlled rough paths". A controlled rough path (by $X$ ), is a function $Y:[0, T] \rightarrow \mathbb{R}^{d}$ whose increment on an interval $[s, t]$ can be written in the following way: $Y_{s, t}=Y_{s}^{\prime} X_{s, t}+R_{s, t}^{Y}$, for some $\mathbb{R}^{d} \otimes \mathbb{R}^{d}$-valued $\alpha$-Hölder continuous function $Y^{\prime}$ and some $\mathbb{R}^{d}$-valued $2 \alpha$-Hölder continuous function $R^{Y}$. In this case, the approximation of the integral is the following

$$
\int_{s}^{t} Y_{r} d X_{r} \approx Y_{s} X_{s, t}^{1}+Y_{s}^{\prime} X_{s, t}^{2}
$$

For a more detailed account on this topic, we refer the readers to the books Friz and Hairer (2014) and Lyons and Qian (2002). An alternative approach to deal with the integration of "non-1-forms" based on fractional calculus was developed in Besalú and Nualart (2011); Hu and Nualart (2009).

In the present paper, we will extend the nonlinear Young's integral to the rough case by using Gubinelli's approach, and assuming a Hölder regularity of order $\alpha \epsilon$ $\left(\frac{1}{3}, \frac{1}{2}\right]$. The paper is organized in the following way. In Section 2 we give brief review of the preliminaries about (linear) rough paths. In Section 3 we introduce a nonlinear variant of rough paths. By definition a nonlinear rough path is a pair $(W, \mathbb{W})$ such that $W(t, x)$ is a function of two variables, $(t, x) \in[0, T] \times V$, where $V$ is Banach space. The component $\mathbb{W}_{s, t}(x, y)$ should be interpreted as the integral

$$
\mathbb{W}_{s, t}(x, y)=\int_{s}^{t} D W(d r, y) W_{r, s}(x)
$$

for any $0 \leq s \leq t \leq T$, where $D W(r, y)$ denotes the Fréchet derivative of $W$ with respect to the spatial argument $y$. We also assume that $(W, \mathbb{W})$ satisfies certain properties, including $\alpha$-Hölder continuity and a version of Chen's relation. Then, a nonlinear rough integral can be approximated in the following way:

$$
\int_{s}^{t} W\left(d r, Y_{r}\right) \approx W_{s, t}(Y)+\mathbb{W}_{s, t}\left(\dot{Y}_{s}, Y_{s}\right),
$$

where $\dot{Y}$ is the Gubinelli derivative of $Y$ in the context of nonlinear rough paths. We prove that the nonlinear rough integral is a nonlinear controlled rough path and we establish some properties of nonlinear rough integrals. 
In Section 4, we consider the following rough differential equation (RDE):

$$
Y_{t}=\xi+\int_{0}^{t} W\left(d r, Y_{r}\right)
$$

where $(W, \mathbb{W})$ is an $\alpha$-Hölder nonlinear rough path. Local and global existence and uniqueness of the solution to the RDE (1.6) are proved in this section. We also obtain some estimates for the solution to this equation. This type of RDEs was previously studied by Bailleul and his collaborators (see e.g. Bailleul and Catellier, 2017; Bailleul et al., 2017; Bailleul and Riedel, 2019) under some boundedness assumption of $W$. Here, we study this equation via a different approach, and improve their results removing boundedness conditions.

Another approach to equation (1.6) was introduced in the papers Brault and Lejay $(2019 a, 2018,2019 b)$. The authors introduced the almost flow $\phi_{s, t}(x)$. In comparison with our setting, $\phi_{s, t}(x)$ is equivalent to $W_{s, t}(x)+\mathbb{W}_{s, t}(x, x)+x$. Then without the analysis of the rough integrals, a solution to equation (1.6) can be constructed as the limit of the following iterations over partitions $\pi=\left(0=t_{0}<\right.$ $\left.t_{1}<\cdots<t_{n}=t\right)$

$$
\phi_{t_{n-1}, t_{n}} \circ \cdots \circ \phi_{t_{1}, t_{0}}(\xi), \quad \text { as }|\pi| \rightarrow 0 .
$$

In Section 5 , we study nonlinear rough paths as function space-valued linear rough paths. Then, we prove that under some assumptions, these two approaches are equivalent. Despite this, we still prefer to keep the analysis in Sections 3 and 4. Firstly, the approach to define nonlinear rough paths in Section 3 is more intuitive than the latter method based on abstract spaces. Additionally, in order to interpret nonlinear rough paths as function space-valued linear rough paths, a stronger assumption is required, namely, the existence of the integral $\mathcal{W}_{s, t}=$ $\int_{s}^{t} W_{s, r} \otimes d W_{r}$, whereas, in Sections 3 and 4 , we only need to define the integral $\mathbb{W}_{s, t}(x, y)=\int_{s}^{t} D W(d r, y) W_{r, s}(y)$.

Section 6 contains some applications of nonlinear rough paths. In Section 6.1, we provide a generalized Itô-type formula for (nonlinear) controlled rough paths. In Section 6.2 we analyze the gradient flow of the following equation with spatial parameter,

$$
Y_{t}(x)=x+\int_{0}^{t} W\left(d r, Y_{r}(x)\right),
$$

where $x \in \mathbb{R}^{d}$ and $W:[0, T] \times \mathbb{R}^{d} \rightarrow \mathbb{R}^{d}$ is a nonlinear rough path. We will prove that under some assumptions, $Y_{t}(x)$ is differentiable in $x$. In addition for every $(t, x) \in[0, T] \times \mathbb{R}^{d}$, the gradient $D Y_{t}(x)$ is an invertible matrix. Thus, for any fixed $t \in[0, T]$, by the Inverse Function Theorem in $\mathbb{R}^{d}$, there exists $Z_{t}: \mathbb{R}^{d} \rightarrow \mathbb{R}^{d}$ such that $Z_{t}\left(Y_{t}(x)\right)=Y_{t}\left(Z_{t}(x)\right)=x$ for all $x \in \mathbb{R}^{d}$. Assume that $h \in \mathcal{C}_{l o c}^{4}\left(\mathbb{R}^{d} ; \mathbb{R}\right)$. Because the structure of $W$ here is rougher than in Young's case, it turns out that $h\left(Z_{t}(x)\right)$ does not satisfies the transport equation (1.2). In Section 6.3 we will prove that $h\left(Z_{t}(x)\right)$ is indeed the solution to the following transport equation with compensators

$$
\begin{aligned}
\frac{\partial}{\partial t} u(t, x) & +D u(t, x) \frac{\partial W(t, x)}{\partial t}=\frac{1}{2} D u(t, x) \frac{\partial\left\langle\langle D W(x), W(x)\rangle_{0, t}\right.}{\partial t} \\
& +\frac{1}{2} D u(t, x) \frac{\partial\langle W(x), D W(x)\rangle_{0, t}}{\partial t}+\frac{1}{2} D^{2} u(t, x) \frac{\partial\langle W(x)\rangle_{0, t}}{\partial t} .
\end{aligned}
$$

Furthermore, the solution is unique in the space $\mathcal{C}_{l o c}^{\alpha, 3}\left([0, T] \times \mathbb{R}^{d} ; \mathbb{R}\right)$. A similar transport equation with rough vector field was also studied in Catellier (2016). 


\section{Preliminaries}

Fix a time interval $[0, T]$. Assume that $\alpha \in\left(\frac{1}{3}, \frac{1}{2}\right]$. Let $V$ and $K$ be Banach spaces. We follow the construction of Friz and Hairer (2014, Chapters 2, 4) to introduce the basic framework of the theory of (linear) rough paths.

Definition 2.1. (i) $\mathcal{C}^{\alpha}([0, T] ; V)$ is the space of functions on $[0, T]$ taking values in $V$ such that the following $\alpha$-Hölder seminorm is finite

$$
\|\Phi\|_{\alpha}:=\sup _{s \neq t \in[0, T]} \frac{\left\|\Phi_{s, t}\right\|_{V}}{|t-s|^{\alpha}}
$$

where $\Phi_{s, t}:=\Phi_{t}-\Phi_{s}$.

(ii) $\mathcal{C}_{2}^{\alpha}\left([0, T]^{2} ; K\right)$ is the space of functions on $[0, T]^{2}$ taking values in $K$ and such that the following $\alpha$-Hölder seminorm is finite

$$
\|\Psi\|_{\alpha}:=\sup _{s \neq t \in[0, T]} \frac{\left\|\Psi_{s, t}\right\|_{K}}{|t-s|^{\alpha}} .
$$

A $V$-valued rough path, introduced below, is defined as a pair of a rough function and a double integral term.

Definition 2.2. The space of rough paths $\mathscr{C}^{\alpha}([0, T] ; V)$ is the collection of pairs $\mathbf{X}=(X, \mathbb{X})$ satisfying the following properties:

(i) $X \in \mathcal{C}^{\alpha}([0, T] ; V)$.

(ii) $\mathbb{X} \in \mathcal{C}_{2}^{2 \alpha}\left([0, T]^{2} ; V \otimes V\right)$, where $V \otimes V$ is the tensor product space equipped with the projective norm.

(iii) $(X, \mathbb{X})$ satisfies Chen's relation: for all $(s, u, t) \in[0, T]^{3}$,

$$
\mathbb{X}_{s, t}-\mathbb{X}_{s, u}-\mathbb{X}_{u, t}=X_{s, u} \otimes X_{u, t} .
$$

Here $\mathbb{X}$ has to be interpreted as a version of the following double integral:

$$
\int_{s}^{t} X_{s, r} \otimes d X_{r}=\int_{s}^{t} \int_{s}^{r} d X_{u} \otimes d X_{r}:=\mathbb{X}_{s, t} .
$$

Let $X \in \mathcal{C}^{\alpha}([0, T] ; V)$. We define rough paths controlled by $X$ as follows:

Definition 2.3 (Definition 4.6 of Friz and Hairer, 2014). Let $X \in \mathcal{C}^{\alpha}([0, T] ; V)$. An element $Y \in \mathcal{C}^{\alpha}([0, T] ; K)$ is said to be controlled by $X$, if there exist functions $Y^{\prime} \in \mathcal{C}^{\alpha}([0, T] ; \mathcal{L}(V ; K))$ and $R^{Y} \in \mathcal{C}_{2}^{2 \alpha}\left([0, T]^{2} ; K\right)$, such that

$$
Y_{s, t}=Y_{s}^{\prime}\left(X_{s, t}\right)+R_{s, t}^{Y}
$$

for any $s, t \in[0, T]$. Here $\mathcal{L}(V ; K)$ denotes the space of continuous linear operators from $V$ to $K$ equipped with the operator norm. The function $Y^{\prime}$ is called the Gubinelli derivative of $Y$.

Denote by $\mathscr{D}_{X}^{2 \alpha}(K)$ the space of such pairs $\left(Y, Y^{\prime}\right)$. With an abuse of notations, we sometimes write $Y \in \mathscr{D}_{X}^{2 \alpha}(K)$ instead of $\left(Y, Y^{\prime}\right) \in \mathscr{D}_{X}^{2 \alpha}(K)$.

Suppose that $X \in \mathcal{C}^{\alpha}([0, T] ; V)$ and $\left(Y, Y^{\prime}\right) \in \mathscr{D}_{X}^{2 \alpha}(\mathcal{L}(V ; K))$. Then, $Y^{\prime}$ takes values in $\mathcal{L}(V ; \mathcal{L}(V ; K))$, which can be identified with $\mathcal{L}(V \otimes V ; K)$. The next theorem defines a version of the (linear) rough integral. 
Theorem 2.4 (Theorem 4.10 (a) of Friz and Hairer, 2014). Let $\mathbf{X}=(X, \mathbb{X}) \epsilon$ $\mathscr{C}^{\alpha}([0, T] ; V)$. Suppose that $\left(Y, Y^{\prime}\right) \in \mathscr{D}_{X}^{2 \alpha}(\mathcal{L}(V ; K))$. Then the following "compensated Riemann-Stieltjes sum"

$$
\sum_{k=1}^{n} \Xi_{t_{k}, t_{k-1}}:=\sum_{k=1}^{n}\left[Y_{t_{k-1}}\left(X_{t_{k-1}, t_{k}}\right)+Y_{t_{k-1}}^{\prime}\left(\mathbb{X}_{t_{k-1}, t_{k}}\right)\right],
$$

converges as $|\pi| \rightarrow 0$, where $\pi=\left(s=t_{1}<t_{2}<\cdots<t_{n}=t\right)$ and $|\pi|=\max _{1 \leq k \leq n} \mid t_{k}-$ $t_{k-1} \mid$. Denote by $\mathcal{J}_{s, t}(\Xi)$ the limit of (2.4). Then, $\mathcal{J}_{s, t}(\Xi)$ is additive, that is $\mathcal{J}_{s, t}(\Xi)=\mathcal{J}_{s, u}(\Xi)+\mathcal{J}_{u, t}(\Xi)$ for any $0 \leq s<u<t \leq T$. Moreover, the following estimate is satisfied for all $0 \leq s<t \leq T$,

$$
\left\|\mathcal{J}_{s, t}(\Xi)-\Xi_{s, t}\right\|_{K} \leq k_{\alpha}\left(\|X\|_{\alpha}\left\|R^{Y}\right\|_{2 \alpha}+\|\mathbb{X}\|_{2 \alpha}\left\|Y^{\prime}\right\|_{\alpha}\right)|t-s|^{3 \alpha},
$$

where $k_{\alpha}=\left(1-2^{1-3 \alpha}\right)^{-1}$. By definition, the rough integral of $Y$ against $\mathbf{X}=(X, \mathbb{X})$ is defined as follows, for all $0 \leq s<t \leq T$,

$$
\int_{s}^{t} Y_{r} d \mathbf{X}_{r}:=\mathcal{J}_{s, t}(\Xi)
$$

Similarly we can define the rough integral $\int_{s}^{t} Y_{r} \otimes d \mathbf{X}_{r} \in V_{1} \otimes V_{2}$, for any $\mathbf{X}=$ $(X, \mathbb{X}) \in \mathscr{C}^{\alpha}\left([0, T] ; V_{1}\right)$ and $\left(Y, Y^{\prime}\right) \in \mathscr{D}_{X}^{2 \alpha}\left(V_{2}\right)$. Theorem 2.4 can be proved by using the following Sewing Lemma. In this case, $\gamma=3 \alpha>1$ and $k_{\alpha}$ comes from inequality (2.7) below. The Sewing Lemma is cited from Lemma 2.1 of Feyel and de La Pradelle (2006), see also Gubinelli (2004). It will also be used later in the theory of nonlinear rough paths.

Lemma 2.5 (Sewing Lemma). Let $\beta \in(0,1]$, and let $\Xi \in \mathcal{C}_{2}^{\beta}\left([0, T]^{2} ; K\right)$. Suppose there exist $C>0$ and $\gamma>1$ such that the following inequality holds:

$$
\|\delta \Xi(s, u, t)\|_{K}:=\left\|\Xi_{s, t}-\Xi_{s, u}-\Xi_{u, t}\right\|_{K} \leq C|t-s|^{\gamma},
$$

for any $0 \leq s \leq u \leq t \leq T$. Then there exists a unique (up to an additive constant) function $\mathcal{J}(\Xi) \in \mathcal{C}^{\beta}([0, T] ; V)$, such that the following inequality holds

$$
\left\|\mathcal{J}_{s, t}(\Xi)-\Xi_{s, t}\right\|_{K}=\left\|\mathcal{J}_{t}(\Xi)-\mathcal{J}_{s}(\Xi)-\Xi_{s, t}\right\|_{K} \leq\left(1-2^{1-\gamma}\right)^{-1} C|t-s|^{\gamma} .
$$

Moreover, $\mathcal{J}_{s, t}(\Xi)$ can be represented as follows,

$$
\mathcal{J}_{s, t}(\Xi)=\lim _{|\pi| \rightarrow 0} \sum_{k=1}^{n} \Xi_{t_{k-1}, t_{k}},
$$

where $\pi=\left(s=t_{0}<t_{1}<\cdots<t_{n}=t\right)$ and the limit is independent of the choice of $\pi$.

The next proposition shows that the rough integral is controlled by $X$.

Proposition 2.6 (Theorem 4.10 (b) of Friz and Hairer, 2014). Suppose that $(X, \mathbb{X})$ $\in \mathscr{C}^{\alpha}([0, T] ; V)$ and $\left(Y, Y^{\prime}\right) \in \mathscr{D}_{X}^{2 \alpha}(\mathcal{L}(V ; K))$. Let

$$
Z_{t}=\int_{0}^{t} Y_{r} d \mathbf{X}_{r}
$$

Then, $Z$ is an $\alpha$-Hölder continuous function taking values in $K$. Moreover $Z$ is controlled by $X$ with $Y$ as a Gubinelli derivative.

In the next proposition, we define the integration of two controlled rough paths.

Proposition 2.7. Let $V, K_{1}$ and $K_{2}$ be Banach spaces. Suppose that $\mathbf{X}=(X, \mathbb{X}) \epsilon$ $\mathscr{C}^{\alpha}([0, T] ; V)$ and $\left(Y, Y^{\prime}\right) \in \mathscr{D}_{X}^{2 \alpha}\left(K_{1}\right)$. 
(i) [Remark 4.11 of Friz and Hairer, 2014] Suppose that $\left(Z, Z^{\prime}\right) \in \mathscr{D}_{X}^{2 \alpha}\left(K_{2}\right)$. The following limit exists

$$
\lim _{|\pi| \rightarrow 0} \sum_{k=1}^{n}\left[Z_{t_{k-1}} \otimes Y_{t_{k-1}, t_{k}}+\left(Z_{t_{k-1}}^{\prime} \otimes Y_{t_{k-1}}^{\prime}\right)\left(\mathbb{X}_{t_{k-1}, t_{k}}\right)\right],
$$

where $\pi=\left(s=t_{0}<t_{1}<\cdots<t_{n}=t\right)$ and defines the integral $\int_{s}^{t} Z_{r} \otimes d Y_{r}$.

(ii) [Proposition 7.1 of Friz and Hairer, 2014] Let $\mathbb{Y}:[0, T]^{2} \rightarrow K_{1} \otimes K_{1}$ be given by

$$
\mathbb{Y}_{s, t}=\int_{s}^{t} Y_{r} \otimes d Y_{r}-Y_{s} \otimes Y_{s, t}
$$

and the integral in $(2.10)$ is defined by (2.9). Then, $\mathbf{Y}:=(Y, \mathbb{Y})$ is a rough path. Suppose that $\left(Z, \widetilde{Z}^{\prime}\right) \in \mathscr{D}_{Y}^{2 \alpha}\left(K_{2}\right)$. Let $Z_{t}^{\prime}=\widetilde{Z}_{t}^{\prime} Y_{t}^{\prime}$ for all $t \in[0, T]$. Then, $\left(Z, Z^{\prime}\right) \in \mathscr{D}_{X}^{2 \alpha}\left(K_{2}\right)$. In addition, the following equality holds

$$
\int_{s}^{t} Z_{r} \otimes d \mathbf{Y}_{r}=\int_{s}^{t} Z_{r} \otimes d Y_{r},
$$

where the integral on the left-hand side is in the sense of Theorem 2.4, and the integral on the right-hand side is in the sense of (2.9).

Remark 2.8. Assume the conditions of Proposition 2.7 (i) where $K_{2}=\mathcal{L}\left(K_{1} ; K\right)$. Then,

$$
\int_{s}^{t} Z_{r} d Y_{r}:=\lim _{|\pi| \rightarrow 0} \sum_{k=1}^{n}\left[Z_{t_{k-1}}\left(Y_{t_{k-1}, t_{k}}\right)+\left(Z_{t_{k-1}}^{\prime} Y_{t_{k-1}}^{\prime}\right) \mathbb{X}_{t_{k-1}, t_{k}}\right]
$$

and

$$
\int_{s}^{t} d Z_{r}\left(Y_{r}\right):=\lim _{|\pi| \rightarrow 0} \sum_{k=1}^{n}\left[Z_{t_{k-1}, t_{k}}\left(Y_{t_{k-1}}\right)+\left(Z_{t_{k-1}}^{\prime} Y_{t_{k-1}}^{\prime}\right) \mathbb{X}_{t_{k-1}, t_{k}}^{*}\right],
$$

are well-defined, where $\pi=\left(s=t_{0}<t_{1}<\cdots<t_{n}=t\right),\left(Z_{t}^{\prime} Y_{t}^{\prime}\right): V \otimes V \rightarrow K$ is given by

$$
\left(Z_{t}^{\prime} Y_{t}^{\prime}\right)(x, y)=Z_{t}^{\prime}(x)\left[Y_{t}^{\prime}(y)\right]
$$

and $*$ denotes the transpose operator on the tensor product space $V \otimes V$, namely, $(x \otimes y)^{*}=y \otimes x$.

Next, we define the "quadratic compensator" as follows (see e.g. (2.7) of Keller and Zhang, 2016 for an equivalent definition in finite dimensions). It will be used in Section 6.

Definition 2.9. Let $\mathbf{X}=(X, \mathbb{X}) \in \mathscr{C}^{\alpha}([0, T] ; V)$. Suppose that $\left(Y, Y^{\prime}\right) \in \mathscr{D}_{X}^{2 \alpha}\left(K_{1}\right)$ and $\left(Z, Z^{\prime}\right) \in \mathscr{D}_{X}^{2 \alpha}\left(K_{2}\right)$.

(i) The quadratic compensator $\langle X\rangle$ is a function on $[0, T]^{2}$ with values in $V \otimes V$ given by

$$
\langle X\rangle_{s, t}:=X_{s, t} \otimes X_{s, t}-2 \mathbb{X}_{s, t} .
$$

(ii) The quadratic compensator $\langle Z, Y\rangle:[0, T]^{2} \rightarrow K_{2} \otimes K_{1}$ is given by

$$
\langle Z, Y\rangle_{s, t}:=Z_{s, t} \otimes Y_{s, t}-2 \int_{s}^{t} Z_{s, r} \otimes d Y_{r} .
$$


Remark 2.10. (i) Similar as the quadratic variation of Itô processes, the following equality holds:

$$
\langle Y, Z\rangle_{s, t}=\int_{s}^{t} Y_{r}^{\prime} \otimes Z_{r}^{\prime} d\langle X\rangle_{r} .
$$

(ii) Particularly, if $K_{2}=\mathcal{L}\left(K_{1} ; K\right)$, we write

$$
\langle Z, Y\rangle_{s, t}:=Z_{s, t} Y_{s, t}-2 \int_{s}^{t} Z_{s, r} d Y_{r}
$$

and

$$
\left\langle\langle Y, Z\rangle_{s, t}:=Z_{s, t} Y_{s, t}-2 \int_{s}^{t} d Z_{r}\left(Y_{s, r}\right) .\right.
$$

(iii) It is easy to verify that $\langle X\rangle \in \mathcal{C}_{2}^{2 \alpha}([0, T] ; V \otimes V)$. Similarly, $\langle Y, Z\rangle,\langle Z, Y\rangle$, $\langle Y Y, Z\rangle$ and $\langle\langle Z, Y\rangle$ are also $2 \alpha$-Hölder continuous in corresponding spaces.

Finally, we finish this section by introducing the following Taylor's theorem (see e.g. Theorem 4.C of Zeidler, 1995) for Banach space valued functions. It will be used frequently in estimating residuals.

Theorem 2.11 (Taylor's Theorem). Let $V$ and $K$ be Banach spaces. Assume that the map $\phi: V \rightarrow K$ is $\mathcal{C}^{n}$ in the sense of Fréchet differentiability. Then for any $v, h \in V$, the following generalized Taylor formula holds

$$
\phi(v+h)=\phi(v)+\sum_{k=1}^{n-1} \frac{1}{k !} D^{k} \phi(v) h^{\otimes k}+R_{n},
$$

where the residual $R_{n}$ satisfies the following inequality

$$
\left\|R_{n}\right\|_{K} \leq \frac{1}{n !} \sup _{0 \leq \tau \leq 1}\left\|D^{k} \phi(u+\tau h) h^{\otimes n}\right\|_{K} .
$$

\section{Nonlinear rough integrals}

3.1. Definitions. Fix a time interval $[0, T]$. Suppose that $\alpha \in\left(\frac{1}{3}, \frac{1}{2}\right]$. In this section, we aim to define the following nonlinear integral:

$$
\int_{s}^{t} W\left(d r, Y_{r}\right)
$$

Here $W$ is $\alpha$-Hölder continuous in time, and differentiable in space, and $Y$ is $\alpha$ Hölder continuous. The idea is as follows. Assume that $Y$ is controlled by $W$, that is $Y_{s, t}=W_{s, t}\left(\dot{Y}_{s}\right)+O\left(|t-s|^{2 \alpha}\right)$. Then, we approximate the nonlinear integral by the following expression:

$$
\begin{aligned}
\int_{s}^{t} W\left(d r, Y_{r}\right) & \approx \int_{s}^{t} W\left(d r, Y_{s}\right)+\int_{s}^{t} D W\left(d r, Y_{s}\right) Y_{r, s} \\
& \approx \int_{s}^{t} W\left(d r, Y_{s}\right)+\int_{s}^{t} D W\left(d r, Y_{s}\right) W_{s, r}\left(\dot{Y}_{s}\right) \\
& =W_{s, t}\left(Y_{s}\right)+\left.\int_{s}^{t} D W(d r, y) W_{s, r}(x)\right|_{(x, y)=\left(\dot{Y}_{s}, Y_{s}\right)},
\end{aligned}
$$

with the error of order $O\left(|t-s|^{3 \alpha}\right)$. This allows us to pass to the limit as $|\pi| \rightarrow 0$ in the following expression

$$
\sum_{k=1}^{n}\left[W_{t_{k-1}, t_{k}}\left(Y_{t_{k-1}}\right)+\left.\int_{t_{k-1}}^{t_{k}} D W(d r, y) W_{t_{k-1}, r}(x)\right|_{(x, y)=\left(\dot{Y}_{t_{k-1}}, Y_{t_{k-1}}\right)}\right]
$$


where $\pi=\left(s=t_{0}<t_{1}<\cdots<t_{n}=t\right)$. The limit is the desired nonlinear integral.

To this end, we need to introduce the following definitions. Let $n$ be any nonnegative integer. We denote by $\mathcal{I}_{n}$ the set of all multi-indexes $\beta_{n}$ of length $n+1$. That is, $\boldsymbol{\beta}_{n}=\left(\beta_{0}, \ldots, \beta_{n}\right)$, where $\beta_{0}, \ldots, \beta_{n}$ are nonnegative real numbers. These multi-indexes will be used to characterize the growth of a function and its spatial derivatives.

Definition 3.1. (i) $\mathcal{C}^{\alpha, \beta_{n}}([0, T] \times V ; K)$ is the space of functions such that the following seminorm is finite:

$$
\|\Phi\|_{\alpha, \boldsymbol{\beta}_{n}}:=\sum_{k=0}^{n} \sup _{\substack{s \neq t \in[0, T] \\ x \in V}} \frac{\left\|D^{k} \Phi_{s, t}(x)\right\|_{\mathfrak{L}_{k}(V ; K)}}{|t-s|^{\alpha}\left(1+\|x\|_{V}\right)^{\beta_{k}}},
$$

where $D^{k}$ is the $k$-th Fréchet derivative operator, and $\mathfrak{L}_{k}(V ; K)$ is the corresponding space of linear operators. That is, $\mathfrak{L}_{0}(V ; K)=K$ and $\mathfrak{L}_{k}(V ; K)=$ $\mathcal{L}\left(V ; \mathfrak{L}_{k-1}(V ; K)\right)$ for all $k=1,2, \ldots, n$.

(ii) $\mathcal{C}_{2}^{\alpha, \beta_{n}^{1}, \beta_{n}^{2}}\left([0, T]^{2} \times V^{2} ; K\right)$ is the space of functions such that the following seminorm is finite:

$$
\|\Psi\|_{\alpha, \boldsymbol{\beta}_{n}^{1}, \beta_{n}^{2}}:=\sum_{k=0}^{n} \sup _{\substack{s \neq t \in[0, T] \\ \mathbf{x}=\left(x_{1}, x_{2}\right) \in V^{2}}} \frac{\left\|D^{k} \Psi_{s, t}(\mathbf{x})\right\|_{\mathfrak{L}_{k}\left(V^{2} ; K\right)}}{|t-s|^{\alpha}\left(1+\left\|x_{1}\right\|_{V}\right)^{\beta_{k}^{1}\left(1+\left\|x_{2}\right\|_{V}\right)^{\beta_{k}^{2}}},}
$$

where $\mathfrak{L}_{k}\left(V^{2} ; K\right)$ are the corresponding linear spaces of derivatives and the product space $V^{2}$ is treated as a Banach space equipped with the norm $\|\mathbf{x}\|_{V^{2}}=$ $\left\|x_{1}\right\|_{V}+\left\|x_{2}\right\|_{V}$.

For any positive integer $m \leq n$, we write $\beta_{n}-m=\left(\beta_{0}, \ldots, \beta_{n-m}\right)$. Then, by definition, it is easy to verify that $\mathcal{C}^{\alpha, \boldsymbol{\beta}_{n}}([0, T] \times V ; K) \subset \mathcal{C}^{\alpha, \boldsymbol{\beta}_{n}-m}([0, T] \times V ; K)$. Let $\boldsymbol{\beta}_{n}, \widetilde{\boldsymbol{\beta}}_{n} \in \mathcal{I}_{n}$, we write $\boldsymbol{\beta}_{n} \leq \widetilde{\boldsymbol{\beta}}_{n}$ if $\beta_{k} \leq \widetilde{\beta}_{k}$ for all $k=0, \ldots, n$. Then, $\mathcal{C}^{\alpha, \boldsymbol{\beta}_{n}}([0, T] \times$ $V ; K) \subset \mathcal{C}^{\alpha, \widetilde{\boldsymbol{\beta}}_{n}}([0, T] \times V ; K)$ if $\boldsymbol{\beta}_{n} \leq \widetilde{\boldsymbol{\beta}}_{n}$. The space $\mathcal{C}_{2}^{\alpha, \boldsymbol{\beta}_{n}^{1}, \boldsymbol{\beta}_{n}^{2}}\left([0, T]^{2} \times V^{2} ; K\right)$ also has a similar property. Given a multi-index $\beta_{n}$ where $n \geq 1$, we make use of the following notations:

$$
\boldsymbol{\beta}_{n-1}^{*}=\left(\beta_{0}^{*}, \ldots, \beta_{n-1}^{*}\right) \text { and } \beta_{n-1}^{* *}=\left(\beta_{0}^{* *}, \ldots, \beta_{n-1}^{* *}\right),
$$

where $\beta_{k}^{*}:=\max \left\{\beta_{0}, \ldots, \beta_{k}\right\}$ and $\beta_{k}^{* *}:=\max \left\{\beta_{1}, \ldots, \beta_{k+1}\right\}$ for all $0 \leq k \leq n-1$.

Given multi-indexes $\beta_{2}, \beta_{1}^{*}$ and $\beta_{1}^{* *}$, let $\Phi \in \mathcal{C}^{\alpha, \beta_{2}}([0, T] \times V ; K)$ and let $\Psi \epsilon$ $\mathcal{C}_{2}^{\alpha, \boldsymbol{\beta}_{1}^{*}, \boldsymbol{\beta}_{1}^{* *}}\left([0, T]^{2} \times V^{2} ; K\right)$. We make use of the following notations: $\mathscr{R}^{\Phi}:[0, T] \times$ $V^{2} \rightarrow K$ and $\mathscr{D}^{\Psi}:[0, T]^{2} \times V^{4} \rightarrow K$ are given by

$$
\mathscr{R}_{t}^{\Phi}(x, y):=\Phi_{t}(y)-\Phi_{t}(x)-D \Phi_{t}(x)(y-x), x, y \in V
$$

and

$$
\mathscr{D}_{s, t}^{\Psi}(\mathbf{x}, \mathbf{y})=\Psi_{s, t}(\mathbf{y})-\Psi_{s, t}(\mathbf{x}), \mathbf{x}, \mathbf{y} \in V^{2} .
$$

The following lemma provides the estimates for $\mathscr{R}^{\Phi}, \mathscr{D}^{\Psi}$ and their derivatives. It will be used in the proof of the stability of nonlinear rough integrals.

Lemma 3.2. Suppose that $\mathscr{R}^{\Phi}$ and $\mathscr{D}^{\Psi}$ are given as in (3.4) and (3.5), respectively. Then, for any $x, y \in V$, and $\mathbf{x}=\left(x_{1}, x_{2}\right), \mathbf{y}=\left(y_{1}, y_{2}\right) \in V^{2}$, the following inequalities 
are satisfied:

$$
\begin{aligned}
\left\|\mathscr{R}_{s, t}^{\Phi}(x, y)\right\|_{K} \leq & \frac{1}{2}\|\Phi\|_{\alpha, \boldsymbol{\beta}_{2}}\left(1+\|x\|_{V}+\|y\|_{V}\right)^{\beta_{2}}\|y-x\|_{V}^{2}|t-s|^{\alpha} \\
\left\|\mathscr{D}_{s, t}^{\Psi}(\mathbf{x}, \mathbf{y})\right\|_{K} \leq & \|\Psi\|_{\alpha, \boldsymbol{\beta}_{1}^{1}, \boldsymbol{\beta}_{1}^{2}}\left(1+\left\|x_{1}\right\|_{V}+\left\|y_{1}\right\|_{V}\right)^{\beta_{1}^{1}}\left(1+\left\|x_{2}\right\|_{V}+\left\|y_{2}\right\|_{V}\right)^{\beta_{1}^{2}} \\
& \times\|\mathbf{y}-\mathbf{x}\|_{V^{2}}|t-s|^{\alpha} .
\end{aligned}
$$

If furthermore $\Phi \in \mathcal{C}^{\alpha, \beta_{3}}([0, T] \times V ; K)$ and $\Psi \in \mathcal{C}_{2}^{\alpha, \beta_{2}^{1}, \beta_{2}^{2}}\left([0, T]^{2} \times V^{2} ; K\right)$, then, for all $\mathbf{z}^{1}, \mathbf{z}^{2} \in V^{2}$, the following inequalities are satisfied:

$$
\begin{aligned}
\left\|D \mathscr{R}_{s, t}^{\Phi}(x, y)\left(z_{1}, z_{2}\right)\right\|_{K} \leq & \|\Phi\|_{\alpha, \beta_{3}}\left(1+\|x\|_{V}+\|y\|_{V}\right)^{\beta_{2} \vee \beta_{3}} \\
& \times\left[\|y-x\|_{V}^{2}\left\|z_{2}\right\|_{V}+\|y-x\|_{V}\left\|z_{1}-z_{2}\right\|_{V}\right]|t-s|^{\alpha}
\end{aligned}
$$

and

$$
\begin{aligned}
\| D \mathscr{D}_{s, t}^{\Psi}(\mathbf{x}, \mathbf{y}) & \left(\mathbf{z}^{1}, \mathbf{z}^{2}\right)\left\|_{K} \leq\right\| \Psi \|_{\alpha, \boldsymbol{\beta}_{1}^{1}, \boldsymbol{\beta}_{2}^{2}}\left(1+\left\|x_{1}\right\|_{V}+\left\|y_{1}\right\|_{V}\right)^{\beta_{1}^{1} \vee \beta_{2}^{1}} \\
& \times\left(1+\left\|x_{2}\right\|_{V}+\left\|y_{2}\right\|_{V}\right)^{\beta_{1}^{2} \vee \beta_{2}^{2}}\left[\|\mathbf{y}-\mathbf{x}\|_{V^{2}}\left\|\mathbf{z}^{2}\right\|_{V^{2}}+\left\|\mathbf{z}^{1}-\mathbf{z}^{2}\right\|_{V^{2}}\right]|t-s|^{\alpha} .
\end{aligned}
$$

Proof: Inequality (3.6) is a consequence of Taylor's Theorem 2.11 and the linearity of $D$ :

$$
\begin{aligned}
\left\|\mathscr{R}_{s, t}^{\Phi}(x, y)\right\|_{K} & \leq \frac{1}{2} \sup _{0 \leq \tau \leq 1}\left\|D^{2} \Phi_{s, t}(\tau x+(1-\tau) y)(y-x, y-x)\right\|_{K} \\
& \leq \frac{1}{2}\|\Phi\|_{\alpha, \boldsymbol{\beta}_{2}}\left(1+\|x\|_{V}+\|y\|_{V}\right)^{\beta_{2}}\|y-x\|_{V}^{2}|t-s|^{\alpha} .
\end{aligned}
$$

For inequality (3.8), we assume that $\Phi \in \mathcal{C}^{\alpha, \beta_{3}}([0, T] \times V ; K)$. Then, by differentiating $\mathscr{R}_{s, t}^{\Phi}$ on the spatial argument, for any $(x, y),\left(z_{1}, z_{2}\right) \in V^{2}$, we have

$$
\begin{aligned}
D \mathscr{R}_{s, t}^{\Phi}(x, y)\left(z_{1}, z_{2}\right)= & -D \Phi_{s, t}(x)\left(z_{1}\right)-D^{2} \Phi_{s, t}(x)\left(z_{1}, y-x\right)+D \Phi_{s, t}(x)\left(z_{1}\right) \\
& +D \Phi_{s, t}(y)\left(z_{2}\right)-D \Phi_{s, t}(x)\left(z_{2}\right) \\
= & D \Phi_{s, t}(y)\left(z_{2}\right)-D \Phi_{s, t}(x)\left(z_{2}\right)-D^{2} \Phi_{s, t}(x)\left(z_{1}, y-x\right) \\
= & D \Phi_{s, t}(y)\left(z_{2}\right)-D \Phi_{s, t}(x)\left(z_{2}\right)-D^{2} \Phi_{s, t}(x)\left(z_{2}, y-x\right) \\
& +D^{2} \Phi_{s, t}(x)\left(z_{2}-z_{1}, y-x\right) .
\end{aligned}
$$

By Taylor's Theorem 2.11 again, we can deduce that

$$
\begin{aligned}
& \left\|D \Phi_{s, t}(y)\left(z_{2}\right)-D \Phi_{s, t}(x)\left(z_{2}\right)-D^{2} \Phi_{s, t}(x)\left(z_{2}, y-x\right)\right\|_{K} \\
\leq & \frac{1}{2} \sup _{0 \leq \tau \leq 1}\left\|D^{3} \Phi_{s, t}(\tau x+(1-\tau) y)\left(z_{2}, y-x, y-x\right)\right\|_{K} .
\end{aligned}
$$

Thus inequality (3.8) is a consequence of above two inequalities. Inequalities (3.7) and (3.9) can be proved similarly.

In the rest of this paper, we focus on the case when $K=V$. A nonlinear rough path is defined as follows.

Definition 3.3. Assume that $n \geq 1$. An $\alpha$-Hölder nonlinear rough path $\mathbf{W}$ on the space $\mathcal{C}^{\alpha, \boldsymbol{\beta}_{n}}([0, T] \times V ; V)$ is defined as a pair $(W, \mathbb{W})$ that satisfies the following properties:

(i) $W \in \mathcal{C}^{\alpha, \beta_{n}}([0, T] \times V ; V)$.

(ii) $\mathbb{W} \in \mathcal{C}_{2}^{2 \alpha, \boldsymbol{\beta}_{n-1}^{*}, \boldsymbol{\beta}_{n-1}^{* *}}\left([0, T]^{2} \times V^{2} ; V\right)$, where $\boldsymbol{\beta}_{n-1}^{*}$ and $\boldsymbol{\beta}_{n-1}^{* *}$ are defined in (3.3). 
(iii) $(W, \mathbb{W})$ satisfies Chen's relation:

$$
\mathbb{W}_{s, t}(x, y)-\mathbb{W}_{s, u}(x, y)-\mathbb{W}_{u, t}(x, y)=D W_{u, t}(y)\left(W_{s, u}(x)\right),
$$

for all $(x, y) \in V^{2}$ and $s, u, t \in[0, T]$.

The collection of such rough paths is denoted by $\mathscr{C}^{\alpha, \boldsymbol{\beta}_{n}}([0, T] \times V ; V)$.

Remark 3.4. (i) In the smooth case, $\mathbb{W}$ can be interpreted as the following integral

$$
\int_{s}^{t} D W(d r, y)\left(W_{s, r}(x)\right)=\int_{s}^{t} \frac{\partial}{\partial r} D W(r, y)\left(W_{s, r}(x)\right) d r=\mathbb{W}_{s, t}(x, y) .
$$

This explains the choice of the multi-indexes $\beta_{n-1}^{*}$ and $\beta_{n-1}^{* *}$ in point (ii) of Definition 3.3. For example, assume that $W$ is twice differentiable with growth index $\beta_{2}$. Then, one can bound the growth of $\mathbb{W}_{s, t}$ as follows

$$
\begin{aligned}
& \frac{\left\|\mathbb{W}_{s, t}(x, y)\right\|_{V \otimes V}}{\left(1+\|x\|_{V}\right)^{\beta_{0}}\left(1+\|y\|_{V}\right)^{\beta_{1}}} \\
\leq & \limsup _{|\pi| \rightarrow 0} \frac{\sum_{k=1}^{n}\left|\frac{\partial}{\partial r} D W\left(t_{k-1}, y\right)\left(W_{s, t_{k-1}}(x)\right)\right|\left|t_{k}-t_{k-1}\right|}{\left(1+\|x\|_{V}\right)^{\beta_{0}}\left(1+\|y\|_{V}\right)^{\beta_{1}}} \leq\|W\|_{1, \boldsymbol{\beta}_{2}}^{2}|t-s| .
\end{aligned}
$$

By taking the derivative of $\mathbb{W}_{s, t}$, one can deduce that the growth of $D \mathbb{W}_{s, t}(x, y)$ is bounded by $\beta_{0} \vee \beta_{1}$ and $\beta_{1} \vee \beta_{2}$ in $x$ and $y$,respectively.

(ii) By definition, we can deduce that $\mathscr{C}^{\alpha, \boldsymbol{\beta}_{n}^{1}}([0, T] \times V ; V) \subset \mathscr{C}^{\alpha, \beta_{n}^{2}-m}([0, T] \times$ $V ; V)$ for all $m \in\{0, \ldots, n\}$ and $\beta_{n}^{1} \leq \beta_{n}^{2}$.

(iii) Assume that $W(t, x)=W_{t}(x)$ where $W_{t} \in \mathcal{L}(V ; V)$. Then the nonlinear rough path degenerates to the linear rough path. In this case, $D W_{t}(x)=W_{t}$ and thus

$$
\mathbb{W}_{s, t}(x, y)=\int_{s}^{t} W(d r)\left(W_{s, r}(x)\right) .
$$

Let $\mathbf{W}=(W, \mathbb{W}) \in \mathcal{C}^{\alpha, \beta_{n}}([0, T] \times V ; V)$. We make use of the notation

$$
\|\mathbf{W}\|_{\mathscr{C}_{n}}:=\|W\|_{\alpha, \boldsymbol{\beta}_{n}}+\|\mathbb{W}\|_{\alpha, \boldsymbol{\beta}_{n-1}^{*}, \boldsymbol{\beta}_{n-1}^{* *}} .
$$

Notice that $\mathscr{C}^{\alpha, \beta_{n}}([0, T] \times V ; V)$ is not a linear space with the usual addition and scalar product. Thus $\|\cdot\|_{\mathscr{C}_{n}}$ is not a seminorm in the usual sense. We introduce the pseudometric on $\mathscr{C}^{\alpha, \boldsymbol{\beta}_{n}}([0, T] \times V ; V)$ given by

$$
\varrho_{\alpha, \beta_{n}}(\mathbf{W}, \widetilde{\mathbf{W}})=\|W-\widetilde{W}\|_{\alpha, \boldsymbol{\beta}_{n}}+\|\mathbb{W}-\widetilde{\mathbb{W}}\|_{2 \alpha, \boldsymbol{\beta}_{n-1}^{*}, \boldsymbol{\beta}_{n-1}^{* *}} .
$$

Consider the following equivalent relation: $\mathbf{W} \sim \widetilde{\mathbf{W}}$ if and only if there exists $f \in \mathcal{C}^{\boldsymbol{\beta}_{n}}(V ; V)$ such that $W(t, x)-\widetilde{W}(t, x)=f(x)$ for all $(t, x) \in[0, T] \times V$. Then, $\varrho_{\alpha, \beta_{n}}$ is really a metric on the quotient space $\mathcal{C}^{\alpha, \boldsymbol{\beta}_{n}}([0, T] \times V ; V) / \sim$.

Let $W \in \mathcal{C}^{\alpha, \beta_{n}}([0, T] \times V ; V)$. Like in the linear case, we also define the space of nonlinear rough paths controlled by $W$.

Definition 3.5. The space of basic nonlinear rough paths controlled by $W$, denoted by $\mathscr{E}_{W}^{2 \alpha}$, is the collection of pairs $(Y, \dot{Y}) \in \mathcal{C}^{\alpha}([0, T] ; V) \times \mathcal{C}^{\alpha}([0, T] ; V)($ see $(2.1))$ such that, for all $s, t \in[0, T]$,

$$
Y_{s, t}=W_{s, t}\left(\dot{Y}_{s}\right)+R_{s, t}^{Y}
$$

where $R^{Y} \in \mathcal{C}_{2}^{2 \alpha}\left([0, T]^{2} ; V\right)$ (see (2.2)). The function $\dot{Y}$ above is called the Gubinelli derivative of $Y$ with respect to $W$. 
Remark 3.6. (i) In the linear case, the set of controlled rough paths is a linear space. However, in the nonlinear case, the set $\mathscr{E}_{W}^{2 \alpha}$ does not need to be a linear space with the usual addition and scalar product, because it may be not closed under these operations.

(ii) Assume that $V=\mathbb{R}$ and $W(t, x)=x W_{t}$, then the controlled rough path satisfies the following equality

$$
Y_{s, t}=\dot{Y}_{s} W_{s, t}+R_{s, t}^{Y},
$$

which coincides with the classical definition (see Definition 2.3) in the linear case.

(iii) With an abuse of notations, we sometimes write $Y \in \mathscr{E}_{W}^{2 \alpha}$ instead of $(Y, \dot{Y}) \epsilon$ $\mathscr{E}_{W}^{2 \alpha}$.

Suppose that $W, \widetilde{W} \in \mathcal{C}^{\alpha, \beta_{n}}([0, T] \times V ; V)$. Let $(Y, \dot{Y}) \in \mathscr{E}_{W}^{2 \alpha}$ and $(\widetilde{Y}, \dot{\widetilde{Y}}) \in \mathscr{E} 2 \alpha$. A "distance" between $(Y, \dot{Y})$ and $(\widetilde{Y}, \dot{\widetilde{Y}})$ is defined as follows (see e.g. Friz and Hairer, 2014, Section 4.4 for the linear case):

$$
d_{\alpha, W, \widetilde{W}}((Y, \dot{Y}),(\widetilde{Y}, \dot{\tilde{Y}}))=\|\dot{Y}-\dot{\tilde{Y}}\|_{\alpha}+\left\|R^{Y}-R^{\widetilde{Y}}\right\|_{2 \alpha} .
$$

Notice that the definition of $d_{\alpha, W, \widetilde{W}}$ does not include the term $\|Y-\widetilde{Y}\|_{\alpha}$. Indeed, this term can be estimated in terms of $d_{\alpha, W, \widetilde{W}}((Y, \dot{Y}),(\widetilde{Y}, \dot{\widetilde{Y}}))$ as it is shown in the next lemma. On the other hand, one will see in the next lemma, that $\|Y-\widetilde{Y}\|_{\alpha}$ depends also on $\|W-\widetilde{W}\|_{\alpha, \beta_{1}}$ without a factor $T^{\alpha}$. If we include $\|W-\widetilde{W}\|_{\alpha, \beta_{1}}$ in $d_{\alpha, W, \widetilde{W}}((Y, \dot{Y}),(\widetilde{Y}, \dot{\tilde{Y}}))$, the absence of this factor $T^{\alpha}$ will cause difficulties in the proof of the existence of solutions to RDEs in Section 4. For this reason, the term $\|W-\widetilde{W}\|_{\alpha, \beta_{1}}$ is not included in $d_{\alpha, W, \widetilde{W}}((Y, \dot{Y}),(\widetilde{Y}, \dot{\widetilde{Y}}))$, and it is treated independently.

Lemma 3.7. Let $W, \widetilde{W} \in \mathcal{C}^{\alpha, \beta_{1}}([0, T] \times V ; V)$. Suppose that $(Y, \dot{Y}) \in \mathscr{E}_{W}^{2 \alpha}$ and $(\widetilde{Y}, \dot{\widetilde{Y}}) \in \mathscr{E} \frac{\mathscr{E}}{\bar{W}}$. Then the following estimate holds:

$$
\begin{aligned}
\|Y-\widetilde{Y}\|_{\alpha} \leq & \left(1+\|\dot{Y}\|_{\infty}\right)^{\beta_{0}}\|W-\widetilde{W}\|_{\alpha, \beta_{1}} \\
& +\|\widetilde{W}\|_{\alpha, \beta_{1}}\left(1+\|\dot{Y}\|_{\infty}+\|\tilde{\widetilde{Y}}\|_{\infty}\right)^{\beta_{1}}\left\|\dot{Y}_{0}-\dot{\widetilde{Y}}\right\|_{V} \\
& +T^{\alpha}\left(1+\|\widetilde{W}\|_{\alpha, \beta_{1}}\right)\left(1+\|\dot{Y}\|_{\infty}+\|\dot{\widetilde{Y}}\|_{\infty}\right)^{\beta_{1}} d_{\alpha, W, \widetilde{W}}((Y, \dot{Y}),(\widetilde{Y}, \dot{\widetilde{Y}})) .
\end{aligned}
$$

Proof: Since $Y$ and $\widetilde{Y}$ are controlled by $W$ and $\widetilde{W}$ respectively, then we have

$$
\left\|Y_{s, t}-\widetilde{Y}_{s, t}\right\|_{V} \leq\left\|W_{s, t}\left(\dot{Y}_{s}\right)-\widetilde{W}_{s, t}\left(\dot{Y}_{s}\right)\right\|_{V}+\left\|\widetilde{W}_{s, t}\left(\dot{Y}_{s}\right)-\widetilde{W}_{s, t}\left(\dot{\widetilde{Y}}_{s}\right)\right\|_{V}+\left\|R_{s, t}^{Y}-R_{s, t}^{\widetilde{Y}}\right\|_{V} .
$$

Notice that by Taylor's Theorem 2.11,

$$
\begin{aligned}
\left\|\widetilde{W}_{s, t}\left(\dot{Y}_{s}\right)-\widetilde{W}_{s, t}\left(\dot{\widetilde{Y}}_{s}\right)\right\|_{V} & \leq \sup _{1 \leq \tau \leq 1}\left\|D \widetilde{W}_{s, t}\left(\tau \dot{Y}_{s}+(1-\tau) \dot{\tilde{Y}}_{s}\right)\left(\dot{Y}_{s}-\dot{\tilde{Y}}_{s}\right)\right\|_{V} \\
& \leq\|\widetilde{W}\|_{\alpha, \beta_{1}}\left(1+\|\dot{Y}\|_{\infty}+\|\dot{\widetilde{Y}}\|_{\infty}\right)^{\beta_{1}}\left\|\dot{Y}_{s}-\dot{\widetilde{Y}}_{s}\right\|_{V} .
\end{aligned}
$$

On the other hand, for any $Y \in \mathcal{C}^{\alpha}([0, T] ; V)$ we have

$$
\left\|Y_{s}\right\|_{V} \leq\left\|Y_{0}\right\|_{V}+\left\|Y_{s}-Y_{0}\right\|_{V} \leq\left\|Y_{0}\right\|_{V}+\|Y\|_{\alpha} s^{\alpha} .
$$


As a consequence, we can write

$$
\begin{aligned}
\left\|Y_{s, t}-\widetilde{Y}_{s, t}\right\|_{V} \leq & \|W-\widetilde{W}\|_{\alpha, \beta_{1}}\left(1+\|\dot{Y}\|_{\infty}\right)^{\beta_{0}}|t-s|^{\alpha} \\
& +\|\widetilde{W}\|_{\alpha, \beta_{1}}\left(1+\|\dot{Y}\|_{\infty}+\|\dot{\widetilde{Y}}\|_{\infty}\right)^{\beta_{1}}\left(\left\|\dot{Y}_{0}-\dot{\widetilde{Y}}_{0}\right\|_{V}+s^{\alpha}\|\dot{Y}-\dot{\widetilde{Y}}\|_{\alpha}\right)|t-s|^{\alpha} \\
& +\left\|R^{Y}-R^{\widetilde{Y}}\right\|_{2 \alpha}|t-s|^{2 \alpha} .
\end{aligned}
$$

This proves inequality (3.15).

Applying Lemma 3.7, the supremum norm of $Y-\widetilde{Y}$ can be estimated as follows:

$$
\begin{aligned}
\|Y-\widetilde{Y}\|_{\infty} & \leq\left\|Y_{0}-\widetilde{Y}_{0}\right\|_{V}+T^{\alpha}\|Y-\widetilde{Y}\|_{\alpha} \\
& \leq T^{\alpha}\left(1+\|\dot{Y}\|_{\infty}\right)^{\beta_{0}}\|W-\widetilde{W}\|_{\alpha, \beta_{3}} \\
& +\left(1+T^{\alpha}\right)\left(1+\|\widetilde{W}\|_{\alpha, \beta_{3}}\right)\left(1+\|\dot{Y}\|_{\infty}+\|\dot{\widetilde{Y}}\|_{\infty}\right)^{\beta_{1}}\left(\left\|Y_{0}-\widetilde{Y}_{0}\right\|_{V}+\left\|\dot{Y}_{0}-\dot{\widetilde{Y}}_{0}\right\|_{V}\right) \\
& +T^{2 \alpha}\left(1+\|\widetilde{W}\|_{\alpha, \beta_{3}}\right)\left(1+\|\dot{Y}\|_{\infty}+\|\dot{\tilde{Y}}\|_{\infty}\right)^{\beta_{1}} d_{\alpha, W, \widetilde{W}}((Y, \dot{Y}),(\widetilde{Y}, \dot{\tilde{Y}})) .
\end{aligned}
$$

Both inequalities (3.15) and (3.16) represent how the difference between $Y$ and $\tilde{Y}$ depends on $\|W-\widetilde{W}\|_{\alpha, \beta_{1}},\left\|\dot{Y}_{0}-\dot{\widetilde{Y}}_{0}\right\|_{V}$ and $d_{\alpha, W, \widetilde{W}}((Y, \dot{Y}),(\widetilde{Y}, \dot{\widetilde{Y}}))$. As we stated before, the factors $T^{\alpha}$ and $T^{2 \alpha}$ in each inequality are critical for the existence of the solution to equation (4.1) in Section 4.

Remark 3.8. $d_{\alpha, W, \widetilde{W}}$ defined in a subspace of $\mathcal{C}^{\alpha}([0, T] ; V) \times \mathcal{C}^{\alpha}([0, T] ; V)$ is not a metric, because the values of $Y_{0}, \widetilde{Y}_{0}$ or $\dot{Y}_{0}, \dot{\widetilde{Y}}_{0}$ may differ even if

$$
d_{\alpha, W, \widetilde{W}}((Y, \dot{Y}),(\widetilde{Y}, \dot{\tilde{Y}}))=0 .
$$

For any $\mathbf{y}=\left(y_{1}, y_{2}\right) \in V^{2}$, let

$$
\mathscr{E}_{W, \mathbf{y}}^{2 \alpha}=\left\{(Y, \dot{Y}) \in \mathscr{E}_{W}^{2 \alpha},\left(Y_{0}, \dot{Y}_{0}\right)=\left(y_{1}, y_{2}\right)\right\} .
$$

Then $d_{\alpha, W}=d_{\alpha, W, W}$ is really a metric on $\mathscr{E}_{W, \mathbf{y}}^{2 \alpha}$.

The next lemma shows that $\mathscr{E}_{W, \mathbf{y}}^{2 \alpha}$ is complete under the metric $d_{\alpha, W}$.

Lemma 3.9. Suppose that $W \in \mathcal{C}^{\alpha, \beta_{1}}([0, T] \times V ; V)$. Let $\mathbf{y}=\left(y_{1}, y_{2}\right) \in V^{2}$. Then $\left(\mathscr{E}_{W, \mathbf{y}}^{2 \alpha}, d_{\alpha, W}\right)$ is a complete metric space.

Proof: Suppose that $\left\{\left(Y^{n}, \dot{Y}^{n}\right)\right\}_{n \geq 1} \subset \mathscr{E}_{W, \mathbf{y}}^{2 \alpha}$ is a Cauchy sequence under the metric $d_{\alpha, W}$. We first show that $\left\{\left(Y^{n}, \dot{Y}^{n}, R^{Y^{n}}\right)\right\}_{n \geq 1}$ converges to $\left(Y, \dot{Y}, R^{Y}\right)$ in the product space $\mathcal{C}^{\alpha}([0, T] ; V) \times \mathcal{C}^{\alpha}([0, T] ; V) \times \mathcal{C}_{2}^{2 \alpha}\left([0, T]^{2} ; V\right)$ equipped with the Hölder seminorms. Notice that the space $\mathcal{C}^{\alpha}([0, T] ; V)$ is complete with the norm

$$
\|Y\|_{\mathcal{C}^{\alpha}([0, T] ; V)}:=\left\|Y_{0}\right\|_{V}+\|Y\|_{\alpha} .
$$

Thus there exists $\dot{Y} \in \mathcal{C}^{\alpha}([0, T] ; V)$, such that $\dot{Y}^{n} \rightarrow \dot{Y}$ as $n \rightarrow \infty$ pointwise and in $\mathcal{C}^{\alpha}([0, T] ; V)$. Next, we will show the convergence of $\left\{R^{Y^{n}}\right\}_{n \geq 1}$. Fix $(s, t) \in[0, T]^{2}$. Then, for and $n, m \geq 1$, we have

$$
\left\|R_{s, t}^{Y^{n}}-R_{s, t}^{Y^{m}}\right\|_{V} \leq|t-s|^{2 \alpha}\left\|R^{Y^{n}}-R^{Y^{m}}\right\|_{2 \alpha} .
$$

Therefore, $\left\{R_{s, t}^{Y^{n}}\right\}_{n \geq 1}$ is a Cauchy sequence in $V$, and thus has a limit denoted by $R_{s, t}^{Y}$. On the other hand, we can show that

$$
\limsup _{n \rightarrow \infty} \sup _{s \neq t \in[0, T]} \frac{\left\|R_{s, t}^{Y}-R_{s, t}^{Y^{n}}\right\|_{V}}{|t-s|^{2 \alpha}} \leq \lim _{n \rightarrow \infty} \lim _{m \rightarrow \infty} \sup _{s \neq t \in[0, T]} \frac{\left\|R_{s, t}^{Y^{m}}-R_{s, t}^{Y^{n}}\right\|_{V}}{|t-s|^{2 \alpha}}=0 .
$$


This implies that, as a sequence of functions, $\left\{R^{Y^{n}}\right\}_{n \geq 1}$ is also convergent in the space $\mathcal{C}_{2}^{2 \alpha}\left([0, T]^{2} ; V\right)$. To prove the convergence of $\left\{Y^{n}\right\}_{n \geq 1}$, it suffices to show that $\left\{Y^{n}\right\}_{n \geq 1}$ is Cauchy in $\mathcal{C}^{\alpha}([0, T] ; V)$ with the $\alpha$-Hölder seminorm. Notice that for any $n, m \geq 1, Y^{n}$ and $Y^{m}$ are both controlled by $W$, then, as a consequence of Lemma 3.7, we have

$$
\begin{aligned}
\left\|Y^{n}-Y^{m}\right\|_{\alpha} \leq & T^{\alpha}\left(1+\|W\|_{\alpha, \beta_{1}}\right)\left(1+\left\|\dot{Y}^{n}\right\|_{\infty}+\left\|\dot{Y}^{m}\right\|_{\infty}\right)^{\beta_{1}} \\
& \times d_{\alpha, W, \widetilde{W}}\left(\left(Y^{n}, \dot{Y}^{n}\right),\left(Y^{m}, \dot{Y}^{m}\right)\right) .
\end{aligned}
$$

Observe that

$$
\sup _{n \geq 1}\left\|\dot{Y}^{n}\right\|_{\infty} \leq y_{2}+T^{\alpha} \sup _{n \geq 1}\left\|\dot{Y}^{n}\right\|_{\alpha}=C<\infty .
$$

Therefore, $\left\{Y^{n}\right\}_{n \geq 1}$ converges to a function $Y$ in $\mathcal{C}^{\alpha}([0, T] ; V)$.

Finally, notice that for any $s, t \in[0, T]$,

$$
Y_{s, t}=\lim _{n \rightarrow \infty} Y_{s, t}^{n}=\lim _{n \rightarrow \infty}\left[W_{s, t}\left(\dot{Y}_{s}^{n}\right)+R_{s, t}^{Y^{n}}\right]=W_{s, t}\left(\dot{Y}_{s}\right)+R_{s, t}^{Y} .
$$

Thus $(Y, \dot{Y}) \in \mathscr{E}_{W, \mathbf{y}}^{2 \alpha}$ with the remainder $R^{Y}$.

In the next theorem, we define the nonlinear rough integral of a basic controlled rough path against a nonlinear rough path.

Theorem 3.10. Suppose that $\mathbf{W}=(W, \mathbb{W}) \in \mathscr{C}^{\alpha, \beta_{2}}([0, T] \times V ; V)$. Let $(Y, \dot{Y}) \epsilon$ $\mathscr{E}_{W}^{2 \alpha}$. We define $\Xi \in \mathcal{C}_{2}^{\alpha}\left([0, T]^{2} ; V\right)$ as follows:

$$
\Xi_{s, t}=W_{s, t}\left(Y_{s}\right)+\mathbb{W}_{s, t}\left(\dot{Y}_{s}, Y_{s}\right)
$$

Then the following limit exists

$$
\mathcal{J}_{s, t}(\Xi):=\lim _{|\pi| \rightarrow 0} \sum_{k=1}^{n} \Xi_{t_{k-1}, t_{k}}
$$

where $\pi=\left(s=t_{0}<t_{1}<\cdots<t_{n}=t\right)$. Moreover,

$$
\left\|\mathcal{J}_{s, t}(\Xi)-\Xi_{s, t}\right\|_{V} \leq C_{1}|t-s|^{3 \alpha}
$$

where

$C_{1}=k_{\alpha}\|\mathbf{W}\|_{\mathscr{C}_{2}}\left(1+2\|\dot{Y}\|_{\infty}\right)^{\beta_{0} \vee \beta_{1}}\left(1+2\|Y\|_{\infty}\right)^{\beta_{1} \vee \beta_{2}}\left(\|Y\|_{\alpha}+\|Y\|_{\alpha}^{2}+\|\dot{Y}\|_{\alpha}+\left\|R^{Y}\right\|_{2 \alpha}\right)$,

and $k_{\alpha}$ is defined in (2.5). ${ }^{1}$

Proof: For any $0 \leq s \leq u \leq t \leq T$, we write

$$
\begin{aligned}
\delta \Xi_{s, u, t} & =\Xi_{s, t}-\Xi_{s, u}-\Xi_{u, t} \\
& =-\left[W_{u, t}\left(Y_{u}\right)-W_{u, t}\left(Y_{s}\right)\right]+\left[\mathbb{W}_{s, t}\left(\dot{Y}_{s}, Y_{s}\right)-\mathbb{W}_{s, u}\left(\dot{Y}_{s}, Y_{s}\right)-\mathbb{W}_{u, t}\left(\dot{Y}_{u}, Y_{u}\right)\right] .
\end{aligned}
$$

According to Lemma 2.5, to prove (3.19) and (3.20), it suffices to show that $\left\|\delta \Xi_{s, u, t}\right\|_{V}$ is of order $O\left(|t-s|^{3 \alpha}\right)$. Recall notations (3.4) and (3.5). Since $Y$ is controlled by $W$, we can write

$$
\begin{aligned}
& W_{u, t}\left(Y_{u}\right)-W_{u, t}\left(Y_{s}\right)=D W_{u, t}\left(Y_{s}\right)\left(Y_{s, u}\right)+\mathscr{R}_{u, t}^{W}\left(Y_{s}, Y_{u}\right) \\
& =D W_{u, t}\left(Y_{s}\right)\left(W_{s, u}\left(\dot{Y}_{s}\right)\right)+D W_{u, t}\left(Y_{s}\right)\left(R_{s, u}^{Y}\right)+\mathscr{R}_{u, t}^{W}\left(Y_{s}, Y_{u}\right) .
\end{aligned}
$$

\footnotetext{
${ }^{1}$ We keep the track of the constituents of $C_{1}$ and all the constants appearing later for their use in the stability analysis of integrals and equations.
} 
On the other hand, by Chen's relation (3.10), we have

$$
\begin{aligned}
\mathbb{W}_{s, t}\left(\dot{Y}_{s}, Y_{s}\right)-\mathbb{W}_{s, u}\left(\dot{Y}_{s}, Y_{s}\right)-\mathbb{W}_{u, t}\left(\dot{Y}_{u}, Y_{u}\right) \\
=D W_{u, t}\left(Y_{s}\right)\left(W_{s, u}\left(\dot{Y}_{s}\right)\right)-\mathscr{D}_{u, t}^{\mathbb{W}}\left(\left(\dot{Y}_{s}, Y_{s}\right),\left(\dot{Y}_{u}, Y_{u}\right)\right) .
\end{aligned}
$$

Notice that, by definition, $\mathbb{W} \in \mathcal{C}_{2}^{2 \alpha, \beta_{1}^{\star}, \boldsymbol{\beta}_{1}^{* *}}\left([0, T]^{2} \times V^{2} ; V\right)$ where $\beta_{1}^{*}=\left(\beta_{0}, \beta_{0} \vee \beta_{1}\right)$ and $\beta_{1}^{* *}=\left(\beta_{1}, \beta_{1} \vee \beta_{2}\right)$. Combining (3.22) - (3.24), with (3.6) and (3.7) and recalling (3.11), we obtain the following inequality

$$
\begin{aligned}
& \left\|\delta \Xi_{s, u, t}\right\|_{V} \\
\leq & \left\|D W_{u, t}\left(Y_{s}\right)\right\|_{\mathfrak{L}_{1}(V ; V)}\left\|R_{s, u}^{Y}\right\|_{V}+\frac{1}{2}\|W\|_{\alpha, \boldsymbol{\beta}_{2}}\left(1+2\|Y\|_{\infty}\right)^{\beta_{2}}\|Y\|_{\alpha}^{2}|t-s|^{3 \alpha} \\
& +\|\mathbb{W}\|_{2 \alpha, \boldsymbol{\beta}_{1}^{*}, \boldsymbol{\beta}_{1}^{* *}}\left(1+2\|\dot{Y}\|_{\infty}\right)^{\beta_{1}^{*}}\left(1+2\|Y\|_{\infty}\right)^{\beta_{1}^{* *}}\left(\|Y\|_{\alpha}+\|\dot{Y}\|_{\alpha}\right)|t-s|^{3 \alpha} \\
\leq & \|\mathbf{W}\|_{\mathscr{C}_{2}}\left(1+2\|\dot{Y}\|_{\infty}\right)^{\beta_{0} \vee \beta_{1}}\left(1+2\|Y\|_{\infty}\right)^{\beta_{1} \vee \beta_{2}}\left(\|Y\|_{\alpha}+\|Y\|_{\alpha}^{2}+\|\dot{Y}\|_{\alpha}+\left\|R^{Y}\right\|_{2 \alpha}\right)|t-s|^{3 \alpha} .
\end{aligned}
$$

Thus we complete the proof by applying Lemma 2.5 .

Notice that $\mathcal{J}_{s, t}(\Xi)$ in Theorem 3.10 can be expressed as the limit of sums over a sequence of partitions $\pi^{n}$ as $\left|\pi^{n}\right| \rightarrow 0$. As a consequence of this fact, one can show that $\mathcal{J}_{s, t}(\Xi)$ is additive. Therefore, we can define the nonlinear integral of $Y$ against $W$ on any time interval $[s, t] \subset[0, T]$ by $\mathcal{J}_{s, t}(\Xi)$, that is

$$
\int_{s}^{t} W\left(d r, Y_{r}\right):=\mathcal{J}_{s, t}(\Xi) \text {. }
$$

By definition, we can easily verify that $\Xi$ in Theorem 3.10 is also $\alpha$-Hölder continuous. Recall that $\beta_{1}^{*}=\left(\beta_{0}, \beta_{0} \vee \beta_{1}\right)$ and $\beta_{1}^{\star *}=\left(\beta_{1}, \beta_{1} \vee \beta_{2}\right)$. Thus we have the following estimate,

$$
\begin{aligned}
\left\|\Xi_{s, t}\right\|_{V} \leq & \left\|W_{s, t}\left(Y_{s}\right)\right\|_{V}+\left\|\mathbb{W}_{s, t}\left(\dot{Y}_{s}, Y_{s}\right)\right\|_{V} \\
& \leq\|W\|_{\alpha, \beta_{2}}\left(1+\|Y\|_{\infty}\right)^{\beta_{0}}|t-s|^{\alpha} \\
& +\|\mathbb{W}\|_{2 \alpha, \beta_{1}^{*}, \boldsymbol{\beta}_{1}^{* *}}\left(1+\|\dot{Y}\|_{\infty}\right)^{\beta_{0}}\left(1+\|Y\|_{\infty}\right)^{\beta_{1}}|t-s|^{2 \alpha}
\end{aligned}
$$

The following estimates follow from (3.20) and (3.27):

$$
\begin{aligned}
\left\|\int_{s}^{t} W\left(d r, Y_{r}\right)\right\|_{V} & \leq\left\|\Xi_{s, t}\right\|_{V}+\left\|\mathcal{J}_{s, t}(\Xi)-\Xi_{s, t}\right\|_{V} \\
& \leq C_{2}|t-s|^{\alpha},
\end{aligned}
$$

where

$$
C_{2}=C_{1} T^{2 \alpha}+\|W\|_{\alpha, \boldsymbol{\beta}_{2}}\left(1+\|Y\|_{\infty}\right)^{\beta_{0}}+T^{\alpha}\|\mathbb{W}\|_{2 \alpha, \boldsymbol{\beta}_{1}^{*}, \boldsymbol{\beta}_{1}^{* *}}\left(1+\|\dot{Y}\|_{\infty}\right)^{\beta_{0}}\left(1+\|Y\|_{\infty}\right)^{\beta_{1}} .
$$

Remark 3.11. To define a nonlinear rough integral, the growth condition on $(W, \mathbb{W})$ is not necessary. In fact, let $\mathscr{C}_{l o c}^{\alpha, 2}([0, T] \times V ; V)$ be the collection of pairs $(W, \mathbb{W})$ such that $W:[0, T] \times V \rightarrow V$ is $\alpha$-Hölder in time, and twice differentiable in space with locally bounded spatial derivatives, $\mathbb{W}:[0, T]^{2} \times V \rightarrow V$ is $2 \alpha$-Hölder continuous in time, and differentiable in space with locally bounded spatial derivative, and Chen's relation (3.10) holds. For any $\mathbf{W}=(W, \mathbb{W}) \in \mathscr{C}_{l o c}^{\alpha, 2}([0, T] \times V ; V)$, and $(Y, \dot{Y}) \in \mathscr{E}_{W}^{2 \alpha}$, the expression (3.19) is still a well-defined nonlinear rough integral. However, the growth condition is really needed to consider the global existence of RDEs (see Section 4.2). 
3.2. Properties of nonlinear rough integrals. In this section, we present some properties of nonlinear rough integrals. The next proposition shows that the nonlinear rough integral is a basic nonlinear controlled rough path (see Proposition 2.6 for the linear result).

Proposition 3.12. Let $\mathbf{W}=(W, \mathbb{W}) \in \mathscr{C}^{\alpha, \beta_{2}}([0, T] \times V ; V)$. Suppose that $(Y, \dot{Y}) \epsilon$ $\mathscr{E}_{W}^{2 \alpha}$. Let $Z:[0, T] \rightarrow V$ be the nonlinear rough integral of $Y$ against $W$ in the sense of (3.26):

$$
Z_{t}=\int_{0}^{t} W\left(d r, Y_{r}\right)
$$

Then, $Z$ is controlled by $W:(Z, \dot{Z})=(Z, Y) \in \mathscr{E}_{W}^{2 \alpha}$.

Proof: Let $R_{s, t}^{Z}:=Z_{s, t}-W_{s, t}\left(Y_{s}\right)$. Then by (3.20), we can write

$$
\begin{aligned}
& \left\|R_{s, t}^{Z}\right\|_{V}=\left\|\int_{s}^{t} W\left(d r, Y_{r}\right)-W_{s, t}\left(Y_{s}\right)\right\|_{V} \\
& \quad \leq\left\|\mathcal{J}_{s, t}(\Xi)-\Xi_{s, t}\right\|_{V}+\left\|\mathbb{W}_{s, t}\left(\dot{Y}_{s}, Y_{s}\right)\right\|_{V} \\
& \quad \leq C_{1}|t-s|^{3 \alpha}+\|\mathbb{W}\|_{2 \alpha, \boldsymbol{\beta}_{2}^{*}, \boldsymbol{\beta}_{2}^{* *}}\left(1+\|\dot{Y}\|_{\infty}\right)^{\beta_{0}}\left(1+\|Y\|_{\infty}\right)^{\beta_{1}}|t-s|^{2 \alpha},
\end{aligned}
$$

where $C_{1}$ is the constant appearing in (3.21). It follows that

$$
\begin{aligned}
\left\|R^{Z}\right\|_{2 \alpha} \leq & k_{\alpha}\|\mathbf{W}\|_{\mathscr{C}}\left(1+2\|\dot{Y}\|_{\infty}\right)^{\beta_{0} \vee \beta_{1}}\left(1+2\|Y\|_{\infty}\right)^{\beta_{1} \vee \beta_{2}} \\
& \times\left[1+T^{\alpha}\left(\|Y\|_{\alpha}+\|Y\|_{\alpha}^{2}+\|\dot{Y}\|_{\alpha}+\left\|R^{Y}\right\|_{2 \alpha}\right)\right] .
\end{aligned}
$$

As a consequence, $Z$ is controlled by $W$ with the Gubinelli derivative $\dot{Z}=Y$.

In the next proposition, we consider the stability of nonlinear rough integrals.

Proposition 3.13. Let $\mathbf{W}, \widetilde{\mathbf{W}} \in \mathscr{C}^{\alpha, \boldsymbol{\beta}_{3}}([0, T] \times V ; V)$. Suppose that $(Y, \dot{Y}) \in \mathscr{E}_{W}^{2 \alpha}$ and $(\widetilde{Y}, \dot{\widetilde{Y}}) \in \mathscr{E} \frac{2 \alpha}{W}$. Define

$$
Z_{t}=\int_{0}^{t} W\left(d r, Y_{r}\right) \text { and } \widetilde{Z}_{t}=\int_{0}^{t} \widetilde{W}\left(d r, \widetilde{Y}_{r}\right) .
$$

Then $(Z, Y) \in \mathscr{E}_{W}^{2 \alpha}$ and $(\widetilde{Z}, \widetilde{Y}) \in \mathscr{E}_{\frac{W}{W}}^{2 \alpha}$ by Proposition 3.12. In addition, the following inequality holds:

$$
\begin{aligned}
d_{\alpha, W, \widetilde{W}}((Z, Y),(\widetilde{Z}, \widetilde{Y})) \leq & C_{3} \varrho_{\alpha, \beta_{3}}(\mathbf{W}, \widetilde{\mathbf{W}})+C_{4}\left(\left\|Y_{0}-\widetilde{Y}_{0}\right\|_{V}+\left\|\dot{Y}_{0}-\dot{\tilde{Y}}_{0}\right\|_{V}\right) \\
& +C_{5} d_{\alpha, W, \widetilde{W}}((Y, \dot{Y}),(\widetilde{Y}, \dot{\tilde{Y}})),
\end{aligned}
$$

where

$$
\begin{aligned}
C_{3}=2 k_{\alpha}\left(1+T^{\alpha}\right)^{2}\left(1+\|\widetilde{\mathbf{W}}\|_{\mathscr{C}_{3}}\right)\left(1+2\|Y\|_{\infty}+2\|\widetilde{Y}\|_{\infty}\right)^{\beta_{2}^{* *}}\left(1+2\|\dot{Y}\|_{\infty}+2\|\dot{\widetilde{Y}}\|_{\infty}\right)^{\beta_{2}^{*}+\beta_{0} \vee \beta_{1}} \\
\times\left[1+\|Y\|_{\alpha}+\|\dot{Y}\|_{\alpha}+\|\widetilde{Y}\|_{\alpha}+\|\dot{\widetilde{Y}}\|_{\alpha}+\left(\|Y\|_{\alpha}+\|\widetilde{Y}\|_{\alpha}\right)^{2}+\left\|R^{Y}\right\|_{2 \alpha}\right], \\
C_{4}=5 k_{\alpha}\left(1+T^{\alpha}\right)^{2}\left(\|\widetilde{\mathbf{W}}\|_{\mathscr{C}_{3}}+\|\widetilde{\mathbf{W}}\|_{\mathscr{C}_{3}}^{2}\right)\left(1+2\|Y\|_{\infty}+2\|\widetilde{Y}\|_{\infty}\right)^{\beta_{2}^{* *}}\left(1+2\|\dot{Y}\|_{\infty}+2\|\dot{\widetilde{Y}}\|_{\infty}\right)^{\beta_{2}^{*}+\beta_{1}} \\
\times\left[1+\|Y\|_{\alpha}+\|\dot{Y}\|_{\alpha}+\|\widetilde{Y}\|_{\alpha}+\|\dot{\widetilde{Y}}\|_{\alpha}+\left(\|Y\|_{\alpha}+\|\widetilde{Y}\|_{\alpha}\right)^{2}+\left\|R^{Y}\right\|_{2 \alpha}\right] \\
C_{5}=6 k_{\alpha} T^{\alpha}\left(1+T^{\alpha}\right)\left(1+\|\widetilde{\mathbf{W}}\|_{\mathscr{C}_{3}}\right)^{2}\left(1+2\|Y\|_{\infty}+2\|\widetilde{Y}\|_{\infty}\right)^{\beta_{2}^{* *}}\left(1+2\|\dot{Y}\|_{\infty}+2\|\dot{\tilde{Y}}\|_{\infty}\right)^{\beta_{2}^{*}+\beta_{1}} \\
\times\left[1+T^{\alpha}\left(\|Y\|_{\alpha}+\|\dot{Y}\|_{\alpha}+\|\widetilde{Y}\|_{\alpha}+\|\dot{\widetilde{Y}}\|_{\alpha}\right)+T^{2 \alpha}\left(\|Y\|_{\alpha}+\|\widetilde{Y}\|_{\alpha}\right)^{2}+T^{2 \alpha}\left\|R^{Y}\right\|_{2 \alpha}\right], \\
\beta_{2}^{*}=\max \left\{\beta_{0}, \beta_{1}, \beta_{2}\right\} \text { and } \beta_{2}^{* *}=\max \left\{\beta_{1}, \beta_{2}, \beta_{3}\right\} .
\end{aligned}
$$


Proof: Due to Lemma 3.7, it suffices to estimate $\left\|R^{Z}-R^{\widetilde{Z}}\right\|_{2 \alpha}$. Let $\Xi$ and $\widetilde{\Xi}$ be the approximations of $Z$ and $\widetilde{Z}$ respectively. That is,

$$
\Xi_{s, t}=W_{s, t}\left(Y_{s}\right)+\mathbb{W}_{s, t}\left(\dot{Y}_{s}, Y_{s}\right) \quad \text { and } \quad \widetilde{\Xi}_{s, t}=\widetilde{W}_{s, t}\left(\widetilde{Y}_{s}\right)+\widetilde{W}_{s, t}\left(\dot{\tilde{Y}}_{s}, \widetilde{Y}_{s}\right)
$$

Set $\Delta=\Xi-\widetilde{\Xi}$. Then by Proposition 3.12, we know that

$$
\left\|R_{s, t}^{Z}-R_{s, t}^{\widetilde{Z}}\right\|_{V} \leq\left\|Z_{s, t}-\widetilde{Z}_{s, t}-\Delta_{s, t}\right\|_{V}+\left\|\mathbb{W}_{s, t}\left(\dot{Y}_{s}, Y_{s}\right)-\widetilde{\mathbb{W}}_{s, t}\left(\dot{\bar{Y}}_{s}, \widetilde{Y}_{s}\right)\right\|_{V} .
$$

Due to the Sewing Lemma 2.5, to estimate the first term on the right-hand side of (3.32), it suffices to estimate $\|\delta \Delta\|_{V}$. Taking into account formulas (3.22) - (3.24), we can write

$$
\begin{aligned}
-\delta \Delta_{s, u, t}= & {\left[D W_{u, t}\left(Y_{s}\right)\left(R_{s, u}^{Y}\right)-D \widetilde{W}_{u, t}\left(\widetilde{Y}_{s}\right)\left(R_{s, u}^{\widetilde{Y}}\right)\right]+\left[\mathscr{R}_{u, t}^{W}\left(Y_{s}, Y_{u}\right)-\mathscr{R}_{u, t}^{\widetilde{W}}\left(\widetilde{Y}_{s}, \widetilde{Y}_{u}\right)\right] } \\
& +\left[\mathscr{D}_{u, t}^{\mathbb{W}}\left(\left(\dot{Y}_{s}, Y_{s}\right),\left(\dot{Y}_{u}, Y_{u}\right)\right)-\mathscr{D}_{u, t}^{\widetilde{W}}\left(\left(\dot{\widetilde{Y}}_{s}, \widetilde{Y}_{s}\right),\left(\dot{\widetilde{Y}}_{u}, \widetilde{Y}_{u}\right)\right)\right] \\
:= & J_{1}+J_{2}+J_{3},
\end{aligned}
$$

where $\mathscr{R}^{W}, \mathscr{R}^{\widetilde{W}}, \mathscr{D}^{W}$ and $\mathscr{D}^{\widetilde{W}}$ are defined as in (3.4) and (3.5), respectively.

Estimates for $J_{1}$ : Triangular inequality implies that

$$
\begin{aligned}
\left\|J_{1}\right\|_{V} \leq & \left\|D W_{u, t}\left(Y_{s}\right)\left(R_{s, u}^{Y}\right)-D \widetilde{W}_{u, t}\left(Y_{s}\right)\left(R_{s, u}^{Y}\right)\right\|_{V} \\
& +\left\|D \widetilde{W}_{u, t}\left(Y_{s}\right)\left(R_{s, u}^{Y}\right)-D \widetilde{W}_{u, t}\left(\widetilde{Y}_{s}\right)\left(R_{s, u}^{Y}\right)\right\|_{V} \\
& +\left\|D \widetilde{W}_{u, t}\left(\widetilde{Y}_{s}\right)\left(R_{s, u}^{Y}\right)-D \widetilde{W}_{u, t}\left(\widetilde{Y}_{s}\right)\left(R_{s, u}^{\widetilde{Y}}\right)\right\|_{V} \\
\leq & \|W-\widetilde{W}\|_{\alpha, \beta_{3}}\left(1+\|Y\|_{\infty}\right)^{\beta_{1}}\left\|R^{Y}\right\|_{2 \alpha}|t-s|^{3 \alpha} \\
& +\|\widetilde{W}\|_{\alpha, \beta_{3}}\left(1+\|Y\|_{\infty}+\|\widetilde{Y}\|_{\infty}\right)^{\beta_{2}}\left\|Y_{s}-\widetilde{Y}_{s}\right\|_{V}\left\|R^{Y}\right\|_{2 \alpha}|t-s|^{3 \alpha} \\
& +\|\widetilde{W}\|_{\alpha, \beta_{3}}\left(1+\|\widetilde{Y}\|_{\infty}\right)^{\beta_{1}}\left\|R^{Y}-R^{\widetilde{Y}}\right\|_{2 \alpha}|t-s|^{3 \alpha} .
\end{aligned}
$$

Plugging (3.16) into (3.34), we have

$$
\begin{aligned}
\left\|J_{1}\right\|_{V} \leq & \left\{\left(1+T^{\alpha}\right)\left(1+\|\widetilde{W}\|_{\alpha, \beta_{3}}\right)\left(1+\|Y\|_{\infty}+\|\widetilde{Y}\|_{\infty}\right)^{\beta_{1} \vee \beta_{2}}\left(1+\|\dot{Y}\|_{\infty}\right)^{\beta_{0}}\right. \\
& \times\left\|R^{Y}\right\|_{2 \alpha}\|W-\widetilde{W}\|_{\alpha, \beta_{3}} \\
+ & \left(1+T^{\alpha}\right)\left(\|\widetilde{W}\|_{\alpha, \beta_{3}}+\|\widetilde{W}\|_{\alpha, \beta_{3}}^{2}\right)\left(1+\|Y\|_{\infty}+\|\widetilde{Y}\|_{\infty}\right)^{\beta_{1} \vee \beta_{2}} \\
& \times\left(1+\|\dot{Y}\|_{\infty}+\|\dot{\widetilde{Y}}\|_{\infty}\right)^{\beta_{1}}\left\|R^{Y}\right\|_{2 \alpha}\left(\left\|Y_{0}-\widetilde{Y}_{0}\right\|_{V}+\left\|\dot{Y}_{0}-\dot{\widetilde{Y}}\right\|_{V}\right) \\
+ & \left(\|\widetilde{W}\|_{\alpha, \beta_{3}}+\|\widetilde{W}\|_{\alpha, \beta_{3}}^{2}\right)\left(1+\|Y\|_{\infty}+\|\widetilde{Y}\|_{\infty}\right)^{\beta_{1} \vee \beta_{2}}\left(1+\|\dot{Y}\|_{\infty}+\|\dot{\widetilde{Y}}\|_{\infty}\right)^{\beta_{1}} \\
& \left.\times\left(1+T^{2 \alpha}\left\|R^{Y}\right\|_{2 \alpha}\right) d_{\alpha, W, \widetilde{W}}((Y, \dot{Y}),(\widetilde{Y}, \dot{\widetilde{Y}}))\right\}|t-s|^{3 \alpha}
\end{aligned}
$$

Estimates for $J_{2}$ : In order to bound $J_{2}$, we decompose $J_{2}$ as follows

$$
J_{2}=\mathscr{R}_{u, t}^{W-\widetilde{W}}\left(Y_{s}, Y_{u}\right)+\left[\mathscr{R}_{u, t}^{\widetilde{W}}\left(Y_{s}, Y_{u}\right)-\mathscr{R}_{u, t}^{\widetilde{W}}\left(\widetilde{Y}_{s}, \widetilde{Y}_{u}\right)\right]:=J_{2}^{1}+J_{2}^{2} .
$$

By (3.6), we can write

$$
\left\|J_{2}^{1}\right\|_{V} \leq \frac{1}{2}\left(1+2\|Y\|_{\infty}\right)^{\beta_{2}}\|Y\|_{\alpha}^{2}\|W-\widetilde{W}\|_{\alpha, \beta_{3}}|t-s|^{3 \alpha} .
$$


Thus, using Taylor's Theorem 2.11 and inequality (3.8), we have

$$
\begin{aligned}
\left\|J_{2}^{2}\right\|_{V} & \leq \sup _{\tau \in[0,1]}\left\|D \mathscr{R}_{u, t}^{\widetilde{W}}\left(\tau Y_{s}+(1-\tau) \widetilde{Y}_{s}, \tau Y_{u}+(1-\tau) \widetilde{Y}_{u}\right)\left(Y_{s}-\widetilde{Y}_{s}, Y_{u}-\widetilde{Y}_{u}\right)\right\|_{V} \\
& \leq|t-s|^{\alpha}\|\widetilde{W}\|_{\alpha, \beta_{3}}\left(1+2\|Y\|_{\infty}+2\|\widetilde{Y}\|_{\infty}\right)^{\beta_{2} \vee \beta_{3}} \\
& \times\left[\left\|\tau Y_{s, u}+(1-\tau) \widetilde{Y}_{s, u}\right\|_{V}^{2}\left\|Y_{u}-\widetilde{Y}_{u}\right\|_{V}+\left\|\tau Y_{s, u}+(1-\tau) \widetilde{Y}_{s, u}\right\| V\left\|(Y-\widetilde{Y})_{s, u}\right\|_{V}\right] \\
& \leq\|\widetilde{W}\|_{\alpha, \beta_{3}}\left(1+2\|Y\|_{\infty}+2\|\widetilde{Y}\|_{\infty}\right)^{\beta_{2} \vee \beta_{3}} \\
& \times\left[b\left(\|Y\|_{\alpha}+\|\widetilde{Y}\|_{\alpha}\right)^{2}\|Y-\widetilde{Y}\|_{\infty}+\left(\|Y\|_{\alpha}+\|\widetilde{Y}\|_{\alpha}\right)\|Y-\widetilde{Y}\|_{\alpha}\right]|t-s|^{3 \alpha} .
\end{aligned}
$$

Applying (3.15) and (3.16), and putting together the terms with $\|W-\widetilde{W}\|_{\alpha, \beta_{3}}$, $\left(\left\|Y_{0}-\widetilde{Y}_{0}\right\|_{V}+\left\|\dot{Y}_{0}-\dot{\tilde{Y}}_{0}\right\|_{V}\right)$ and $d_{\alpha, W, \widetilde{W}}((Y, \dot{Y}),(\widetilde{Y}, \dot{\tilde{Y}}))$, respectively, we have

$$
\begin{aligned}
\left\|J_{2}^{2}\right\|_{V} \leq & {\left[F_{1} \times\|W-\widetilde{W}\|_{\alpha, \beta_{3}}+F_{2} \times\left(\left\|Y_{0}-\widetilde{Y}_{0}\right\|_{V}+\left\|\dot{Y}_{0}-\dot{\tilde{Y}}_{0}\right\|_{V}\right)\right.} \\
& \left.+F_{3} \times d_{\alpha, W, \widetilde{W}}((Y, \dot{Y}),(\widetilde{Y}, \dot{\widetilde{Y}}))\right]|t-s|^{3 \alpha}
\end{aligned}
$$

where

$$
\begin{gathered}
F_{1}=\|\widetilde{W}\|_{\alpha, \beta_{3}}\left(1+2\|Y\|_{\infty}+2\|\widetilde{Y}\|_{\infty}\right)^{\beta_{2} \vee \beta_{3}}\left(1+\|\dot{Y}\|_{\infty}\right)^{\beta_{0}} \\
\times\left(\left(\|Y\|_{\alpha}+\|\widetilde{Y}\|_{\alpha}\right)+T^{\alpha}\left(\|Y\|_{\alpha}+\|\widetilde{Y}\|_{\alpha}\right)^{2}\right), \\
F_{2}=\left(1+T^{\alpha}\right)\left(\|\widetilde{W}\|_{\alpha, \beta_{3}}+\|\widetilde{W}\|_{\alpha, \beta_{3}}^{2}\right)\left(1+2\|Y\|_{\infty}+2\|\widetilde{Y}\|_{\infty}\right)^{\beta_{2} \vee \beta_{3}}\left(1+\|\dot{Y}\|_{\infty}+\|\dot{\widetilde{Y}}\|_{\infty}\right)^{\beta_{1}} \\
\times\left(\left(\|Y\|_{\alpha}+\|\widetilde{Y}\|_{\alpha}\right)+\left(\|Y\|_{\alpha}+\|\widetilde{Y}\|_{\alpha}\right)^{2}\right)
\end{gathered}
$$

and

$$
\begin{aligned}
F_{3}= & \left(\|\widetilde{W}\|_{\alpha, \beta_{3}}+\|\widetilde{W}\|_{\alpha, \beta_{3}}^{2}\right)\left(1+2\|Y\|_{\infty}+2\|\widetilde{Y}\|_{\infty}\right)^{\beta_{2} \vee \beta_{3}}\left(1+\|\dot{Y}\|_{\infty}+\|\dot{\tilde{Y}}\|_{\infty}\right)^{\beta_{1}} \\
& \times\left(T^{\alpha}\left(\|Y\|_{\alpha}+\|\widetilde{Y}\|_{\alpha}\right)+T^{2 \alpha}\left(\|Y\|_{\alpha}+\|\widetilde{Y}\|_{\alpha}\right)^{2}\right) .
\end{aligned}
$$

Estimates for $J_{3}$ : Similarly, we decompose $J_{3}$ as follows

$$
\begin{aligned}
J_{3} & =\mathscr{D}_{u, t}^{\mathbb{W}-\widetilde{\mathbb{W}}}\left(\left(\dot{Y}_{s}, Y_{s}\right),\left(\dot{Y}_{u}, Y_{u}\right)\right)+\left[\mathscr{D}_{u, t}^{\widetilde{\mathbb{W}}}\left(\left(\dot{Y}_{s}, Y_{s}\right),\left(\dot{Y}_{u}, Y_{u}\right)\right)-\mathscr{D}_{u, t}^{\widetilde{\mathbb{W}}}\left(\left(\dot{\tilde{Y}}_{s}, \widetilde{Y}_{s}\right),\left(\dot{\tilde{Y}}_{u}, \widetilde{Y}_{u}\right)\right)\right] \\
& :=J_{3}^{1}+J_{3}^{2} .
\end{aligned}
$$

The estimate for $J_{3}^{1}$ can by obtained by inequality (3.7), that is

$$
\begin{aligned}
\left\|J_{3}^{1}\right\|_{V} \leq & \left(1+2\|\dot{Y}\|_{\infty}\right)^{\beta_{0} \vee \beta_{1}}\left(1+2\|Y\|_{\infty}\right)^{\beta_{1} \vee \beta_{2}}\left(\|\dot{Y}\|_{\alpha}+\|Y\|_{\alpha}\right) \\
& \times\|\mathbb{W}-\widetilde{\mathbb{W}}\|_{2 \alpha, \boldsymbol{\beta}_{2}^{*}, \boldsymbol{\beta}_{2}^{* *}}|t-s|^{3 \alpha} .
\end{aligned}
$$

To bound $J_{3}^{2}$, we apply Taylor's Theorem 2.11 and get

$$
\left\|J_{3}^{2}\right\|_{V} \leq \sup _{0 \leq \tau \leq 1}\left\|D \mathscr{D}_{u, t}^{\widetilde{\mathbb{W}}}(\xi(\tau))\left(\dot{Y}_{s}-\dot{\tilde{Y}}_{s}, Y_{s}-\widetilde{Y}_{s}, \dot{Y}_{u}-\dot{\tilde{Y}}_{u}, Y_{u}-\widetilde{Y}_{u}\right)\right\|_{V},
$$


where $\boldsymbol{\xi}(\tau)=\tau\left(\dot{Y}_{s}, Y_{s}, \dot{Y}_{u}, Y_{u}\right)+(1-\tau)\left(\dot{\widetilde{Y}}_{s}, \widetilde{Y}_{s}, \dot{\widetilde{Y}}_{u}, \widetilde{Y}_{u}\right)$. Therefore, using inequalities (3.9), (3.15) and (3.16), we can show that

$$
\begin{aligned}
\left\|J_{3}^{2}\right\|_{V} \leq & \left(1+T^{\alpha}\right)\|\widetilde{\mathbf{W}}\|_{2 \alpha, \beta_{2}^{*}, \beta_{2}^{* *}}\left(1+2\|Y\|_{\infty}+2\|\widetilde{Y}\|_{\infty}\right)^{\beta_{2}^{* *}}\left(1+2\|\dot{Y}\|_{\infty}+2\|\dot{\widetilde{Y}}\|_{\infty}\right)^{\beta_{2}^{*}+\beta_{0}} \\
& \times\left(\|Y\|_{\alpha}+\|\dot{Y}\|_{\alpha}+\|\widetilde{Y}\|_{\alpha}+\|\dot{\widetilde{Y}}\|_{\alpha}\right)\|W-\widetilde{W}\|_{\alpha, \beta_{3}} \\
+ & 3\left(1+T^{\alpha}\right)\left(\|\widetilde{\mathbf{W}}\|_{\mathscr{C}_{3}}+\|\widetilde{\mathbf{W}}\|_{\mathscr{C}_{3}}^{2}\right)\left(\|Y\|_{\alpha}+\|\dot{Y}\|_{\alpha}+\|\widetilde{Y}\|_{\alpha}+\|\dot{\widetilde{Y}}\|_{\alpha}\right) \\
& \times\left(1+2\|Y\|_{\infty}+2\|\widetilde{Y}\|_{\infty}\right)^{\beta_{2}^{* *}}\left(1+2\|\dot{Y}\|_{\infty}+2\|\dot{\widetilde{Y}}\|_{\infty}\right)^{\beta_{2}^{*}+\beta_{1}} \\
& \times\left(\left\|Y_{0}-\widetilde{Y}\right\|_{V}+\left\|\dot{Y}_{0}-\dot{\widetilde{Y}}\right\|_{V}\right) \\
+ & 2\left(1+T^{\alpha}\right)\left(\|\widetilde{\mathbf{W}}\|_{\mathscr{C}_{3}}+\|\widetilde{\mathbf{W}}\|_{\mathscr{C}_{3}}^{2}\right)\left(1+T^{\alpha}\left(\|Y\|_{\alpha}+\|\dot{Y}\|_{\alpha}+\|\widetilde{Y}\|_{\alpha}+\|\dot{\widetilde{Y}}\|_{\alpha}\right)\right) \\
& \times\left(1+2\|Y\|_{\infty}+2\|\widetilde{Y}\|_{\infty}\right)^{\beta_{2}^{* *}}\left(1+2\|\dot{Y}\|_{\infty}+2\|\dot{\widetilde{Y}}\|_{\infty}\right)^{\beta_{2}^{*}+\beta_{1}} \\
& \left.\times d_{\alpha, W, \widetilde{W}}((Y, \dot{Y}),(\widetilde{Y}, \dot{\widetilde{Y}}))\right]|t-s|^{3 \alpha}
\end{aligned}
$$

Therefore, combining (3.33) and (3.35) - (3.39), we have

$$
\begin{aligned}
&\left\|\delta \Delta_{s, u, t}\right\|_{V} \\
& \leq\{\left(1+T^{\alpha}\right)\left(1+\|\widetilde{\mathbf{W}}\|_{\mathscr{C}_{3}}\right)\left(1+2\|Y\|_{\infty}+2\|\widetilde{Y}\|_{\infty}\right)^{\beta_{2}^{* *}}\left(1+2\|\dot{Y}\|_{\infty}+2\|\dot{\widetilde{Y}}\|_{\infty}\right)^{\beta_{2}^{*}+\beta_{0}} \\
& \times\left[\|Y\|_{\alpha}+\|\dot{Y}\|_{\alpha}+\|\widetilde{Y}\|_{\alpha}+\|\dot{\widetilde{Y}}\|_{\alpha}+\left(\|Y\|_{\alpha}+\|\widetilde{Y}\|_{\alpha}\right)^{2}+\left\|R^{Y}\right\|_{2 \alpha}\right] \varrho_{\alpha, \beta_{3}}(\mathbf{W}, \widetilde{\mathbf{W}}) \\
&+ 4\left(1+T^{\alpha}\right)\left(\|\widetilde{\mathbf{W}}\|_{\mathscr{C}_{3}}+\|\widetilde{\mathbf{W}}\|_{\mathscr{C}_{3}}^{2}\right)\left(1+2\|Y\|_{\infty}+2\|\widetilde{Y}\|_{\infty}\right)^{\beta_{2}^{* *}}\left(1+2\|\dot{Y}\|_{\infty}+2\|\dot{\widetilde{Y}}\|_{\infty}\right)^{\beta_{2}^{*}+\beta_{1}} \\
& \times\left[\|Y\|_{\alpha}+\|\dot{Y}\|_{\alpha}+\|\widetilde{Y}\|_{\alpha}+\|\dot{\widetilde{Y}}\|_{\alpha}+\left(\|Y\|_{\alpha}+\|\widetilde{Y}\|_{\alpha}\right)^{2}+\left\|R^{Y}\right\|_{2 \alpha}\right] \\
& \times\left(\left\|Y_{0}-\widetilde{Y}\right\|_{V}+\left\|\dot{Y_{0}}-\dot{\widetilde{Y}}\right\|_{V}\right) \\
&+ 4\left(1+T^{\alpha}\right)\left(\|\widetilde{\mathbf{W}}\|_{\mathscr{C}_{3}}+\|\widetilde{\mathbf{W}}\|_{\mathscr{C}_{3}}^{2}\right)\left(1+2\|Y\|_{\infty}+2\|\widetilde{Y}\|_{\infty}\right)^{\beta_{2}^{* *}}\left(1+2\|\dot{Y}\|_{\infty}+2\|\dot{\widetilde{Y}}\|_{\infty}\right)^{\beta_{2}^{*}+\beta_{1}} \\
& \times\left[1+T^{\alpha}\left(\|Y\|_{\alpha}+\|\dot{Y}\|_{\alpha}+\|\widetilde{Y}\|_{\alpha}+\|\dot{\widetilde{Y}}\|_{\alpha}\right)+T^{2 \alpha}\left(\|Y\|_{\alpha}+\|\widetilde{Y}\|_{\alpha}\right)^{2}+T^{2 \alpha}\left\|R^{Y}\right\|_{2 \alpha}\right] \\
&\left.\times d_{\alpha, W, \widetilde{W}}((Y, \dot{Y}),(\widetilde{Y}, \dot{\tilde{Y}}))\right\}|t-s|^{3 \alpha} .
\end{aligned}
$$

On the other hand, by (3.7) and (3.16), we can show that

$$
\begin{aligned}
&\left\|\mathbb{W}_{s, t}\left(\dot{Y}_{s}, Y_{s}\right)-\widetilde{\mathbb{W}}_{s, t}\left(\dot{\widetilde{Y}}_{s}, \widetilde{Y}_{s}\right)\right\|_{V}=\left\|(\mathbb{W}-\widetilde{\mathbb{W}})_{s, t}\left(\dot{Y}_{s}, Y_{s}\right)-\mathscr{D}_{s, t}^{\widetilde{W}_{1}}\left(\left(\dot{Y}_{s}, Y_{s}\right),\left(\dot{\widetilde{Y}}_{s}, \widetilde{Y}_{s}\right)\right)\right\|_{V} \\
& \leq\{\left(1+T^{\alpha}\right)\left(1+\|\widetilde{\mathbf{W}}\|_{\mathscr{C}_{3}}\right)\left(1+\|\dot{Y}\|_{\infty}+\|\dot{\widetilde{Y}}\|_{\infty}\right)^{2 \beta_{0} \vee \beta_{1}}\left(1+\|Y\|_{\infty}+\|\widetilde{Y}\|_{\infty}\right)^{\beta_{1} \vee \beta_{2}} \\
& \times \varrho_{\alpha, \beta_{3}}(\mathbf{W}, \widetilde{\mathbf{W}}) \\
&+ 2\left(1+T^{\alpha}\right)\left(\|\widetilde{\mathbf{W}}\|_{\mathscr{C}_{3}}+\|\widetilde{\mathbf{W}}\|_{\mathscr{C}_{3}}^{2}\right)\left(1+\|\dot{Y}\|_{\infty}+\|\dot{\widetilde{Y}}\|_{\infty}\right)^{\beta_{0} \vee \beta_{1}+\beta_{1}}\left(1+\|Y\|_{\infty}+\|\widetilde{Y}\|_{\infty}\right)^{\beta_{1} \vee \beta_{2}} \\
& \times\left(\left\|Y_{0}-\widetilde{Y_{0}}\right\|_{V}+\left\|\dot{Y}_{0}-\dot{\widetilde{Y}}\right\|_{V}\right) \\
&+ 2 T^{\alpha}\left(1+T^{\alpha}\right)\left(\|\widetilde{\mathbf{W}}\|_{\mathscr{C}_{3}}+\|\widetilde{\mathbf{W}}\|_{\mathscr{C}_{3}}^{2}\right)\left(1+\|\dot{Y}\|_{\infty}+\|\dot{\tilde{Y}}\|_{\infty}\right)^{\beta_{0} \vee \beta_{1}+\beta_{1}} \\
&\left.\times\left(1+\|Y\|_{\infty}+\|\widetilde{Y}\|_{\infty}\right)^{\beta_{1} \vee \beta_{2}} d_{\alpha, W, \widetilde{W}}((Y, \dot{Y}),(\widetilde{Y}, \dot{\tilde{Y}}))\right\}|t-s|^{2 \alpha} .
\end{aligned}
$$

Recall inequality (3.32). Inequality (3.31) follows from (3.15), (3.40), (3.41) and the Sewing Lemma. This completes the proof of the proposition. 


\section{Rough Differential Equations}

Let $\alpha \in\left(\frac{1}{3}, \frac{1}{2}\right], \beta_{3}=\left(\beta_{0}, \ldots, \beta_{3}\right)$ where $\beta_{k} \geq 0, k=0, \ldots, 3$, and let $\mathbf{W}=(W, \mathbb{W}) \epsilon$ $\mathscr{C}^{\alpha, \beta_{3}}([0, T] \times V ; V)$. That is, $W$ is $\alpha$-Hölder in time, and three times differentiable in space with growth multi-index $\beta_{3}, \mathbb{W}$ is $2 \alpha$-Hölder in time and twice differentiable in space with growth multi-indexes $\beta_{2}^{*}=\left(\beta_{0}, \beta_{0} \vee \beta_{1}, \beta_{0} \vee \beta_{1} \vee \beta_{2}\right)$ and $\beta_{2}^{* *}=\left(\beta_{1}, \beta_{1} \vee\right.$ $\left.\beta_{2}, \beta_{1} \vee \beta_{2} \vee \beta_{3}\right)$, and $(W, \mathbb{W})$ satisfies Chen's relation (3.10). In this section, we study the following nonlinear RDE:

$$
Y_{t}=\xi+\int_{0}^{t} W\left(d r, Y_{r}\right)
$$

Definition 4.1. An $\alpha$-Hölder continuous function $Y$ is said to be a solution to (4.1), if $(Y, Y) \in \mathscr{E}_{W,(\xi, \xi)}^{2 \alpha}$, and equality (4.1) holds for all $t \in[0, T]$ where the integral on the right-hand side is a nonlinear rough integral in the sense of Theorem 3.10.

4.1. Local existence. In this section, we establish the (local) existence of a solution for equation (4.1) using Picard iteration method. To this end, we introduce the following notation. Let $\Phi \in \mathcal{C}^{\alpha}([0, T] ; V)$. For any $0 \leq s<t \leq T$, we write

$$
\|\Phi\|_{\alpha,[s, t]}:=\sup _{u \neq v \in[s, t]} \frac{\left\|\Phi_{u, v}\right\|_{V}}{|v-u|^{\alpha}} .
$$

We also define $d_{W, \alpha,[s, t]}$ in a similar way, where we recall Remark 3.8 for the definition of $d_{W, \alpha}$.

Theorem 4.2. For any $\xi \in V$, there exist a positive number $h$, such that the $R D E$ (4.1) has a solution $Y$ on $[0, h]$ with initial condition $Y_{0}=\xi$. In addition, the following inequality holds on $[0, h]$ :

$$
\|Y\|_{\alpha,[0, h]} \leq 5^{2 \gamma_{1}+2} k_{\alpha}\left(1+\|\mathbf{W}\|_{\mathscr{C}_{3}}\right)\left(1+\|\xi\|_{V}\right)^{\gamma_{1}},
$$

where $\gamma_{1}=\beta_{0} \vee \beta_{1}+\beta_{1} \vee \beta_{2}$.

Proof: Choose $h \in(0,1]$. Let

$$
\left(Y_{t}^{0}, \dot{Y}_{t}^{0}\right):=\left(\xi+W_{0, t}(\xi), \xi\right), \quad t \in[0, h] .
$$

Then $\left(Y^{0}, \dot{Y}^{0}\right) \in \mathscr{E}_{W,(\xi, \xi)}^{2 \alpha}$ with the remainder $R_{s, t}^{Y^{0}} \equiv 0$ for all $(s, t) \in[0, h]^{2}$. Due to Proposition 3.12, for any $n \geq 1$, we can recursively define an element $\left(Y^{n}, \dot{Y}^{n}\right) \epsilon$ $\mathscr{E}_{W,(\xi, \xi)}^{2 \alpha}$ given by

$$
Y_{t}^{n+1}=\xi+\int_{0}^{t} W\left(d r, Y_{t}^{n}\right), \quad t \in[0, h] .
$$

By (3.30), the following inequality holds for all $n \geq 1$

$$
\begin{gathered}
\left\|R^{Y^{n+1}}\right\|_{2 \alpha,[0, h]} \leq k_{\alpha}\|\mathbf{W}\|_{\mathscr{C}_{3}}\left(1+2\|\xi\|_{V}\right)^{\gamma_{1}}\left[1+2 h^{\alpha}\left(\left\|Y^{n}\right\|_{\alpha,[0, h]}+\left\|Y^{n-1}\right\|_{\alpha,[0, h]}\right)\right]^{\gamma_{1}} \\
\times\left[1+h^{\alpha}\left(\left\|Y^{n}\right\|_{\alpha,[0, h]}+\left\|Y^{n}\right\|_{\alpha,[0, h]}^{2}+\left\|Y^{n-1}\right\|_{\alpha,[0, h]}+\left\|R^{Y^{n}}\right\|_{2 \alpha,[0, h]}\right)\right] .
\end{gathered}
$$

By iteration, we know that $\left(Y^{n+1}, Y^{n}\right) \in \mathscr{E}_{W}^{2 \alpha,(\xi, \xi)}$, which implies that

$$
\left\|Y^{n+1}\right\|_{\alpha,[0, h]} \leq\|W\|_{\alpha, \beta_{3}}\left(1+\|\xi\|_{V}+h^{\alpha}\left\|Y^{n}\right\|_{\alpha,[0, h]}\right)^{\beta_{0}}+h^{\alpha}\left\|R^{Y^{n+1}}\right\|_{2 \alpha,[0, h]} .
$$


Choose $h_{1}=\left[5^{\gamma_{1}+2} k_{\alpha}\left(1+\|\mathbf{W}\|_{\mathscr{C}_{3}}\right)\left(1+2\|\xi\|_{V}\right)^{\gamma_{1}}\right]^{-\frac{1}{\alpha}}$. We claim that for any $h \epsilon$ $\left[0, h_{1}\right] \subset[0,1],\left\|Y^{n}\right\|_{\alpha,[0, h]}$ and $\left\|R^{n}\right\|_{2 \alpha,[0, h]}$ are bounded uniformly in $n$. To prove this claim, for any $h \in\left[0, h_{1}\right]$, let $f_{h}, g_{h}: \mathbb{R}_{+} \times \mathbb{R}_{+} \rightarrow \mathbb{R}_{+}$be given by

$$
f_{h}(x, y)=k_{\alpha}\|\mathbf{W}\|_{\mathscr{C}_{3}}\left(1+2\|\xi\|_{V}\right)^{\gamma_{1}}\left(1+4 h^{\alpha} y\right)^{\gamma_{1}}\left[1+h^{\alpha}\left(2 y+y^{2}+x\right)\right]
$$

and

$$
g_{h}(x, y)=\|W\|_{\alpha, \beta_{3}}\left(1+\|\xi\|_{V}+h^{\alpha} y\right)^{\beta_{0}}+h^{\alpha} x .
$$

Then it is easy to see that $f$ and $g$ are both increasing in each argument $h, x$ and $y$. Let

$$
x_{1}=\frac{\|\mathbf{W}\|_{\mathscr{C}_{3}}}{2\left(1+\|\mathbf{W}\|_{\mathscr{C}_{3}}\right)} h_{1}^{-2 \alpha} \quad \text { and } \quad y_{1}=\frac{\|\mathbf{W}\|_{\mathscr{C}_{3}}}{1+\|\mathbf{W}\|_{\mathscr{C}_{3}}} h_{1}^{-\alpha} .
$$

It follows that for any $h \in\left[0, h_{1}\right] \subset[0,1], x \in\left[0, x_{1}\right]$ and $y \in\left[0, y_{1}\right]$, the following inequalities hold

$$
\begin{aligned}
& f_{h}(x, y) \leq f_{h_{1}}\left(x_{1}, y_{1}\right) \\
& \quad=k_{\alpha}\|\mathbf{W}\|_{\mathscr{C}_{3}}\left(1+2\|\xi\|_{V}\right)^{\gamma_{1}} 5^{\gamma_{1}}\left(1+\frac{2\|\mathbf{W}\|_{\mathscr{C}_{3}}}{1+\|\mathbf{W}\|_{\mathscr{C}_{3}}}+\frac{\|\mathbf{W}\|_{\mathscr{C}_{3}}^{2} h^{-\alpha}}{\left(1+\|\mathbf{W}\|_{\mathscr{C}_{3}}\right)^{2}}+\frac{\|\mathbf{W}\|_{\mathscr{C}_{3}} h_{1}^{-\alpha}}{2\left(1+\|\mathbf{W}\|_{\mathscr{C}_{3}}\right)}\right) \\
& \quad \leq k_{\alpha}\|\mathbf{W}\|_{\mathscr{C}_{3}}\left(1+2\|\xi\|_{V}\right)^{\gamma_{1}} 5^{\gamma_{1}+1} h_{1}^{-\alpha}=\frac{\|\mathbf{W}\|_{\mathscr{C}_{3}}}{5\left(1+\|\mathbf{W}\|_{\mathscr{C}_{3}}\right)} h_{1}^{-2 \alpha} \leq x_{1}
\end{aligned}
$$

and

$$
g_{h}(x, y) \leq g_{h_{1}}\left(x_{1}, y_{1}\right) \leq 2\|W\|_{\alpha, \boldsymbol{\beta}_{3}}\left(1+\|\xi\|_{V}\right)^{\beta_{0}}+\frac{\|\mathbf{W}\|_{\mathscr{C}_{3}} h_{1}^{-\alpha}}{2\left(1+\|\mathbf{W}\|_{\mathscr{C}_{3}}\right)} \leq y_{1} .
$$

From inequalities (4.3) and (4.4) we can show, by a recursive argument, that

$$
\begin{aligned}
\max _{n \geq 0}\left\{\left\|Y^{n}\right\|_{\alpha,[0, h]}\right\} \leq g_{h_{1}}\left(x_{1}, y_{1}\right) & =5^{\gamma_{1}+2} k_{\alpha}\|\mathbf{W}\|_{\mathscr{C}_{3}}\left(1+2\|\xi\|_{V}\right)^{\gamma_{1}} \\
& \leq 5^{2 \gamma_{1}+2} k_{\alpha}\|\mathbf{W}\|_{\mathscr{C}_{3}}\left(1+\|\xi\|_{V}\right)^{\gamma_{1}}
\end{aligned}
$$

and

$$
\begin{aligned}
\max _{n \geq 0}\left\{\left\|R^{Y^{n}}\right\|_{2 \alpha,[0, h]}\right\} \leq x_{1} & =\frac{5^{2 \gamma_{1}+4}}{2} k_{\alpha}^{2}\|\mathbf{W}\|_{\mathscr{C}_{3}}\left(1+\|\mathbf{W}\|_{\mathscr{C}_{3}}\right)\left(1+2\|\xi\|_{V}\right)^{2 \gamma_{1}} \\
& \leq 5^{3 \gamma_{1}+4} k_{\alpha}^{2}\|\mathbf{W}\|_{\mathscr{C}_{3}}\left(1+\|\mathbf{W}\|_{\mathscr{C}_{3}}\right)\left(1+\|\xi\|_{V}\right)^{2 \gamma_{1}}
\end{aligned}
$$

provided that $\left\|Y^{0}\right\|_{\alpha,[0, h]},\left\|\dot{Y}^{0}\right\|_{\alpha,[0, h]} \leq y_{1}$ and $\left\|R^{Y^{0}}\right\|_{2 \alpha,[0, h]} \leq x_{1}$. Indeed, by definition, we know that $\left\|\dot{Y}^{0}\right\|_{\alpha,[0, h]}=\left\|R^{Y^{0}}\right\|_{2 \alpha,[0, h]}=0$, and

$$
\left\|Y^{0}\right\|_{\alpha,[0, h]} \leq\|W\|_{\alpha, \beta_{3}}\left(1+\|\xi\|_{V}\right)^{\gamma_{1}} \leq y_{1}
$$

As a consequence, we conclude that $\left\|Y^{n}\right\|_{\alpha,[0, h]}$ and $\left\|R^{n}\right\|_{2 \alpha,[0, h]}$ are bounded uniformly in $n$ for $h \in\left(0, h_{1}\right]$. This also yields that

$$
\max _{n \geq 0}\left\{\left\|Y^{n}\right\|_{\infty,[0, h]}\right\} \leq \frac{\|\mathbf{W}\|_{\mathscr{C}_{3}}}{1+\|\mathbf{W}\|_{\mathscr{C}_{3}}}+\|\xi\|_{V} \leq 1+\|\xi\|_{V}
$$

By (4.5), (4.7), Proposition 3.13 and the fact that $0<h \leq h_{1}=\left[5^{\gamma_{1}+2} k_{\alpha}(1+\right.$ $\left.\left.\| \mathbf{W}{\| \mathscr{C}_{3}}\right)\left(1+2\|\xi\|_{V}\right)^{\gamma_{1}}\right]^{-\frac{1}{\alpha}}<1$, we get the following estimate

$$
d_{\alpha, W,[0, h]}\left(\left(Y^{n+1}, Y^{n}\right),\left(Y^{n}, Y^{n-1}\right)\right) \leq C_{5} d_{\alpha, W,[0, h]}\left(\left(Y^{n}, Y^{n-1}\right),\left(Y^{n-1}, Y^{n-2}\right)\right),
$$


where

$$
\begin{aligned}
C_{5}= & 6 k_{\alpha} h^{\alpha}\left(1+h^{\alpha}\right)\left(1+\|\mathbf{W}\|_{\mathscr{C}_{3}}\right)^{2}\left(1+2\left\|Y^{n}\right\|_{\infty,[0, h]}+2\left\|Y^{n-1}\right\|_{\infty,[0, h]}\right)^{\beta_{2}^{* *}} \\
\times & \left(1+2\left\|Y^{n-1}\right\|_{\infty,[0, h]}+2\left\|Y^{n-2}\right\|_{\infty,[0, h]}\right)_{2}^{\beta_{2}^{*}+\beta_{1}} \\
\times & {\left[1+h^{\alpha}\left(2\left\|Y^{n-1}\right\|_{\alpha,[0, h]}+\left\|Y^{n}\right\|_{\alpha,[0, h]}+\left\|Y^{n-2}\right\|_{\alpha,[0, h]}\right)\right.} \\
& \left.+h^{2 \alpha}\left(\left\|Y^{n-1}\right\|_{\alpha,[0, h]}+\left\|Y^{n}\right\|_{\alpha,[0, h]}\right)^{2}+h^{2 \alpha}\left\|R^{Y^{n}}\right\|_{2 \alpha,[0, h]}\right] \\
\leq & 120 \times 5^{\beta_{2}^{* *}+\beta_{2}^{*}+\beta_{1}} k_{\alpha}\left(1+\|\mathbf{W}\|_{\mathscr{C}_{3}}\right)^{2}\left(1+\|\xi\|_{V}\right)^{\beta_{2}^{* *}+\beta_{2}^{*}+\beta_{1}} h^{\alpha} .
\end{aligned}
$$

Let $\gamma_{2}=\beta_{2}^{* *}+\beta_{2}^{*}+\beta_{1}=\max \left\{\beta_{0}, \beta_{1}, \beta_{2}\right\}+\max \left\{\beta_{1}, \beta_{2}, \beta_{3}\right\}+\beta_{1}$, and let $C_{6}=120 \times$ $5^{\gamma_{2}} k_{\alpha}$. Then, we have

$$
\begin{aligned}
& d_{\alpha, W,[0, h]}\left(\left(Y^{n+1}, Y^{n}\right),\left(Y^{n}, Y^{n-1}\right)\right) \\
\leq & C_{6}\left(1+\|\mathbf{W}\|_{\mathscr{C}_{3}}\right)^{2}\left(1+\|\xi\|_{V}\right)^{\gamma_{2}} h^{\alpha} d_{\alpha, W,[0, h]}\left(\left(Y^{n}, Y^{n-1}\right),\left(Y^{n-1}, Y^{n-2}\right)\right) .
\end{aligned}
$$

Choose $h_{2}=\left[2 C_{6}\left(1+\|\mathbf{W}\|_{\mathscr{C}_{3}}\right)^{2}\left(1+\|\xi\|_{V}\right)^{\gamma_{2}}\right]^{-\frac{1}{\alpha}} \leq h_{1} \leq 1$, and let $h \in\left(0, h_{2}\right]$. Then by (4.8), we have the following inequality

$$
d_{\alpha, W}\left(\left(Y^{n+1}, Y^{n}\right),\left(Y^{n}, Y^{n-1}\right)\right) \leq \frac{1}{2} d_{\alpha, W}\left(\left(Y^{n}, Y^{n-1}\right),\left(Y^{n-1}, Y^{n-2}\right)\right) .
$$

This yields that

$$
\sum_{n=1}^{\infty} d_{\alpha, W,[0, h]}\left(\left(Y^{n+1}, Y^{n}\right),\left(Y^{n}, Y^{n-1}\right)\right)<\infty .
$$

Due to Lemma 3.9, we can conclude that $\left(Y^{n}, Y^{n-1}\right) \rightarrow(Y, Y) \in \mathscr{E}_{W,(\xi, \xi)}^{2 \alpha}$ as $n \rightarrow \infty$. By Lemma 3.7 and Proposition 3.13, we have for any $0 \leq s \leq t \leq h$,

$$
\begin{aligned}
\left\|Y_{s, t}^{n+1}-\int_{s}^{t} W\left(d r, Y_{r}\right)\right\|_{V} & =\left\|\int_{s}^{t} W\left(d r, Y_{r}^{n}\right)-\int_{s}^{t} W\left(d r, Y_{r}\right)\right\|_{V} \\
& \leq C d_{\alpha, W,[0, h]}\left(\left(Y^{n}, Y^{n-1}\right),(Y, Y)\right)|t-s|^{\alpha},
\end{aligned}
$$

for some constant $C>0$ uniformly in $n$. This implies that equation (4.1) holds for all $t \in[0, h]$. Finally, inequality (4.2) follows from (4.5) and the fact that $(Y, Y)$ is the limit of $\left(Y^{n}, Y^{n-1}\right)$ in $\mathscr{E}_{W,(\xi, \xi)}^{2 \alpha}$.

4.2. Uniqueness and global existence. In this section, we prove the uniqueness of a solution for equation (4.1). We also present some hypotheses that imply the global existence of a solution for this equation.

Theorem 4.3. For any time interval $[0, T]$ and initial value $\xi \in V$. There exists at most one solution to equation (4.1).

Proof: Suppose that $Y$ and $\widetilde{Y}$ are two solutions to (4.1) with initial condition $\xi$ on $[0, T]$. By Proposition 3.13, the following inequality holds on $[0, h] \subset[0, T]$, assuming $h \leq 1$.

$$
d_{\alpha, W,[0, h]}((Y, Y),(\widetilde{Y}, \widetilde{Y})) \leq C_{5} d_{\alpha, W,[0, h]}((Y, Y),(\widetilde{Y}, \widetilde{Y}))
$$

where

$$
\begin{gathered}
C_{5}=12 k_{\alpha} h^{\alpha}\left(1+\|\mathbf{W}\|_{\mathscr{C}_{3}}\right)^{2}\left(1+2\|Y\|_{\infty}+2\|\widetilde{Y}\|_{\infty}\right)^{\beta_{2}^{* *}}\left(1+2\|\dot{Y}\|_{\infty}+2\|\dot{\widetilde{Y}}\|_{\infty}\right)^{\beta_{2}^{*}+\beta_{1}} \\
\times\left[1+\left(\|Y\|_{\alpha}+\|\dot{Y}\|_{\alpha}+\|\widetilde{Y}\|_{\alpha}+\|\dot{\widetilde{Y}}\|_{\alpha}\right)+\left(\|Y\|_{\alpha}+\|\widetilde{Y}\|_{\alpha}\right)^{2}+\left\|R^{Y}\right\|_{2 \alpha}\right]
\end{gathered}
$$


Choosing $h$ small enough, (4.9) yields that $Y \equiv \widetilde{Y}$ on $[0, h]$. Notice that the choice of $h$ doesn't dependent on the initial value. Therefore, by iteration, we can extend the uniqueness to any time interval $[0, T]$.

As stated in Section 2, the linear growth of the vector field cannot guarantee the global existence of a RDE driven by a linear rough path. This is also true in the case of nonlinear rough paths. In order to obtain the global existence, we introduce the following growth condition of $W$. Let $\mathbf{W}=(W, \mathbb{W}) \in \mathscr{C}^{\alpha, \beta_{3}}([0, T] \times V ; V)$, let $\gamma_{1}=\beta_{0} \vee \beta_{1}+\beta_{1} \vee \beta_{2}$, and let $\gamma_{2}=\max \left\{\beta_{0}, \beta_{1}, \beta_{2}\right\}+\max \left\{\beta_{1}, \beta_{2}, \beta_{3}\right\}+\beta_{1}$.

Hypothesis 1. $\frac{\gamma_{2}}{\alpha}-\gamma_{2}+\gamma_{1} \leq 1$.

A similar condition in the linear situation can be seen, e.g., in Besalú and Nualart (2011); Davie (2008); Lejay (2009).

Theorem 4.4. Under Hypothesis 1, the RDE (4.1) has a solution on any time interval $[0, T]$. By Theorem 4.3, this solution is unique.

Proof: Let $\epsilon_{1}=\left[2 C_{6}\left(1+\|\mathbf{W}\|_{\mathscr{C}_{3}}\right)^{2}\left(1+\|\xi\|_{V}\right)^{\gamma_{2}}\right]^{-\frac{1}{\alpha}}$ where $C_{6}=120 \times 5^{\gamma_{2}} k_{\alpha}$ is the constant appearing in (4.8). Then, by Theorem 4.2, the RDE has a solution $Y^{(1)}$ on $\left[0, \epsilon_{1}\right]$ with initial condition $Y_{0}^{(1)}=\xi$. We denote by $\xi_{1}=Y_{\epsilon_{1}}^{(1)}$ the terminal value of $Y$. In order to extend the solution to the entire interval $[0, T]$, we consider the following $\mathrm{RDE}$

$$
Y_{t}=Y_{s}+\int_{s}^{t} W\left(d r, Y_{r}\right)+\int_{s}^{t}\left(d r, Y_{r}\right)
$$

By Theorem 4.2 again, equation (4.10) has a solution $Y^{(2)}$ on $\left[\epsilon_{1}, \epsilon_{1}+\epsilon_{2}\right]$ with initial condition $Y_{\epsilon_{1}}=\xi_{1}$, where $\epsilon_{2}=\left[2 C_{6}\left(1+\|\mathbf{W}\|_{\mathscr{C}_{3}}\right)^{2}\left(1+\left\|\xi_{1}\right\|_{V}\right)^{\gamma_{2}}\right]^{-\frac{1}{\alpha}}$. By iteration, we have a sequence $\left\{\epsilon_{n}\right\}_{n \geq 1}$ with values in $(0,1)$, such that the equation (4.10) has a solution $Y^{(n+1)}$ on $\left[\eta_{n}, \eta_{n+1}\right]:=\left[\sum_{k=1}^{n} \epsilon_{k}, \sum_{k=1}^{n+1} \epsilon_{n+1}\right]$ with initial condition $Y_{\eta_{n}}^{(n+1)}=\xi_{n}:=Y_{\eta_{n}}^{(n)}$ and $\epsilon_{n+1}=\left[2 C_{6}\left(1+\|\mathbf{W}\|_{\mathscr{C}_{3}}\right)^{2}\left(1+\left\|\xi_{n}\right\|_{V}\right)^{\gamma_{2}}\right]^{-\frac{1}{\alpha}}$. By (4.2) we have the following inequality

$$
\begin{aligned}
\left\|\xi_{n+1}\right\|_{V} \leq\left\|Y^{(n+1)}\right\|_{\infty} & \leq\left\|\xi_{n}\right\|_{V}+\epsilon_{n+1}^{\alpha}\left\|Y^{(n+1)}\right\|_{\alpha} \\
& \leq\left\|\xi_{n}\right\|_{V}+\frac{5^{2 \gamma_{1}+2}\|\mathbf{W}\|_{\mathscr{C}_{3}}}{2 C_{6}\left(1+\|\mathbf{W}\|_{\mathscr{C}_{3}}\right)^{2}}\left(1+\left\|\xi_{n}\right\|_{V}\right)^{\gamma_{1}-\gamma_{2}} .
\end{aligned}
$$

Recall the assumption $\frac{\gamma_{2}}{\alpha}-\gamma_{2}+\gamma_{1} \leq 1$. By the mean value theorem for real valued functions, there exist $\tau \in[0,1]$, such that

$$
\begin{aligned}
\left(1+\left\|\xi_{n+1}\right\|_{V}\right)^{\frac{\gamma_{2}}{\alpha}} \leq & {\left[1+\left\|\xi_{n}\right\|_{V}+\frac{5^{2 \gamma_{1}+2}\|\mathbf{W}\|_{\mathscr{C}_{3}}}{2 C_{6}\left(1+\|\mathbf{W}\|_{\mathscr{C}_{3}}\right)^{2}}\left(1+\left\|\xi_{n}\right\|_{V}\right)^{\gamma_{1}-\gamma_{2}}\right]^{\frac{\gamma_{2}}{\alpha}} } \\
= & \left(1+\left\|\xi_{n}\right\|_{V}\right)^{\frac{\gamma_{2}}{\alpha}}+\left[5^{2 \gamma_{1}+2}\left(2 C_{6}\right)^{-1}\left(1+\left\|\xi_{n}\right\|_{V}\right)^{\gamma_{1}-\gamma_{2}}\right] \\
& \times \frac{\gamma_{2}}{\alpha}\left[1+\left\|\xi_{n}\right\|_{V}+\tau 5^{2 \gamma_{1}+2}\left(2 C_{6}\right)^{-1}\left(1+\left\|\xi_{n}\right\|_{V}\right)^{\gamma_{1}-\gamma_{2}}\right]^{\frac{\gamma_{2}}{\alpha}-1} .
\end{aligned}
$$

By definition we know that $\gamma_{1} \leq \gamma_{2}$. This implies

$$
\begin{aligned}
{\left[1+\left\|\xi_{n}\right\|_{V}+\tau 5^{2 \gamma_{1}+2}\left(2 C_{6}\right)^{-1}\left(1+\left\|\xi_{n}\right\|_{V}\right)^{\gamma_{1}-\gamma_{2}}\right]^{\frac{\gamma_{2}}{\alpha}-1} } \\
\leq\left(1+\left\|\xi_{n}\right\|_{V}\right)^{\frac{\gamma_{2}}{\alpha}-1} \times \max \left\{1,\left[1+5^{2 \gamma_{1}+2}\left(2 C_{6}\right)^{-1}\right]^{\frac{\gamma_{2}}{\alpha}-1}\right\} \\
=\left[1+5^{2 \gamma_{1}+2}\left(2 C_{6}\right)^{-1}\right]^{0 \vee\left(\frac{\gamma_{2}}{\alpha}-1\right)}\left(1+\left\|\xi_{n}\right\|_{V}\right)^{\frac{\gamma_{2}}{\alpha}-1} .
\end{aligned}
$$


As a consequence of inequalities (4.11) and (4.12), under the assumption 1, we can write

$$
\begin{aligned}
\left(1+\left\|\xi_{n+1}\right\|_{V}\right)^{\frac{\gamma_{2}}{\alpha}} & \leq\left(1+\left\|\xi_{n}\right\|_{V}\right)^{\frac{\gamma_{2}}{\alpha}}+\frac{\gamma_{2}}{\alpha}\left[1+5^{2 \gamma_{1}+2}\left(2 C_{6}\right)^{-1}\right]^{\frac{\gamma_{2}}{\alpha} \vee 1}\left(1+\left\|\xi_{n}\right\|_{V}\right)^{\gamma_{1}-\gamma_{2}+\frac{\gamma_{2}}{\alpha}-1} \\
& \leq\left(1+\left\|\xi_{n}\right\|_{V}\right)^{\frac{\gamma_{2}}{\alpha}}+\frac{\gamma_{2}}{\alpha}\left[1+5^{2 \gamma_{1}+2}\left(2 C_{6}\right)^{-1}\right]^{\frac{\gamma_{2}}{\alpha} \vee 1}
\end{aligned}
$$

It follows that

$$
\begin{aligned}
\epsilon_{n+1} & \geq\left[2 C_{6}\left(1+\|\mathbf{W}\|_{\mathscr{C}_{3}}\right)^{2}\right]^{-\frac{1}{\alpha}}\left[\left(1+\left\|\xi_{n-1}\right\|_{V}\right)^{\frac{\gamma_{2}}{\alpha}}+\frac{\gamma_{2}}{\alpha}\left[1+5^{2 \gamma_{1}+2}\left(2 C_{6}\right)^{-1}\right]^{\frac{\gamma_{2}}{\alpha} \vee 1}\right]^{-1} \\
& =\left[\epsilon_{n}^{-1}+\left(2 C_{6}\left(1+\|\mathbf{W}\|_{\mathscr{C}_{3}}\right)^{2}\right)^{\frac{1}{\alpha}} \frac{\gamma_{2}}{\alpha}\left[1+5^{2 \gamma_{1}+2}\left(2 C_{6}\right)^{-1}\right]^{\frac{\gamma_{2}}{\alpha} \vee 1}\right]^{-1} \\
& :=\left(\epsilon_{n}^{-1}+K_{0}\right)^{-1} .
\end{aligned}
$$

Observe that the constant $K_{0}$ is independent of $n$. Thus by iteration, the following inequality holds

$$
\sum_{n=1}^{\infty} \epsilon_{n} \geq \sum_{n=0}^{\infty} \frac{1}{\epsilon_{1}^{-1}+n K_{0}}=\infty .
$$

In other words, we can extend the solution to any time interval $[0, T]$.

Assume that the derivatives of $W$ are all bounded, that is $\beta_{3}=\left(\beta_{0}, 0,0,0\right)$. Then, Hypothesis 1 is equivalent to $\beta_{0} \leq \alpha$ and it coincides with Besalú and Nualart's condition for global existence (see Theorem 4.1 of Besalú and Nualart, 2011).

4.3. Properties of the solutions. Assume Hypothesis 1. In this section, we prove some properties of the solution to the RDE (4.1). The first proposition below provides an estimate for the Hölder norm of the solution to (4.1). Before stating the proposition, we first prove the following lemma.

Lemma 4.5. Suppose that $X \in \mathcal{C}^{\alpha}([0, T] ; V)$. Let $\pi=\left(0=t_{0}<t_{1}<t_{2}<\ldots, t_{n}=T\right)$ be a partition. Then,

$$
\|X\|_{\alpha} \leq n^{1-\alpha} \max _{1 \leq k \leq n}\|X\|_{\alpha,\left[t_{k}-t_{k-1}\right]} \leq(T /|\pi|)^{1-\alpha} \max _{1 \leq k \leq n}\|X\|_{\alpha,\left[t_{k}-t_{k-1}\right]} .
$$

Proof: For any $0 \leq s<t \leq T$. There exists $0 \leq k_{1} \leq k_{2} \leq n$ such that $s \leq k_{1} \leq k_{2} \leq t$. Then by Jensen's inequality for convex function $f(x)=|x|^{\frac{1}{\alpha}}$, we have

$$
\begin{aligned}
\frac{\left\|X_{s, t}\right\|_{V}}{|t-s|^{\alpha}} & \leq \frac{\left\|X_{s, t_{k_{1}}}\right\|_{V}+\left\|X_{t_{k_{1}}, t_{k_{1}+1}}\right\|_{V}+\cdots+\left\|X_{t_{k_{2}}, t}\right\|_{V}}{|t-s|^{\alpha}} \\
& \leq \max _{1 \leq k \leq n}\|X\|_{\alpha,\left[t_{k}-t_{k-1}\right]} \times \frac{\left|t_{k_{1}}-s\right|^{\alpha}+\cdots+\left|t-t_{k_{2}}\right|^{\alpha}}{|t-s|^{\alpha}} \\
& \leq \max _{1 \leq k \leq n}\|X\|_{\alpha,\left[t_{k}-t_{k-1}\right]} \times \frac{n^{1-\alpha}|t-s|^{\alpha}}{|t-s|^{\alpha}} .
\end{aligned}
$$

The lemma is then proved.

Proposition 4.6. Assume Hypothesis 1. Let $Y$ be the solution to the RDE (4.1) with initial condition $\xi \in V$. Then the following estimate holds:

$$
\|Y\|_{\alpha} \leq c\|\mathbf{W}\|_{\mathscr{C}_{3}}\left(1+\|\xi\|_{V}\right)^{\gamma_{1}+\frac{1-\alpha}{\alpha} \gamma_{2}} e^{\left(\frac{\alpha \gamma_{1}}{\gamma_{2}}+1-\alpha\right) K_{0} T}
$$


for some $c$ depending on $\alpha$ and $\beta_{3}$, where

$$
K_{0}=\left(2 C_{6}\left(1+\|\mathbf{W}\|_{\mathscr{C}_{3}}\right)^{2}\right)^{\frac{1}{\alpha}} \frac{\gamma_{2}}{\alpha}\left[1+5^{2 \gamma_{1}+2}\left(2 C_{6}\right)^{-1}\right]^{\frac{\gamma_{2}}{\alpha} \vee 1}
$$

and $C_{6}=120 \times 5^{\gamma_{2}} k_{\alpha}$ are the same as in (4.14) and (4.8), respectively.

Proof: Let $\epsilon_{1}=\left[2 C_{6}\left(1+\|\mathbf{W}\|_{\mathscr{C}_{3}}\right)^{2}\left(1+\|\xi\|_{V}\right)^{\gamma_{2}}\right]^{-\frac{1}{\alpha}}$. Theorems 4.2 and 4.3 imply that there exists a unique solution to (4.1) with initial condition $Y_{0}=\xi$ on $\left[0, \epsilon_{1}\right]$. Denote the solution by $Y^{(1)}$. Then, proceeding with a similar argument as in Theorem 4.4, we obtain a sequence $\left\{Y^{(n+1)}\right\}_{n \geq 1}$, where $Y^{(n+1)}$ is the unique solution to RDE (4.10) on $\left[\eta_{n}, \eta_{n+1}\right]=\left[\sum_{k=1}^{n} \epsilon_{k}, \sum_{k=1}^{n+1} \epsilon_{k}\right]$ with initial condition $Y_{\eta_{n}}^{(n+1)}:=\xi_{n}=Y_{\eta_{n}}^{(n)}$ and $\epsilon_{n+1}=\left[2 C_{6}\left(1+\|\mathbf{W}\|_{\mathscr{C}_{3}}\right)^{2}\left(1+\left\|\xi_{n}\right\|_{V}\right)^{\gamma_{2}}\right]^{-\frac{1}{\alpha}}$. By inequalities (4.2), (4.13) and an iteration argument, we have the following estimate:

$$
\left\|Y^{(n+1)}\right\|_{\alpha} \leq 5^{2 \gamma_{1}+2} k_{\alpha}\|\mathbf{W}\|_{\mathscr{C}_{3}}\left\{\left(1+\|\xi\|_{V}\right)^{\frac{\gamma_{2}}{\alpha}}+\frac{(n+1) \gamma_{2}}{\alpha}\left[1+5^{2 \gamma_{1}+2}\left(2 C_{6}\right)^{-1}\right]^{\frac{\gamma_{2}}{\alpha} \vee 1}\right\}^{\frac{\alpha \gamma_{1}}{\gamma_{2}}} .
$$

In order to obtain (4.16), we consider the following two cases. Firstly, if $T \leq \epsilon_{1}$, then (4.16) holds by taking $n=0$ in (4.17). On the other hand, for any $T>\epsilon_{1}$, there exists a positive integer $N$, such that $\eta_{N} \leq T \leq \eta_{N+1}$. Notice that by (4.15), we have

$$
T \geq \sum_{n=1}^{N} \epsilon_{n} \geq \sum_{n=1}^{N}\left(\epsilon_{1}^{-1}+K_{0} n\right)^{-1} \geq \frac{1}{K_{0}}\left(\log \left(\epsilon_{1}^{-1}+K_{0} N\right)-\log \left(\epsilon_{1}^{-1}\right)\right) .
$$

Recall that $\epsilon_{1}=\left[2 C_{6}\left(1+\|\mathbf{W}\|_{\mathscr{C}_{3}}\right)^{2}\left(1+\|\xi\|_{V}\right)^{\gamma_{2}}\right]^{-\frac{1}{\alpha}}$ and

$$
K_{0}=\left(2 C_{6}\left(1+\|\mathbf{W}\|_{\mathscr{C}_{3}}\right)^{2}\right)^{\frac{1}{\alpha}} \frac{\gamma_{2}}{\alpha}\left[1+5^{2 \gamma_{1}+2}\left(2 C_{6}\right)^{-1}\right]^{\frac{\gamma_{2}}{\alpha} \vee 1} .
$$

It follows that

$$
\begin{aligned}
N & \leq \frac{1}{K_{0}}\left(e^{K_{0} T+\log \left(\epsilon_{1}^{-1}\right)}-\epsilon_{1}^{-1}\right)=K_{0}^{-1}\left(e^{K_{0} T}-1\right)\left[2 C_{6}\left(1+\|\mathbf{W}\|_{\mathscr{C}_{3}}\right)^{2}\left(1+\|\xi\|_{V}\right)^{\gamma_{2}}\right]^{\frac{1}{\alpha}} \\
& =\frac{\alpha}{\gamma_{2}}\left[1+5^{2 \gamma_{1}+2}\left(2 C_{6}\right)^{-1}\right]^{-\left(\frac{\gamma_{2}}{\alpha} \vee 1\right)}\left(1+\|\xi\|_{V}\right)^{\frac{\gamma_{2}}{\alpha}}\left(e^{K_{0} T}-1\right) .
\end{aligned}
$$

Let $Y$ be the solution to (4.1) on $[0, T]$ with initial condition $\xi$. Then, combining Lemma 4.5, (4.17) and (4.18), we have

$$
\|Y\|_{\alpha} \leq N^{1-\alpha} \max _{1 \leq n \leq N+1}\left\|Y^{(n)}\right\|_{\alpha} \leq c\left(1+\|\xi\|_{V}\right)^{\gamma_{1}+\frac{1-\alpha}{\alpha} \gamma_{2}} e^{\left(\frac{\alpha \gamma_{1}}{\gamma_{2}}+1-\alpha\right) K_{0} T}
$$

for some $c$ depending on $\alpha$ and $\beta_{3}$. This completes the proof of the proposition.

The next proposition provides the dependency of the solution to (4.1) on the initial condition under Hypothesis 1.

Proposition 4.7. Assume that $\mathbf{W}=(W, \mathbb{W})$ satisfies the conditions in Theorem 4.4. Let $Y$ and $\widetilde{Y}$ be the solutions to the RDE (4.1) with initial conditions $\xi$ and $\widetilde{\xi}$, respectively. Then the following estimate holds

$$
d_{\alpha, W}((Y, Y),(\widetilde{Y}, \widetilde{Y})) \leq c^{T}\left(T^{1-\alpha} \vee 1\right)\|\xi-\widetilde{\xi}\|_{V}
$$

where $c$ is a constant depending on $\alpha, \beta_{3},\|\mathbf{W}\|_{\mathscr{C}_{3}}, \xi$ and $\widetilde{\xi}$. 
Proof: By Propositions 3.13 and 4.6, and the fact that $Y$ and $\widetilde{Y}$ are solutions to (4.1), we can write for any $h \in[0,1]$,

$$
\left.d_{\alpha, W,[0, h]}((Y, Y),(\widetilde{Y}, \widetilde{Y})) \leq c_{1}\|\xi-\widetilde{\xi}\|_{V}+c_{2} h^{\alpha} d_{\alpha, W,[0, h]}((Y, Y),(\widetilde{Y}, \widetilde{Y}))\right],
$$

where $c_{1}, c_{2}$ are constants depending on $\|\mathbf{W}\|_{\mathscr{C}_{3}}, \alpha, \beta_{3}$ and $\xi, \widetilde{\xi}$. Let $\epsilon=\left(2 c_{1}\right)^{-\frac{1}{\alpha}} \wedge$ $\left(2 c_{2}\right)^{-\frac{1}{\alpha}} \wedge 1$. It follows that

$$
d_{\alpha, W,[0, \epsilon]}((Y, Y),(\widetilde{Y}, \widetilde{Y})) \leq 2 c_{1}\|\xi-\widetilde{\xi}\|_{V}
$$

on $[0, \epsilon]$. By iteration, we have that for any $n \geq 1$,

$$
d_{\alpha, W,[n \epsilon,(n+1) \epsilon]}((Y, Y),(\widetilde{Y}, \widetilde{Y})) \leq 2 c_{1}\left\|Y_{n \epsilon}-\widetilde{Y}_{n \epsilon}\right\|_{V}
$$

and

$$
\begin{aligned}
\left\|Y_{n \epsilon}-\widetilde{Y}_{n \epsilon}\right\|_{V} & \leq\left\|Y_{(n-1) \epsilon}-\widetilde{Y}_{(n-1) \epsilon}\right\|_{V}+\epsilon^{\alpha}\|Y-\widetilde{Y}\|_{\alpha,[(n-1) \epsilon, n \epsilon]} \\
& \leq\left\|Y_{(n-1) \epsilon}-\widetilde{Y}_{(n-1) \epsilon}\right\|_{V}+\epsilon^{\alpha} d_{\alpha, W,[(n-1) \epsilon, n \epsilon]}((Y, Y),(\widetilde{Y}, \widetilde{Y})) \\
& \leq 2\left\|Y_{(n-1) \epsilon}-\widetilde{Y}_{(n-1) \epsilon}\right\|_{V} .
\end{aligned}
$$

Thus we can write

$$
d_{\alpha, W,[n \epsilon,(n+1) \epsilon]}((Y, Y),(\widetilde{Y}, \widetilde{Y})) \leq 2^{n+1} c_{1}\|\xi-\widetilde{\xi}\|_{V} .
$$

In order to obtain the global distance, we proceed as follows. If $T \leq \epsilon$, then (4.19) is a direct consequence of (4.21). It suffices to consider the case when $T>\epsilon$. Let $N$ be the positive integer such that $N \epsilon<T \leq(N+1) \epsilon$. Due to Lemma 4.5, the following inequality holds

$$
\begin{aligned}
d_{\alpha, W}((Y, Y),(\widetilde{Y}, \widetilde{Y})) & \leq(T / \epsilon)^{1-\alpha} \max _{0 \leq n \leq N}\left\{d_{\alpha, W,[n \epsilon,(n+1) \epsilon]}((Y, Y),(\widetilde{Y}, \widetilde{Y}))\right\} \\
& \leq c^{T} T^{1-\alpha}\|\xi-\widetilde{\xi}\|_{V},
\end{aligned}
$$

for some $c>0$ depending on $\alpha, \beta_{3},\|\mathbf{W}\|_{\mathscr{C}}, \xi$ and $\widetilde{\xi}$. This completes the proof of the proposition.

Due to Propositions 4.6 and 4.7, we can deduce the following corollary.

Corollary 4.8. Assume Hypothesis 1. Write $Y(\xi)$ for the solution to the RDE (4.1) with initial condition $\xi \in V$. Let $K$ be any positive constant. Then,

(i) $\|Y(\xi)\|_{\alpha}$ is uniformly bounded in the space $\left\{\xi,\|\xi\|_{V} \leq K\right\}$.

(ii) The constant $c$ in (4.19) is fixed in the space $\left\{(\xi, \widetilde{\xi}),\|\xi\|_{V}+\|\widetilde{\xi}\|_{V} \leq K\right\}$.

Remark 4.9. As a consequence of Proposition 4.7, we have the following estimates

$$
\|Y-\widetilde{Y}\|_{\alpha} \leq c^{T}\left(T^{1-\alpha} \vee 1\right)\|\xi-\widetilde{\xi}\|_{V}
$$

and

$$
\sup _{t \in[0, T]}\left\|Y_{t}-\widetilde{Y}_{t}\right\|_{V} \leq\left[1+c^{T}(T \vee 1)\right]\|\xi-\widetilde{\xi}\|_{V} .
$$

for some constants $c$ depending on $\alpha, \beta_{3},\|\mathbf{W}\|_{\mathscr{C}}, \xi$ and $\widetilde{\xi}$. 


\section{A functional approach to nonlinear rough paths}

Let $V$ be a Banach space. In this section, we consider the nonlinear rough path defined in Section 3 as a $\mathcal{C}^{\boldsymbol{\beta}_{n}}(V ; V)$-valued linear rough path. We will show that the two approaches are equivalent under some assumptions.

We start this section by defining the space $\mathcal{C}^{\beta_{n}}(V ; V)$ :

Definition 5.1. Let $\beta_{n}=\left(\beta_{0}, \ldots, \beta_{n}\right)$ be a multi-index, where $\beta_{k} \geq 0$ for all $k \epsilon$ $\{0,1, \ldots, n\}$. The space $\mathcal{C}^{\boldsymbol{\beta}_{n}}(V ; V)$ is the collection of continuously differentiable functions on $V$ with values in $V$, equipped with the norm:

$$
\|\phi\|_{\boldsymbol{\beta}_{n}}=\sum_{k=0}^{n} \sup _{x \in V} \frac{\left\|D^{k} \phi(x)\right\|_{\mathfrak{L}_{k}(V ; V)}}{\left(1+\|x\|_{V}\right)^{\beta_{k}}}<\infty .
$$

It is easy to see that $\left(\mathcal{C}^{\boldsymbol{\beta}_{n}}(V ; V),\|\cdot\|_{\boldsymbol{\beta}_{n}}\right)$ is a Banach space. In the next lemma, we show the equivalence of the spaces $\mathcal{C}^{\alpha}\left([0, T] ; \mathcal{C}^{\boldsymbol{\beta}_{n}}(V ; V)\right)$ and $\mathcal{C}^{\alpha, \boldsymbol{\beta}_{n}}([0, T] \times V ; V)$ defined in Definition 3.1.

Lemma 5.2. (i) Let $\Phi \in \mathcal{C}^{\alpha, \beta_{n}}([0, T] \times V ; V)$ be defined by (3.1) with $\Phi_{0} \in$ $\mathcal{C}^{\boldsymbol{\beta}_{n}}(V ; V)$. Then, $\Phi \in \mathcal{C}^{\alpha}\left([0, T] ; \mathcal{C}^{\boldsymbol{\beta}_{n}}(V ; V)\right)$.

(ii) Conversely, if $\Phi \in \mathcal{C}^{\alpha}\left([0, T] ; \mathcal{C}^{\boldsymbol{\beta}_{n}}(V ; V)\right)$, then $\Phi \in \mathcal{C}^{\alpha, \beta_{n}}([0, T] \times V ; V)$.

Proof: (i) Fix $t \in[0, T]$. We can show that

$$
\left\|\Phi_{t}\right\|_{\boldsymbol{\beta}_{n}} \leq\left\|\Phi_{0}\right\|_{\boldsymbol{\beta}_{n}}+\left\|\Phi_{0, t}\right\|_{\alpha, \boldsymbol{\beta}_{n}} \leq\left\|\Phi_{0}\right\|_{\boldsymbol{\beta}_{n}}+T^{\alpha}\|\Phi\|_{\alpha, \boldsymbol{\beta}_{n}}<\infty .
$$

Similarly for any $0 \leq s \leq t \leq T$, we have

$$
\left\|\Phi_{s, t}\right\|_{\boldsymbol{\beta}_{n}} \leq\|\Phi\|_{\alpha, \boldsymbol{\beta}_{n}}|t-s|^{\alpha} .
$$

It follows that as a $\mathcal{C}^{\boldsymbol{\beta}_{n}}(V ; V)$-valued function, $\|\Phi\|_{\alpha} \leq\|\Phi\|_{\alpha, \boldsymbol{\beta}_{n}}<\infty$.

(ii) We estimate $\|\Phi\|_{\alpha, \boldsymbol{\beta}_{n}}$ as follows:

$$
\|\Phi\|_{\alpha, \boldsymbol{\beta}_{n}}=\sum_{k=0}^{n} \sup _{\substack{s \neq t \in[0, T] \\ x \in V}} \frac{\left\|D^{k} \Phi_{s, t}(x)\right\|_{V}}{|t-s|^{\alpha}\left(1+\|x\|_{V}\right)^{\beta_{k}}}=\sup _{s \neq t \in[0, T]} \frac{\left\|\Phi_{s, t}\right\|_{\boldsymbol{\beta}_{n}}}{|t-s|^{\alpha}} \leq\|\Phi\|_{\alpha} .
$$

As a consequence, $\Phi \in \mathcal{C}^{\alpha, \beta_{n}}([0, T] \times V ; V)$.

Let $n \geq 1$, and let $(W, \mathcal{W}) \in \mathscr{C}^{\alpha}\left([0, T] ; \mathcal{C}^{\beta_{n}}(V ; V)\right)$ be a $\mathcal{C}^{\beta_{n}}(V ; V)$-valued linear rough path in the sense of Definition 2.2. Then, $\mathcal{W} \in \mathcal{C}_{2}^{2 \alpha}\left([0, T]^{2} ; \mathcal{C}^{\beta_{n}}(V ; V)^{\otimes 2}\right)$. We define $\mathbb{W}:[0, T]^{2} \times V^{2} \rightarrow V$ as follows:

$$
\mathbb{W}_{s, t}(x, y):=\mathcal{D}^{(2)} \mathcal{W}_{s, t}(x, y),
$$

where $\mathcal{D}^{(2)}: \mathcal{C}^{\boldsymbol{\beta}_{n}}(V ; V) \otimes \mathcal{C}^{\boldsymbol{\beta}_{n}}(V ; V) \rightarrow \mathcal{C}^{\boldsymbol{\beta}_{n-1}^{*}, \boldsymbol{\beta}_{n-1}^{* *}}(V \times V ; V)$ with the multi-indexes $\beta_{n-1}^{*}$ and $\beta_{n-1}^{* *}$ defined in (3.3), is given by

$$
\mathcal{D}^{(2)}\left(\phi^{1}, \phi^{2}\right)(x, y):=D \phi^{2}(y)\left(\phi^{1}(x)\right),
$$

for all $\left(\phi^{1}, \phi^{2}\right) \in \mathcal{C}^{\beta_{n}}(V ; V)^{2}$ and $(x, y) \in V^{2}$. One should notice that the operator $\mathcal{D}^{(2)}$ can be extended continuously to the tensor product space $\mathcal{C}^{\boldsymbol{\beta}_{n}}(V ; V) \otimes$ $\mathcal{C}^{\beta_{n}}(V ; V)$, and it is a linear operator on this space. We can also define

$$
\mathcal{D}^{(1)}\left(\phi^{1}, \phi^{2}\right)(x, y):=D \phi^{1}(x)\left(\phi^{2}(x)\right)
$$

for all $\left(\phi^{1}, \phi^{2}\right) \in \mathcal{C}^{\beta_{n}}(V ; V)^{2}$, and continuously extend it to $\mathcal{C}^{\beta_{n}}(V ; V) \otimes \mathcal{C}^{\beta_{n}}(V ; V)$. In the next proposition, we show that $(W, \mathbb{W}) \in \mathscr{C}^{\alpha, \boldsymbol{\beta}_{n}}([0, T] \times V ; V)$. 
Proposition 5.3. Let $\mathbf{W}=(W, \mathcal{W}) \in \mathscr{C}^{\alpha}\left([0, T] ; \mathcal{C}^{\boldsymbol{\beta}_{n}}(V ; V)\right)$, and let $\mathbb{W}:[0, T]^{2} \times$ $V^{2} \rightarrow V$ be given by (5.1). Then $(W, \mathbb{W}) \in \mathscr{C}^{\alpha, \boldsymbol{\beta}_{n}}([0, T] \times V ; V)$.

Proof: According to Lemma 5.2, we know that $W \in \mathcal{C}^{\alpha, \boldsymbol{\beta}_{n}}([0, T] \times V ; V)$ and thus $\mathbb{W} \in \mathcal{C}_{2}^{2 \alpha, \boldsymbol{\beta}_{n-1}^{*}, \boldsymbol{\beta}_{n-1}^{* *}}\left([0, T]^{2} \times V^{2} ; V\right)$. It suffices to verify Chen's relation (3.10). Recall that $(W, \mathcal{W}) \in \mathscr{C}^{\alpha}\left([0, T] ; \mathcal{C}^{\boldsymbol{\beta}_{n}}(V ; V)\right)$ satisfies Chen's relation $(2.3)$, and the operator $\mathcal{D}^{(2)}$ is linear on $\mathcal{C}^{\boldsymbol{\beta}_{n}}(V ; V) \otimes \mathcal{C}^{\boldsymbol{\beta}_{n}}(V ; V)$. It follows that

$$
\begin{array}{r}
\mathbb{W}_{s, t}(x, y)-\mathbb{W}_{s, u}(x, y)-\mathbb{W}_{u, t}(x, y)=\mathcal{D}^{(2)}\left(\mathcal{W}_{s, t}-\mathcal{W}_{s, u}-\mathcal{W}_{u, t}\right)(x, y) \\
=\mathcal{D}^{(2)}\left(W_{s, u} \otimes W_{u, t}\right)(x, y)=D W_{u, t}(y)\left(W_{s, u}(x)\right) .
\end{array}
$$

As a consequence, $(W, \mathbb{W}) \in \mathscr{C}^{\alpha, \boldsymbol{\beta}_{n}}([0, T] \times V ; V)$.

Remark 5.4. Proposition 5.3 shows that $\mathbb{W}$ can be constructed from $\mathcal{W}$. However, generally we are not able to recover $\mathcal{W}$ from $\mathbb{W}$ satisfying Chen's relation (3.10). In other words, the nonlinear integral $\int_{0}^{t} W\left(d r, Y_{r}\right)$ and the nonlinear RDE (4.1) can be studied using the approach of Section 3 even if $\mathcal{W}$ does not exist.

Let $\mathbf{W}=(W, \mathcal{W}) \in \mathscr{C}^{\alpha}\left([0, T] ; \mathcal{C}^{\beta_{n}}(V ; V)\right)$. In the theory of linear rough paths, under the assumption that $Y \in \mathscr{D}_{W}^{2 \alpha}\left(\mathcal{L}\left(\mathcal{C}^{\boldsymbol{\beta}_{n}}(V ; V) ; V\right)\right)$, the rough integral of $Y$ against $W$ is well-defined. The nonlinear rough integral defined in Section 3 can be also interpreted as this type of linear rough integral. In this case, the controlled rough path $Y$ belongs to a proper subset of $\mathscr{D}_{W}^{2 \alpha}\left(\mathcal{L}\left(\mathcal{C}^{\beta_{n}}(V ; V) ; V\right)\right)$, that is equivalent to $\mathscr{E}_{W}^{2 \alpha}$ in the sense of Definition 3.5. To describe this subset, we introduce the following special class of operators in $\mathcal{L}\left(\mathcal{C}^{\boldsymbol{\beta}_{n}}(V ; V) ; V\right)$. For any $x \in V$, let $\widehat{x}: \mathcal{C}^{\boldsymbol{\beta}_{n}}(V ; V) \rightarrow V$ be given by

$$
\widehat{x}(\phi):=\phi(x) .
$$

Then $\widehat{x} \in \mathcal{L}\left(\mathcal{C}^{\beta_{n}}(V ; V) ; V\right)$ with operator norm bounded by $\left(1+\|x\|_{V}\right)^{\beta_{0}}$. Let $n \geq 1$ and let $W \in \mathcal{C}^{\alpha}\left([0, T] ; \mathcal{C}^{\boldsymbol{\beta}_{n}}(V ; V)\right)$. We introduce the space of basic controlled rough paths of a $\mathcal{C}^{\boldsymbol{\beta}_{n}}(V ; V)$-valued rough path as a subspace of $\mathscr{D}_{W}^{2 \alpha}\left(\mathcal{L}\left(\mathcal{C}^{\boldsymbol{\beta}_{n}}(V ; V) ; V\right)\right)$, where the space $\mathscr{D}_{W}^{2 \alpha}\left(\mathcal{L}\left(\mathcal{C}^{\beta_{n}}(V ; V) ; V\right)\right)$ is defined as in Definition 2.3. Here, the state space of $W$ is $\mathcal{C}^{\beta_{n}}(V ; V)$. Let $\left(\mathcal{Y}, \mathcal{Y}^{\prime}\right) \in \mathscr{D}_{W}^{2 \alpha}\left(\mathcal{L}\left(\mathcal{C}^{\beta_{n}}(V ; V) ; V\right)\right)$. Then the state spaces of $\mathcal{Y}$ and $\mathcal{Y}^{\prime}$ are $\mathcal{L}\left(\mathcal{C}^{\boldsymbol{\beta}_{n}}(V ; V) ; V\right)$ and $\mathcal{L}\left(\mathcal{C}^{\boldsymbol{\beta}_{n}}(V ; V) ; \mathcal{L}\left(\mathcal{C}^{\boldsymbol{\beta}_{n}}(V ; V) ; V\right)\right)$, respectively.

Definition 5.5. A pair of functions $\left(\mathcal{Y}, \mathcal{Y}^{\prime}\right) \in \mathscr{D}_{W}^{2 \alpha}\left(\mathcal{L}\left(\mathcal{C}^{\boldsymbol{\beta}_{n}}(V ; V) ; V\right)\right)$ is called a basic rough path controlled by $W$, if there exists a pair of functions $(Y, \dot{Y}) \in \mathcal{C}^{\alpha}(V ; V) \times$ $\mathcal{C}^{\alpha}(V ; V)$, such that for all $t \in[0, T], \mathcal{Y}_{t}=\widehat{Y}_{t}$ and for all $\left(\phi_{1}, \phi_{2}\right) \in \mathcal{C}^{\boldsymbol{\beta}_{n}}(V ; V)^{2}$

$$
\mathcal{Y}_{t}^{\prime}\left(\phi_{1}, \phi_{2}\right)=\widehat{Y}_{t}^{\prime}\left(\phi_{1}, \phi_{2}\right):=\mathcal{D}^{(2)}\left(\phi_{1}, \phi_{2}\right)\left(\dot{Y}_{t}, Y_{t}\right)=D \phi_{2}\left(Y_{t}\right)\left(\phi_{1}\left(\dot{Y}_{t}\right)\right) \text {. }
$$

We write $\widetilde{\mathscr{E}}_{W}^{2 \alpha}$ for the collection of such pairs.

The next proposition provides the equivalence between the spaces $\widetilde{\mathscr{E}}_{W}^{2 \alpha}$ and $\mathscr{E}_{W}^{2 \alpha}$.

Proposition 5.6. Let $n \geq 1$ and let $W \in \mathcal{C}^{\alpha}\left([0, T] ; \mathcal{C}^{\boldsymbol{\beta}_{n}}(V ; V)\right)$. Then by Lemma 5.2, $W \in \mathcal{C}^{\alpha, \beta_{n}}([0, T] \times V ; V)$ as well. In addition, the following properties hold:

(i) Let $(Y, \dot{Y}) \in \mathscr{E}_{W}^{2 \alpha}$ in the sense of Definition 3.5. Then, $\left(\widehat{Y}, \widehat{Y}^{\prime}\right) \in \widetilde{\mathscr{E}}_{W}^{2 \alpha}$ in the sense of Definition 5.5, where $\widehat{Y}_{t}$ and $\widehat{Y}_{t}^{\prime}$ are given by (5.3) and (5.4) respectively, for all $t \in[0, T]$. 
(ii) Conversely, let $\left(\widehat{Y}, \widehat{Y}^{\prime}\right) \in \widetilde{\mathscr{E}}_{W}^{2 \alpha}$ with associated pair $(Y, \dot{Y}) \in \mathcal{C}^{\alpha}(V ; V)^{2}$. Then $(Y, \dot{Y}) \in \mathscr{E}_{W}^{2 \alpha}$

Proof: (i) By assumption $Y \in \mathcal{C}^{\alpha}([0, T] ; V)$. It follows that

$$
\begin{aligned}
\|\widehat{Y}\|_{\alpha} & =\sup _{s \neq t \in[0, T]} \frac{\left\|\widehat{Y}_{s, t}\right\|_{\mathcal{L}\left(\mathcal{C}^{\boldsymbol{\beta}_{n}}(V ; V) ; V\right)}}{|t-s|^{\alpha}}=\sup _{s \neq t \in[0, T]} \sup _{0 \neq \phi \in \mathcal{C}^{\boldsymbol{\beta}_{n}}(V ; V)} \frac{\left\|\phi\left(Y_{t}\right)-\phi\left(Y_{s}\right)\right\|_{V}}{|t-s|^{\alpha}\|\phi\|_{\boldsymbol{\beta}_{n}}} \\
& \leq\left(1+\|Y\|_{\infty}\right)^{\beta_{1}}\|Y\|_{\alpha} .
\end{aligned}
$$

This implies that $\widehat{Y} \in \mathcal{C}^{\alpha}\left([0, T] ; \mathcal{L}\left(\mathcal{C}^{\beta_{n}}(V ; V) ; V\right)\right)$. Similarly, since $\dot{Y} \in$ $\mathcal{C}^{\alpha}([0, T] ; V)$, we can deduce the following inequality:

$$
\left\|\widehat{Y}^{\prime}\right\|_{\alpha} \leq\left(1+\|Y\|_{\infty}\right)^{\beta_{2}}\left(1+\|\dot{Y}\|_{\infty}\right)^{\beta_{0}}\|Y\|_{\alpha}+\left(1+\|Y\|_{\infty}\right)^{\beta_{1}}\left(1+\|\dot{Y}\|_{\infty}\right)^{\beta_{1}}\|\dot{Y}\|_{\alpha} .
$$

It suffices to estimate the reminder term. Recall that $\widehat{Y}^{\prime}$ is defined as in (5.4). Then, for any $\phi \in \mathcal{C}^{\beta_{n}}(V ; V)$, the remainder $R_{s, t}^{\widehat{Y}}(\phi)$ can be written as follows,

$$
R_{s, t}^{\widehat{Y}}(\phi)=\phi\left(Y_{t}\right)-\phi\left(Y_{s}\right)-D \phi\left(Y_{s}\right) W_{s, t}\left(\dot{Y}_{s}\right) .
$$

Due to Taylor's Theorem 2.11 and the fact that $(Y, \dot{Y}) \in \mathscr{E}_{W}^{2 \alpha}$, we have

$$
\begin{aligned}
\left\|R_{s, t}^{\widehat{Y}}(\phi)\right\|_{V} \leq & \left\|D \phi\left(Y_{s}\right) Y_{s, t}-D \phi\left(Y_{s}\right) W_{s, t}\left(\dot{Y}_{s}\right)\right\|_{V} \\
& +\frac{1}{2} \sup _{0 \leq \tau \leq 1}\left\|D^{2} \phi\left(\tau Y_{t}+(1-\tau) Y_{s}\right)\left(Y_{s, t} Y_{s, t}\right)\right\|_{V} \\
= & \left\|D \phi\left(Y_{s}\right)\left[W_{s, t}\left(\dot{Y}_{s}\right)+R_{s, t}^{Y}\right]-D \phi\left(Y_{s}\right) W_{s, t}\left(\dot{Y}_{s}\right)\right\|_{V} \\
& +\frac{1}{2} \sup _{0 \leq \tau \leq 1}\left\|D^{2} \phi\left(\tau Y_{t}+(1-\tau) Y_{s}\right)\left(Y_{s, t} Y_{s, t}\right)\right\|_{V} \\
\leq & \|\phi\|_{\boldsymbol{\beta}_{n}}\left[\frac{1}{2}\left(1+\|Y\|_{\infty}\right)^{\beta_{2}}\|Y\|_{\alpha}^{2}+\left(1+\|Y\|_{\infty}\right)^{\beta_{1}}\left\|R^{Y}\right\|_{2 \alpha}\right]|t-s|^{2 \alpha} .
\end{aligned}
$$

This implies $R^{\widehat{Y}} \in \mathcal{C}_{2}^{2 \alpha}\left([0, T] ; \mathcal{L}\left(\mathcal{C}^{\boldsymbol{\beta}_{n}}(V ; V) ; V\right)\right)$. As a consequence, we conclude that $\left(\widehat{Y}, \widehat{Y}^{\prime}\right) \in \widetilde{\mathscr{E}}_{W}^{2 \alpha}$.

(ii) To prove the converse result, it suffices to show that $R^{Y} \in \mathcal{C}_{2}^{2 \alpha}([0, T] ; V)$, where

$$
R_{s, t}^{Y}:=Y_{s, t}-W_{s, t}\left(\dot{Y}_{s}\right)
$$

Let $K$ be the closed convex hull of the set $\left\{Y_{t}, t \in[0, T]\right\}$, and let $\widetilde{K}$ is a compact set in $V$ whose interior contains $K$. Choose a function $\phi: V \rightarrow V$ that is infinitely differentiable and satisfies the following properties:

a) $\phi(x)=x$ for all $x \in K$. That implies $D \phi(x)=I$ and $D^{2} \phi(x)=0$ for all $x \in K$, where $I$ denotes the identity operator in $\mathcal{L}(V ; V)$.

b) $\phi(x) \equiv x_{0} \in V$ for all $x \notin \widetilde{K}$.

c) $\phi$ and all its derivatives are bounded.

Then, it is easy to check that $\phi \in \mathcal{C}^{\boldsymbol{\beta}_{n}}(V ; V)$ for any multi-index $\boldsymbol{\beta}_{n}$. In addition, we can show that

$$
\begin{aligned}
\left\|R_{s, t}^{Y}\right\|_{V} & =\left\|\phi\left(Y_{t}\right)-\phi\left(Y_{s}\right)-D \phi\left(Y_{s}\right)\left[W_{s, t}\left(\phi_{s}\right)\right]\right\|_{V} \\
& =\left\|R_{s, t}^{\widehat{Y}}(\phi)\right\|_{V} \leq\left\|R^{\widehat{Y}}\right\|_{2 \alpha}\|\phi\|_{\beta_{n}}|t-s|^{2 \alpha} .
\end{aligned}
$$

In other words, $R^{Y} \in \mathcal{C}^{2 \alpha}([0, T] ; V)$, and thus $(Y, \dot{Y}) \in \mathscr{E}_{W}^{2 \alpha}$.

In the next theorem, we will show the equivalence of two rough integrals. 
Theorem 5.7. Let $\mathbf{W}=(W, \mathcal{W}) \in \mathscr{C}^{\alpha}\left([0, T] ; \mathcal{C}^{\beta_{2}}(V ; V)\right)$. Due to Proposition 5.3, we can construct $(W, \mathbb{W}) \in \mathscr{C}^{\alpha, \boldsymbol{\beta}_{2}}([0, T] \times V ; V)$. Assume that $\left(\widehat{Y}, \widehat{Y}^{\prime}\right) \in \widetilde{\mathscr{E}}_{W}^{2 \alpha}$ with associated pair $(Y, \dot{Y}) \in \mathscr{E}_{W}^{2 \alpha}$ by Proposition 5.6. Then, the following two rough integrals coincide,

$$
\int_{s}^{t} W\left(d r, Y_{r}\right)=\int_{s}^{t} \widehat{Y}_{r} d \mathbf{W}_{r}
$$

where the integral on the left-hand side is in the sense of (3.26), and the integral on the right-hand side is in the sense of Theorem 2.4.

Proof: Let $\Xi_{s, t}$ and $\widetilde{\Xi}_{s, t}$ be the approximations of the integral on the left and right-hand side, respectively. That is,

$$
\Xi_{s, t}=W_{s, t}\left(Y_{s}\right)+\mathbb{W}_{s, t}\left(\dot{Y}_{s}, Y_{s}\right) \text { and } \widetilde{\Xi}_{s, t}=\widehat{Y}_{s} W_{s, t}+\widehat{Y}_{s}^{\prime} \mathcal{W}_{s, t} .
$$

Here $\widehat{Y}_{s}^{\prime}$ acting on $\mathcal{W}_{s, t}$ is a continuous extension of formula (5.4) to the tensor product space $\mathcal{C}^{\beta_{2}}(V ; V)^{\otimes 2}$. By definition of $\mathbb{W}$ and $\left(\widehat{Y}, \widehat{Y}^{\prime}\right)$, we have

$$
\widehat{Y}_{s} W_{s, t}+\widehat{Y}_{s}^{\prime} \mathcal{W}_{s, t}=W_{s, t}\left(Y_{s}\right)-\mathcal{D}^{(2)} \mathcal{W}_{s, t}\left(\dot{Y}_{s}, Y_{s}\right)=W_{s, t}\left(Y_{s}\right)+\mathbb{W}_{s, t}\left(\dot{Y}_{s}, Y_{s}\right)
$$

This implies the equality (5.5).

At the end of this section, we provide an alternative approach to study the nonlinear RDE introduced in Section 4. Let $\mathbf{W}=(W, \mathcal{W}) \in \mathscr{C}^{\alpha}\left([0, T] ; \mathcal{C}^{\boldsymbol{\beta}_{3}}(V ; V)\right)$. Then, the RDE (4.1) can be also understood as the following equation:

$$
Y_{t}=\xi+\int_{0}^{t} \delta\left(Y_{r}\right) d \mathbf{W}_{r}
$$

where $\delta$ denotes the Dirac delta operator, that is $\delta: V \rightarrow \mathcal{L}\left(\mathcal{C}^{\beta_{3}}(V ; V) ; V\right)$ is given by $\delta(x)=\widehat{x}$. A function $Y \in \mathcal{C}^{\alpha}([0, T] ; V)$ is said to be a solution to (5.6), if $(Y, \delta(Y)) \in \mathscr{D}_{W}^{2 \alpha}(V)$ and the equality holds. On the other hand, suppose that $Y$ is a solution to (5.6). Then, $\left(\widehat{Y}, \widehat{Y}^{\prime}\right) \in \widetilde{\mathscr{E}}_{W}^{2 \alpha}$ with associated pair $(Y, Y) \in \mathscr{E}_{W}^{2 \alpha}$. Therefore, $Y$ is a solution to the equation (4.1) in the sense of Definition 4.1.

On the other hand, notice that as an $\mathcal{L}\left(\mathcal{C}^{\beta_{3}}(V ; V) ; V\right)$-valued operator, $\delta$ is three times differentiable. More precisely, the derivatives of $\delta$ can be written as follows $D^{k} \delta(x)(\phi)=D^{k} \phi(x)$ for $k=1,2,3$. Thus $\left\|D^{k} \delta(x)\right\| \leq\left(1+\|x\|_{V}\right)^{\beta_{k}}$ for all $k=0,1,2,3$. Then the (global) existence and uniqueness of equation (4.1) can be derived by the theory of linear rough paths (see e.g. Lejay, 2009). For other conditions that implies global existence, we refer the reader to the papers Lejay (2009, 2012). We did not consider Lejay's condition for global existence in Section 4, because we doubt whether it is applicable in our setting. Under the basic assumptions in Section 4, there may not exists $\mathcal{W}$ such that $\mathbb{W}=\mathcal{D}^{(2)} \mathcal{W}$. In this case, the result of linear rough path cannot be directly applied without any changes.

\section{Some applications of nonlinear rough paths}

6.1. An Itô-type formula for controlled rough paths. In this section, we follow the idea of Section 5 to consider the nonlinear rough path as a $\mathcal{C}^{\beta_{n}}(V ; V)$-valued rough path. Then, we aim to generalize the Itô-type formula (3.12) in Hu and Lê (2017) proved in the nonlinear Young's case. 
Theorem 6.1. Let $\mathbf{W}=(W, \mathcal{W}) \in \mathcal{C}^{\alpha}\left([0, T] ; \mathcal{C}^{\beta_{3}}(V ; V)\right)$. Assume that $\left(Y, Y^{\prime}\right) \in$ $\mathscr{D}_{W}^{2 \alpha}(V)$ and $\left(Z, Z^{\prime}\right) \in \mathscr{D}_{W}^{2 \alpha}(\mathcal{L}(V ; K))$. Then, the following Itô-type formula holds

$$
\begin{aligned}
\int_{s}^{t} Z_{r} d W\left(r, Y_{r}\right)=\int_{s}^{t} Z_{r} W\left(d r, Y_{r}\right)+\int_{s}^{t} Z_{r} D W\left(r, Y_{r}\right) d Y_{r} \\
+\frac{1}{2}\left[\int_{s}^{t} Z_{r} D^{2} W\left(r, Y_{r}\right) d\langle Y\rangle_{r}+\int_{s}^{t} Z_{r} d\left\langle\langle X, Y\rangle_{r}+\int_{s}^{t} Z_{r} d\left\langle\langle Y, X\rangle_{r}\right],\right.\right.
\end{aligned}
$$

where

$$
\begin{gathered}
X_{t}:=\int_{0}^{t} D W\left(d r, Y_{r}\right)=\lim _{|\pi| \rightarrow 0}\left[D W_{t_{k-1}, t_{k}}\left(Y_{t_{k-1}}\right)+\left(\mathcal{D}^{(2)}\right)^{2} Y_{t_{k-1}}^{\prime} \mathcal{W}_{t_{k-1}, t_{k}}\right], \\
\left(\mathcal{D}^{(2)}\right)^{2} Y_{t}^{\prime}\left(\phi_{1}, \phi_{2}\right):=D^{2} \phi_{2}\left(Y_{t}^{\prime} \phi_{1}\right) \in \mathcal{L}(V ; V) .
\end{gathered}
$$

The first three integrals in (6.1) are rough integrals in the sense of Proposition 2.7 (ii), while the last three integrals on the second line are Young's integral. In the above expressions, $\langle Y\rangle,\langle\langle X, Y\rangle$ and $\langle\langle Y, X\rangle$ are $2 \alpha$-continuous functions defined in Definition 2.9 and Remark 2.10.

Formula (6.1) provides the total differential $d W\left(t, Y_{t}\right)$ of $W\left(t, Y_{t}\right)$, that means, heuristically, $d W\left(t, Y_{t}\right)=\frac{d}{d t} W\left(t, Y_{t}\right) d t$. Comparing with the classical Itô lemma, the function $W$ in Theorem 6.1 is not differentiable, but only $\alpha$-Hölder continuous in time. In this case, the assumption that $Y$ is controlled by $W$ ensures that $W\left(d t, Y_{t}\right)$ is well-defined as the differential of the rough path $G_{t}=\int_{0}^{t} W\left(d r, Y_{r}\right)$ controlled by $W$.

In order to prove Theorem 6.1, we should make each integral in (6.1) to be well-defined. The first lemma below shows that $F_{t}=W\left(t, Y_{t}\right)$ is controlled by $W$.

Lemma 6.2. Let $W \in \mathcal{C}^{\alpha}\left([0, T] ; \mathcal{C}^{\beta_{2}}(V ; V)\right)$, and let $\left(Y, Y^{\prime}\right) \in \mathscr{D}_{W}^{2 \alpha}(V)$. Denote $F_{t}=W\left(t, Y_{t}\right)$. Then, $F \in \mathscr{D}_{W}^{2 \alpha}(V)$.

Proof: By Taylor's Theorem 2.11 and the fact that $\left(Y, Y^{\prime}\right) \in \mathscr{D}_{W}^{2 \alpha}(V)$, we get

$$
\begin{aligned}
F_{s, t} & =F_{t}-F_{s}=W_{s, t}\left(Y_{s}\right)+\left[W_{s, t}\left(Y_{t}\right)-W_{s, t}\left(Y_{s}\right)\right]+W_{s}\left(Y_{t}\right)-W_{s}\left(Y_{s}\right) \\
& =\widehat{Y}_{s} W_{s, t}+D W_{s}\left(Y_{s}\right)\left[Y_{s}^{\prime} W_{s, t}+R_{s, t}^{Y}\right]+O\left(\left\|Y_{s, t}\right\|_{V}^{2} \mid\right) .
\end{aligned}
$$

This yields that $\left(F, F^{\prime}\right) \in \mathscr{D}_{W}^{2 \alpha}(V)$, where $F^{\prime}:=\widehat{Y}+D W(Y) Y^{\prime} \in \mathcal{L}\left(\mathcal{C}^{\beta_{2}} ; V\right)$.

Suppose that $\mathbf{W}=(W, \mathcal{W}) \in \mathscr{C}^{\alpha}\left([0, T] ; \mathcal{C}^{\beta_{3}}(V ; V)\right)$. As a consequence of Lemma 6.2, the integral $\int_{s}^{t} Z_{r} d W\left(r, Y_{r}\right)=\int_{s}^{t} Z_{r} d F_{r}$ is well-defined as the integral of two controlled rough paths in the sense of (2.12). Additionally, by Taylor's Theorem 2.11, we can approximate this integral in the following way:

$$
\begin{array}{rl}
\int_{s}^{t} Z_{r} & d W\left(r, Y_{r}\right)=Z_{s} F_{s, t}+Z_{s}^{\prime} F_{s}^{\prime} \mathcal{W}_{s, t}+O\left(|t-s|^{3 \alpha}\right) \\
= & Z_{s} W_{s, t}\left(Y_{s}\right)+Z_{s} D W_{s, t}\left(Y_{s}\right) Y_{s, t}+Z_{s} D W_{s}\left(Y_{s}\right) Y_{s, t}+\frac{1}{2} Z_{s} D^{2} W_{s}\left(Y_{s}\right)\left(Y_{s, t}, Y_{s, t}\right) \\
& +Z_{s}^{\prime} \widehat{Y}_{s} \mathcal{W}_{s, t}+Z_{s}^{\prime} D W\left(s, Y_{s}\right) Y_{s}^{\prime} \mathcal{W}_{s, t}+O\left(|t-s|^{3 \alpha}\right)
\end{array}
$$

where

and

$$
Z_{s}^{\prime} \widehat{Y}_{s}\left(\phi_{1}, \phi_{2}\right)=Z_{s}^{\prime}\left(\phi_{1}\right)\left[\phi_{2}\left(Y_{s}\right)\right]
$$

$$
Z_{s}^{\prime} D W\left(s, Y_{s}\right) Y_{s}^{\prime}\left(\phi_{1}, \phi_{2}\right)=Z_{s}^{\prime}\left(\phi_{1}\right)\left[D W\left(s, Y_{s}\right)\left(Y_{s}^{\prime}\left(\phi_{2}\right)\right)\right],
$$

for all $\left(\phi_{1}, \phi_{2}\right) \in \mathcal{C}^{\beta_{3}}(V ; V)^{2}$. 
The next lemma provides a generalized version of Theorem 3.10. The proof is similar and we omit it.

Lemma 6.3. Let $(W, \mathcal{W}) \in \mathcal{C}^{\alpha}\left([0, T] ; \mathcal{C}^{\beta_{2}}(V ; V)\right)$, and let $\left(Y, Y^{\prime}\right) \in \mathscr{D}_{W}^{2 \alpha}(V)$. Then, the following limit exists and defines an additive function:

$$
\int_{s}^{t} W\left(d r, Y_{r}\right):=\lim _{|\pi| \rightarrow 0} \sum_{k=1}^{n}\left[W_{t_{k-1}, t_{k}}\left(Y_{s}\right)+Y_{t_{k-1}}^{\prime} \widehat{Y}_{t_{k-1}} \mathcal{D}^{(2)} \mathcal{W}_{t_{k-1}, t_{k}}\right],
$$

where $Y_{t}^{\prime} \widehat{Y}_{t} \mathcal{D}^{(2)}\left(\phi_{1}, \phi_{2}\right):=D \phi_{2}\left(Y_{t}\right)\left[Y_{t}^{\prime}\left(\phi_{1}\right)\right]$ for any $\left(\phi_{1}, \phi_{2}\right) \in \mathcal{C}^{\boldsymbol{\beta}_{2}}(V ; V)$.

For all $t \in[0, T]$, let $G_{t}:=\int_{0}^{t} W\left(d r, Y_{r}\right)$. Then, a similar argument as in Proposition 3.12 implies that $(G, Y) \in \mathscr{E}_{W}^{2 \alpha}$ or equivalently $(G, \widehat{Y}) \in \mathscr{D}_{W}^{2 \alpha}(V)$. Therefore, the integral $\int_{s}^{t} Z_{r} W\left(d r, Y_{r}\right)=\int_{s}^{t} Z_{r} d G_{r}$, defined as in (2.12), can be approximated in the following way,

$$
\begin{aligned}
\int_{s}^{t} Z_{r} W\left(d r, Y_{r}\right) & =Z_{s} G_{s, t}+Z_{s}^{\prime} \widehat{Y}_{s} \mathcal{W}_{s, t}+O\left(|t-s|^{3 \alpha}\right) \\
& =Z_{s} W_{s, t}\left(Y_{s}\right)+Z_{s} Y_{s}^{\prime} \widehat{Y}_{s} \mathcal{D}^{(2)} \mathcal{W}_{s, t}+Z_{s}^{\prime} \widehat{Y}_{s} \mathcal{W}_{s, t}+O\left(|t-s|^{3 \alpha}\right) .
\end{aligned}
$$

Assume that $(W, \mathcal{W}) \in \mathscr{C}^{\alpha}\left([0, T] ; \mathcal{C}^{\beta_{3}}(V ; V)\right)$. Let $H_{t}=Z_{t} D W\left(t, Y_{t}\right) \in \mathcal{L}(V ; V)$ for all $t \in[0, T]$. By a similar argument as in Lemma 6.2, we can show that

$$
H_{s, t}=Z_{s}^{\prime} W_{s, t} D W\left(s, Y_{s}\right)+Z_{s} \widehat{Y}_{s} D W_{s, t}+Z_{s} D^{2} W\left(s, Y_{s}\right) Y_{s}^{\prime} W_{s, t}+O\left(|t-s|^{2 \alpha}\right) .
$$

In other words, $H$ is controlled by $W$. This allows us to define $\int_{s}^{t} Z_{r} D W\left(r, Y_{r}\right) d Y_{r}=$ $\int_{s}^{t} H_{r} d Y_{r}$ by (2.12). In addition, we can approximate this integral as follows,

$$
\begin{aligned}
\int_{s}^{t} Z_{r} D W\left(r, Y_{r}\right) d Y_{r}= & Z_{s} D W\left(s, Y_{s}\right) Y_{s, t}+Z_{s}^{\prime} D W\left(s, Y_{s}\right) Y_{s}^{\prime} \mathcal{W}_{s, t} \\
& +Z_{s} \widehat{Y}_{s} \mathcal{D}^{(1)} Y_{s}^{\prime} \mathcal{W}_{s, t}+Z_{s} D^{2} W\left(s, Y_{s}\right) Y_{s}^{\prime} Y_{s}^{\prime} \mathcal{W}_{s, t}+O\left(|t-s|^{3 \alpha}\right)
\end{aligned}
$$

where $\mathcal{D}^{(1)}$ is defined as in (5.2),

$$
\begin{gathered}
Z_{s}^{\prime} D W\left(s, Y_{s}\right) Y_{s}^{\prime}\left(\phi_{1}, \phi_{2}\right)=Z_{s}^{\prime}\left(\phi_{1}\right)\left[D W\left(s, Y_{s}\right)\left(Y_{s}^{\prime}\left(\phi_{2}\right)\right)\right], \\
Z_{s} \widehat{Y}_{s} \mathcal{D}^{(1)} Y_{s}^{\prime}\left(\phi_{1}, \phi_{2}\right)=Z_{s}\left[D \phi_{1}\left(Y_{s}\right) Y_{s}^{\prime}\left(\phi_{2}\right)\right],
\end{gathered}
$$

and

$$
Z_{s} D^{2} W\left(s, Y_{s}\right) Y_{s}^{\prime} Y_{s}^{\prime}\left(\phi_{1}, \phi_{2}\right)=Z_{s}\left[D^{2} W\left(s, Y_{s}\right)\left(Y_{s}^{\prime}\left(\phi_{1}\right), Y_{s}^{\prime}\left(\phi_{2}\right)\right)\right],
$$

for all $\left(\phi_{1}, \phi_{2}\right) \in \mathcal{C}^{\boldsymbol{\beta}_{3}}(V ; V)$.

By a similar argument as in Theorem 3.10 and the Sewing Lemma, we can show that the limit in (6.2) uniquely exists. It allows us to define $X_{t}$ to be the limit. In addition, we can verify that $X \in \mathscr{D}_{W}^{2 \alpha}(\mathcal{L}(V ; V))$. Thus the three quadratic compensator terms on the second line of (6.1) are all well-defined, and according to Remark 2.10 (iii), $\langle Y\rangle \in \mathcal{C}_{2}^{2 \alpha}([0, T] ; V \otimes V)$ and $\left\langle\langle X, Y\rangle,\left\langle\langle Y, X\rangle \in \mathcal{C}_{2}^{2 \alpha}([0, T] ; V)\right.\right.$. Therefore, the integrals on the second line of (6.1) can be interpreted as Young's integrals. We can approximate them as follows:

$$
\begin{aligned}
& \int_{s}^{t} Z_{r}\left\langle\langle X, Y\rangle_{r}=Z_{s} D W_{s, t}\left(Y_{s}\right) Y_{s, t}-2 Z_{s} \widehat{Y}_{s} \mathcal{D}^{(1)} Y_{s}^{\prime} \mathcal{W}_{s, t}+O\left(|t-s|^{3 \alpha}\right),\right. \\
& \int_{s}^{t} Z_{r}\left\langle\langle Y, X\rangle_{r}=Z_{s} D W_{s, t}\left(Y_{s}\right) Y_{s, t}-2 Z_{s} Y_{s}^{\prime} \widehat{Y}_{s} \mathcal{D}^{(2)} \mathcal{W}_{s, t}+O\left(|t-s|^{3 \alpha}\right),\right.
\end{aligned}
$$


and

$$
\int_{s}^{t} Z_{r} D^{2} W\left(r, Y_{r}\right) d\langle Y\rangle_{r}=Z_{s} D^{2} W\left(s, Y_{s}\right)+O\left(|t-s|^{3 \alpha}\right) .
$$

Notice that, by definition,

$$
\begin{aligned}
\langle Y\rangle_{s, t} & =Y_{s, t} \otimes Y_{s, t}-2 \mathbb{Y}_{s, t}=Y_{s, t} \otimes Y_{s, t}-2\left(\int_{s}^{t} Y_{r} \otimes d Y_{r}-Y_{s} \otimes Y_{s, t}\right) \\
& =Y_{s, t} \otimes Y_{s, t}-2\left(Y_{s} \otimes Y_{s, t}+Y_{s}^{\prime} Y_{s}^{\prime} \mathcal{W}_{s, t}-Y_{s} \otimes Y_{s, t}\right)+O\left(|t-s|^{3 \alpha}\right) .
\end{aligned}
$$

This allows us to write

$$
\int_{s}^{t} Z_{r}=Z_{s} D^{2} W\left(s, Y_{s}\right)\left[Y_{s, t} \otimes Y_{s, t}-2 Y_{s}^{\prime} Y_{s}^{\prime} \mathcal{W}_{s, t}\right]+O\left(|t-s|^{3 \alpha}\right)
$$

As we approximated all the integrals in (6.1), the proof of Theorem 6.1 is straightforward.

Proof of Theorem 6.1: Denote by LHS and RHS the left and right-hand side of equation (6.1) respectively. Recall equality (6.3), that is,

$$
\begin{aligned}
L H S= & Z_{s} W_{s, t}\left(Y_{s}\right)+Z_{s} D W_{s, t}\left(Y_{s}\right) Y_{s, t}+Z_{s} D W_{s}\left(Y_{s}\right) Y_{s, t}+\frac{1}{2} Z_{s} D^{2} W_{s}\left(Y_{s}\right)\left(Y_{s, t}, Y_{s, t}\right) \\
& +Z_{s}^{\prime} \widehat{Y}_{s} \mathcal{W}_{s, t}+Z_{s} D W_{s}\left(Y_{s}\right) Y_{s}^{\prime} \mathcal{W}_{s, t}+O\left(|t-s|^{3 \alpha}\right) .
\end{aligned}
$$

On the other hand, combining (6.4) - (6.8), we have

$$
\begin{aligned}
R H S= & Z_{s} W_{s, t}\left(Y_{s}\right)+Z_{s} D W_{s, t}\left(Y_{s}\right) Y_{s, t}+Z_{s} D W_{s}\left(Y_{s}\right) Y_{s, t}+\frac{1}{2} Z_{s} D^{2} W_{s}\left(Y_{s}\right)\left(Y_{s, t}, Y_{s, t}\right) \\
& +Z_{s}^{\prime} \widehat{Y}_{s} \mathcal{W}_{s, t}+Z_{s} D W_{s}\left(Y_{s}\right) Y_{s}^{\prime} \mathcal{W}_{s, t}+O\left(|t-s|^{3 \alpha}\right),
\end{aligned}
$$

as well. Since $\alpha \in\left(\frac{1}{3}, \frac{1}{2}\right]$, it follows that equality (6.1) holds for all $0 \leq s \leq t \leq T$.

6.2. RDEs with spatial parameters. Let $(W, \mathcal{W}) \in \mathscr{C}^{\alpha}\left([0, T] ; \mathcal{C}^{\beta_{3}}\left(\mathbb{R}^{d} ; \mathbb{R}^{d}\right)\right)$, and let $\mathbb{W}$ be given by (5.1). Assume Hypothesis 1. Then, due to Theorem 4.4, for any fixed $x \in \mathbb{R}^{d}$, the following equation

$$
Y_{t}(x)=x+\int_{0}^{t} W\left(d r, Y_{r}(x)\right)
$$

has a unique solution $Y(x)$ on $[0, T]$. In this section, by studying the gradient in $x$ of $Y_{t}(x)$, we will show that $Y_{t}(x)$ is invertible in $x$, and the inverse is controlled by $W$ as well.

In the next theorem, we follow the idea of Hu and Lê (2017) to show that $Y_{t}(x)$ is differentiable in $x$. Before presenting the theorem, we introduce some notations. Let $M$ be a $d \times d$ matrix. We define the operators $M^{L}, M^{M}:\left(\mathbb{R}^{d} \otimes \mathbb{R}^{d}\right)^{\otimes 2} \rightarrow \mathbb{R}^{d} \otimes \mathbb{R}^{d}$ as follows, for any $(A, B) \in\left(\mathbb{R}^{d} \otimes \mathbb{R}^{d}\right)^{2}$,

$$
M^{L}(A \otimes B)=M \cdot A \cdot B \text { and } M^{M}(A \otimes B)=A \cdot M^{M} \cdot B .
$$

For any $d \times d$ matrices $M_{1}, M_{2}$, we define the operator $\left\{M_{1}, M_{2}\right\}: \mathbb{R}^{d} \otimes \mathbb{R}^{d} \rightarrow \mathbb{R}$ by

$$
\left\{M_{1}, M_{2}\right\} A=\sum_{k_{1}, k_{2}, k_{3}} M_{1}^{k_{1} k_{2}} M_{2}^{k_{1} k_{3}} A^{k_{2} k_{3}} \text { for all } A \in \mathbb{R}^{d} \otimes \mathbb{R}^{d} .
$$

These operators appear when we approximate matrix-valued rough integrals. 
Theorem 6.4. Let $(W, \mathcal{W}) \in \mathscr{C}^{\alpha}\left([0, T] ; \mathcal{C}^{\beta_{3}}\left(\mathbb{R}^{d} ; \mathbb{R}^{d}\right)\right)$. Assume Hypothesis 1. Let $Y=\left\{Y_{t}(x), t \in[0, T], x \in \mathbb{R}^{d}\right\}$ be the unique solution to (6.9). Then for any $t \in$ $[0, T], Y_{t}$ is differentiable, and the gradient DY satisfies the following equation:

$$
D Y_{t}(x)=I+\int_{0}^{t} d F_{r}(x) D Y_{r}(x)
$$

where $I$ denotes the $d \times d$ identity matrix and $F(x)$ is a $d \times d$ matrix-valued function given by

$$
F_{t}(x):=\int_{0}^{t} D W\left(d r, Y_{r}(x)\right)
$$

that is defined in the sense of (6.2). Moreover, for every $t \in[0, T]$ and $x \in \mathbb{R}^{d}$, $D Y_{t}(x)$ is invertible, and its inverse $\left(D Y_{t}(x)\right)^{-1}=: M_{t}(x)$ satisfies the following equation:

$$
M_{t}(x)=I-\int_{0}^{t} M_{r}(x) d F_{r}(x)+\int_{0}^{t}\left[M_{r}(x)\right]^{L} d\langle F(x)\rangle_{r} .
$$

where $\langle F(x)\rangle_{r}$ is the quadratic compensator of $F(x)$, which is an $\left(\mathbb{R}^{d} \otimes \mathbb{R}^{d}\right)^{\otimes 2}$-valued $2 \alpha$-Hölder continuous function on $[0, t]$, and $\left[M_{r}(x)\right]^{L}:\left(\mathbb{R}^{d} \otimes \mathbb{R}^{d}\right)^{\otimes 2} \rightarrow \mathbb{R}^{d} \otimes \mathbb{R}^{d}$ is defined as in (6.10).

Proof: Fix $x \in \mathbb{R}^{d}$. Let $e$ be a unit vector in $\mathbb{R}^{d}$. For any $h \in(0,1)$, we write

$$
\eta_{t}^{h}:=\frac{1}{h}\left[Y_{t}(x+h e)-Y_{t}(x)\right]
$$

We claim that as $h \downarrow 0, \eta_{t}^{h}$ converges to the solution to the following equation

$$
\eta_{t}=e+\int_{0}^{t} d F_{r}(x) \eta_{r}=e+\int_{0}^{t} D W\left(d r, Y_{r}(x)\right) \eta_{r} .
$$

Firstly, we show that (6.14) has a unique solution. Notice that $F(x)$ is defined as a nonlinear rough integral. Then, by Proposition 3.12, $F(x)$ is controlled by $D W$ and thus by $W$. That is,

$$
F_{s, t}(x)=D W_{s, t}\left(Y_{s}(x)\right)+O\left(|t-s|^{2 \alpha}\right):=\left(\widehat{Y}_{s}(x) D\right) W_{s, t}+O\left(|t-s|^{2 \alpha}\right),
$$

where $\widehat{Y} .(x) D$ is considered as an $\alpha$-Hölder continuous function on $[0, T]$ taking values in $\mathcal{L}\left(\mathcal{C}^{\beta_{3}}\left(\mathbb{R}^{d} ; \mathbb{R}^{d}\right) ; \mathcal{L}\left(\mathbb{R}^{d} ; \mathbb{R}^{d}\right)\right)$. Here $\widehat{Y}$ is defined in (5.3). We can also directly define the operator $\widehat{Y}_{s}(x) D$ by the former expression. $D W_{s, t}\left(Y_{s}(x)\right)$ is just an approximation of the integral without the double integral term, thus the error is $O\left(|t-s|^{2 \alpha}\right)$. By Proposition 2.7 (ii), $F(x)$ can be interpreted as a linear rough path. Thus, equation (6.14) is a linear RDE. According to the theory of linear RDE (see e.g. Theorem 2 of Lejay, 2009), this equation has a unique solution.

On the other hand, by Corollary $4.8,\left\|\eta^{h}\right\|_{\alpha}$ is uniformly bounded in $h \in(0,1)$. As a consequence of the Arzelà-Ascoli theorem, there exists a sequence $\left\{h_{n}\right\}_{n \geq 1}$, such that, as $n \rightarrow \infty, h_{n} \downarrow 0$, and $\eta_{t}^{h_{n}}$ converges to some function $\eta_{t}$ in $\mathcal{C}^{\alpha^{\prime}}\left([0, T] ; \mathbb{R}^{d}\right)$ for any fixed $\alpha^{\prime} \in(0, \alpha)$. In addition, by the Sewing Lemma, $\eta^{h_{n}}$ satisfies the following estimate

$$
\eta_{s, t}^{h_{n}}=D W_{s, t}\left(Y_{s}(x)\right) \eta_{s}^{h_{n}}+D \mathcal{W}_{s, t}\left(Y_{s}(x), Y_{s}(x)\right)\left(\eta_{s}^{h_{n}}, \eta_{s}^{h_{n}}\right)+O\left(|t-s|^{3 \alpha}\right)+O\left(h_{n}\right),
$$

for all $0 \leq s<t \leq T$. Let $n \rightarrow \infty$. The estimate (6.15) implies that $\eta_{t}$ satisfies the $\mathrm{RDE}$ (6.14). Therefore, $D Y_{t}(x)$ exists and is the unique solution to (6.12). 
To prove the invertibility of $D Y_{t}(x)$, we follow Stroock's idea (see Chapter 8 of Stroock, 1983). Let $M_{t}(x)$ be the unique solution to the linear RDE (6.13). By (2.16) and Itô's formula for linear rough paths (see e.g. Theorem 3.4 of Keller and Zhang, 2016), we can deduce the following equation:

$$
\begin{aligned}
D Y_{t}(x) M_{t}(x)= & +\int_{0}^{t} d F_{r}(x) D Y_{r}(x) M_{r}(x)-\int_{0}^{t} D Y_{r}(x) M_{r}(x) d F_{r}(x) \\
& +\int_{0}^{t}\left[D Y_{r}(x) M_{r}(x)\right]^{L} d\langle F(x)\rangle_{r}-\int_{0}^{t}\left[D Y_{r}(x) M_{r}(x)\right]^{M} d\langle F(x)\rangle_{r},
\end{aligned}
$$

where $\left[D Y_{r}(x) M_{r}(x)\right]^{M}$ is a linear operator on $\left(\mathbb{R}^{d} \otimes \mathbb{R}^{d}\right)^{\otimes 2}$ defined as in (6.10). Notice that $D Y_{t}(x) M_{t}(x) \equiv I$ solves this equation. Thus the uniqueness of linear RDEs implies that $M_{t}=\left(D Y_{t}\right)^{-1}$.

Remark 6.5. By taking further spatial derivatives on both sides of (6.12) and (6.13), we can show that $D Y_{t}$ and $M_{t}$ are both twice spatial differentiable with locally bounded derivatives. On the other hand, since Theorem 6.4 shows that $D Y_{t}(x)$ is invertible in $x$ for all $(t, x) \in[0, T] \times \mathbb{R}^{d}$, by the implicit function theorem, we deduce that for any fixed $t \in[0, T], Y_{t}$ has an inverse $Z_{t}$ such that $Z_{t}\left(Y_{t}(x)\right)=$ $Y_{t}\left(Z_{t}(x)\right)=x$.

In the next lemma, we prove that fix $x \in \mathbb{R}^{d}, Z(x)$ is controlled by $W$.

Lemma 6.6. Let $Y(x)=\left\{Y_{t}(x), t \in[0, T]\right\}$ be the solution to the $R D E$ (6.9), and let $Z_{t}=Y_{t}^{-1}$ be the inverse of $Y_{t}$. Fix $x \in \mathbb{R}^{d}$. Then $Z(x)$ is controlled by $W$.

Proof: Recall that for any $t \in[0, T], Z_{t}$ is the inverse of $Y_{t}$ and $D Y_{t} M_{t}=I$. Therefore, we can deduce that

$$
I=D x=D Y_{t}\left(Z_{t}(x)\right)=D Y_{t}\left(Z_{t}(x)\right) D Z_{t}(x) .
$$

This yields that

$$
D Z_{t}(x)=M_{t}\left(Z_{t}(x)\right)
$$

Fix $(t, x) \in(0, T] \times \mathbb{R}^{d}$. Let $y=Z_{t}(x)$. Then $x=Y_{t}(y)$. Notice that a similar argument as in Theorem 6.4 implies that $M_{t}(x)$ is differentiable in $x$ and the derivative is locally bounded. Thus by Taylor's Theorem 2.11, the following equality holds for all $s \in[0, t)$

$$
\begin{aligned}
Z_{s, t}(x) & =Z_{s}\left(Y_{s}(y)\right)-Z_{s}\left(Y_{t}(y)\right) \\
& =-D Z_{s}\left(Y_{s}(y)\right) Y_{s, t}(y)+O\left(|t-s|^{2 \alpha}\right) \\
& =-M_{s}\left(Z_{s}(x)\right) Y_{s, t}\left(Z_{s}(x)\right)+O\left(|t-s|^{2 \alpha}\right) .
\end{aligned}
$$

On the other hand, by Proposition 3.12, we have

$$
Y_{s, t}(x)=W_{s, t}\left(Y_{s}(x)\right)+O\left(|t-s|^{2}\right) .
$$

Combining above two inequalities, we can write

$$
Z_{s, t}(x)=-M_{s}\left(Z_{s}(x)\right) W_{s, t}(x)+O\left(|t-s|^{2 \alpha}\right) .
$$

Let $Z^{\prime}(x)=\left\{Z_{t}^{\prime}(x), t \in[0, T]\right\}$ where $Z_{t}^{\prime}(x): \mathcal{C}^{\beta_{3}}\left(\mathbb{R}^{d} ; \mathbb{R}^{d}\right) \rightarrow \mathbb{R}^{d}$ is given by

$$
Z_{t}^{\prime}(x) \Phi:=-M_{t}\left(Z_{t}(x)\right) \Phi(x) \text {. }
$$

Then it is easy to check that $Z_{s}^{\prime}(x) \in \mathcal{L}\left(\mathcal{C}^{\beta_{3}}\left(\mathbb{R}^{d} ; \mathbb{R}^{d}\right) ; \mathbb{R}^{d}\right)$, and thus $\left(Z(x), Z^{\prime}(x)\right) \in$ $\mathscr{D}_{W}^{2 \alpha}\left(\mathbb{R}^{d}\right)$. 
Remark 6.7. (i) One may find that $Z_{t}^{\prime}(x)=-D Z_{t}(x)$. But they are totally different objects. $Z_{t}^{\prime}(x)$ is the Gubinelli derivative that represents the proportional changing rate to $W$ of $Z_{t}(x)$ with respect to the time argument, while $D Z_{t}(x)$ is the spatial derivative of $Z_{t}$ for fixed $t$.

(ii) By taking derivative on both sides of (6.16), we have

$$
D^{2} Z_{t}(x)=D M_{t}\left(Z_{t}(x)\right) M_{t}\left(Z_{t}(x)\right) .
$$

Recall that $Y$ is the solution to $\operatorname{RDE}(6.9)$, thus

$$
Y_{s, t}(x)=W_{s, t}\left(Y_{s}(x)\right)+\mathbb{W}_{s, t}\left(Y_{s}(x), Y_{s}(x)\right)+O\left(|t-s|^{3 \alpha}\right) .
$$

This allow us to deduce an estimate, which is more precise than (6.17) and will be used in Section 6.3 below. We start with the following equation

$$
\begin{aligned}
Z_{s, t}(x)= & -M_{s}\left(Z_{t}(x)\right) Y_{s, t}\left(Z_{t}(x)\right)-\frac{1}{2} D M_{s}\left(Z_{t}(x)\right) M_{s}\left(Z_{t}(x)\right) Y_{s, t}\left(Z_{t}(x)\right)^{\otimes 2} \\
& +O\left(|t-s|^{3 \alpha}\right) \\
= & -M_{s}\left(Z_{t}(x)\right) W_{s, t}\left(Y_{s}\left(Z_{t}(x)\right)\right)-M_{s}\left(Z_{t}(x)\right) \mathbb{W}_{s, t}\left(Y_{s}\left(Z_{t}(x)\right), Y_{s}\left(Z_{t}(x)\right)\right) \\
& -\frac{1}{2} D M_{s}\left(Z_{t}(x)\right) M_{s}\left(Z_{t}(x)\right) Y_{s, t}\left(Z_{t}(x)\right)^{\otimes 2}+O\left(|t-s|^{3 \alpha}\right) .
\end{aligned}
$$

Notice that

$M_{s}\left(Z_{t}(x)\right) \mathbb{W}_{s, t}\left(Y_{s}\left(Z_{t}(x)\right), Y_{s}\left(Z_{t}(x)\right)\right)-M_{s}\left(Z_{s}(x)\right) \mathbb{W}_{s, t}(x, x)=O\left(|t-s|^{3 \alpha}\right)$

and

$$
\begin{aligned}
& M_{s}\left(Z_{t}(x)\right) W_{s, t}\left(Y_{s}\left(Z_{t}(x)\right)\right)-M_{s}\left(Z_{s}(x)\right) W_{s, t}\left(Y_{s}\left(Z_{s}(x)\right)\right) \\
= & D M_{s}\left(Z_{s}(x)\right) Z_{s, t}(x) W_{s, t}\left(Y_{s}\left(Z_{t}(x)\right)\right) \\
& +M_{s}\left(Z_{s}(x)\right) D W_{s, t}\left(Y_{s}\left(Z_{s}(x)\right)\right) D Y_{s}\left(Z_{s}(x)\right) Z_{s, t}(x)+O\left(|t-s|^{2 \alpha}\right) \\
= & -D M_{s}\left(Z_{s}(x)\right) M_{s}\left(Z_{s}(x)\right) W_{s, t}(x)^{\otimes 2} \\
& -M_{s}\left(Z_{s}(x)\right) D W_{s, t}(x) D Y_{s}\left(Z_{s}(x)\right) M_{s}\left(Z_{s}(x)\right) W_{s, t}(x)+O\left(|t-s|^{2 \alpha}\right),
\end{aligned}
$$

where for all $i=1,2, \ldots, d$,

$$
\begin{aligned}
& {\left[D M_{s}\left(Z_{s}(x)\right) M_{s}\left(Z_{s}(x)\right) W_{s, t}(x)^{\otimes 2}\right]^{i} } \\
= & \sum_{k_{1}, k_{2}, k_{3}=1}^{d} \frac{\partial M^{i k_{2}}(x)}{\partial x_{k_{1}}}\left(Z_{s}(x)\right) M_{s}^{k_{1} k_{3}}\left(Z_{s}(x)\right) W_{s, t}^{k_{2}}(x) W_{s, t}^{k_{3}} .
\end{aligned}
$$

Therefore, combining formulas (6.18) - (6.20), we have

$$
\begin{aligned}
Z_{s, t}(x)= & \frac{1}{2} D M_{s}\left(Z_{s}(x)\right) M_{s}\left(Z_{s}(x)\right) W_{s, t}(x)^{\otimes 2}+M_{s}\left(Z_{s}(x)\right) D W_{s, t}(x) W_{s, t}(x) \\
& -M_{s}\left(Z_{s}(x)\right) W_{s, t}(x)-M_{s}\left(Z_{s}(x)\right) \mathbb{W}_{s, t}(x, x)+O\left(|t-s|^{3 \alpha}\right) .
\end{aligned}
$$

6.3. Rough partial differential equations. Let $\mathcal{C}_{\text {loc }}^{3}\left(\mathbb{R}^{d} ; \mathbb{R}\right)$ be the space of functions that are locally bounded and have locally bounded first, second and third derivatives. Suppose that $h \in \mathcal{C}_{\text {loc }}^{3}\left(\mathbb{R}^{d} ; \mathbb{R}\right)$. In this section, we will show that $u=\left\{u(t, x)=h\left(Z_{t}(x)\right),(t, x) \in[0, T] \times \mathbb{R}^{d}\right\}$, where $Z_{t}(x)$ is defined in Section 6.1, is a solution to equation (1.7). Moreover, the solution is unique if $h \in \mathcal{C}_{\text {loc }}^{4}\left(\mathbb{R}^{d} ; \mathbb{R}\right)$ ). 
Definition 6.8. Let $(W, \mathcal{W}) \in \mathscr{C}^{\alpha}\left([0, T] ; \mathcal{C}^{\beta_{3}}\left(\mathbb{R}^{d} ; \mathbb{R}^{d}\right)\right)$, let $\mathbb{W}:[0, T]^{2} \times\left(\mathbb{R}^{d}\right)^{2} \rightarrow \mathbb{R}^{d}$ be given by (5.1), and let $h$ be a real-valued function on $\mathbb{R}^{d}$. A function $u=$ $\left\{u(t, x),(t, x) \in[0, T] \times \mathbb{R}^{d}\right\}$ is called a solution to equation (1.7) with initial condition $h$, if the following properties are satisfied:

(i) $u(0, x)=h(x)$ for all $x \in \mathbb{R}^{d}$.

(ii) $u$ is twice spatially differentiable everywhere, and $D u(\cdot, x)$ is controlled by $W$ for all $x \in \mathbb{R}^{d}$.

(iii) The following equality is true for all $(t, x) \in[0, T] \times \mathbb{R}^{d}$

$$
\begin{aligned}
u(t, x)= & h(x)-\int_{0}^{t} D u(r, x) W(d r, x)+\frac{1}{2} \int_{0}^{t} D u(r, x) d\left\langle\langle D W(x), W(x)\rangle_{r}\right. \\
& +\frac{1}{2} \int_{0}^{t} D u(r, x) d\left\langle\langle W(x), D W(x)\rangle_{r}+\frac{1}{2} \int_{0}^{t} D^{2} u(r, x) d\langle W(x)\rangle_{r},\right.
\end{aligned}
$$

where the first integral is defined as follows,

$$
\int_{0}^{t} D u(r, x) W(d r, x):=\left.\int_{0}^{t} D u(r, x) d W_{r}(\xi)\right|_{\xi=x},
$$

the quadratic compensators

and

$$
\begin{aligned}
& \left\langle\langle D W(x), W(x)\rangle_{s, t}:=\left.\langle\langle D W, W\rangle\rangle_{s, t}\left(\xi_{1}, \xi_{2}\right)\right|_{\left(\xi_{1}, \xi_{2}\right)=(x, x)},\right. \\
& \left\langle\langle W(x), D W(x)\rangle_{s, t}:=\left\langle\left.\langle W, D W\rangle_{s, t}\left(\xi_{1}, \xi_{2}\right)\right|_{\left(\xi_{1}, \xi_{2}\right)=(x, x)},\right.\right.
\end{aligned}
$$

$$
\langle W(x)\rangle_{s, t}:=\left.\langle W\rangle_{s, t}\left(\xi_{1}, \xi_{2}\right)\right|_{\left(\xi_{1}, \xi_{2}\right)=(x, x)}
$$

are defined by (2.15), (2.17) and (2.18) respectively, $D^{2} u(r, x)$ is considered as a linear operator from $\mathbb{R}^{d} \otimes \mathbb{R}^{d} \rightarrow \mathbb{R}$, that is

$$
D^{2} u(t, x) M=\sum_{i, j=1}^{d} \frac{\partial^{2} u(t, x)}{\partial x_{i} \partial x_{j}} M^{i j}
$$

for any $d \times d$ matrix $M=\left(M^{i j}\right)_{i, j=1}^{d}$, and the last three integrals are in Young's sense.

In the next theorem, we will show that $h\left(Z_{t}\right)$, where $Z_{t}$ is defined as in Lemma 6.6, is a solution to equation (1.7).

Theorem 6.9. Let $(W, \mathcal{W}) \in \mathscr{C}^{\alpha}\left([0, T] ; \mathcal{C}^{\beta_{3}}\left(\mathbb{R}^{d} ; \mathbb{R}^{d}\right)\right)$, and let $\mathbb{W}$ be given by $(5.1)$. Assume Hypothesis 1. Let $Y$ be the solution to the equation (6.9), and let $Z_{t}=Y_{t}^{-1}$ for all $t \in[0, T]$. Suppose that $h \in \mathcal{C}_{\text {loc }}^{3}\left(\mathbb{R}^{d} ; \mathbb{R}\right)$. Then, $u(t, x)=h\left(Z_{t}(x)\right)$ is a solution to (1.7) in the sense of Definition 6.8.

Proof: We prove this theorem by checking every property in Definition 6.8. By assumption, we know that $u(0, x)=h\left(Z_{0}(x)\right)=h(x)$. In addition, since $h \epsilon$ $\mathcal{C}_{\text {loc }}^{3}\left(\mathbb{R}^{d} ; \mathbb{R}\right)$ and $Z_{t}(x)$ is twice spatial differentiable, we can show that

$$
D\left[h\left(Z_{t}(x)\right)\right]=(D h)\left(Z_{t}(x)\right) M_{t}\left(Z_{t}(x)\right)
$$

and

$$
D^{2}\left[h\left(Z_{t}(x)\right)\right]=\left(D^{2} h\right)\left(Z_{t}(x)\right) M_{t}\left(Z_{t}(x)\right)^{2}+(D h)\left(Z_{t}(x)\right) D M_{t}\left(Z_{t}(x)\right),
$$

where $(D h)\left(Z_{t}(x)\right) D M_{t}(x)$ is a $d \times d$ matrix with components

$$
\left[(D h)\left(Z_{t}(x)\right) D M_{t}\left(Z_{t}(x)\right)\right]^{i j}=\sum_{k=1}^{d} \frac{\partial}{\partial x_{k}} h\left(Z_{t}(x)\right) \frac{\partial}{\partial x_{j}} M^{k i}\left(Z_{t}(x)\right) .
$$


Recall that $M_{t}(x)$ is the solution to the linear RDE (6.13). Then we can write

$$
M_{s, t}(x)=-M_{s}(x) F_{s, t}(x)+O\left(|t-s|^{2 \alpha}\right)=-M_{s}(x) D W_{s, t}\left(Y_{s}(x)\right)+O\left(|t-s|^{2 \alpha}\right) .
$$

Combining this fact with (6.17), we can deduce that

$$
\begin{aligned}
M_{t}\left(Z_{t}(x)\right) & -M_{s}\left(Z_{s}(x)\right)=M_{t}\left(Z_{t}(x)\right)-M_{s}\left(Z_{t}(x)\right)+M_{s}\left(Z_{t}(x)\right)-M_{s}\left(Z_{s}(x)\right) \\
= & M_{s, t}\left(Z_{t}(x)\right)-D M_{s}\left(Z_{s}(x)\right) Z_{s, t}(x)+O\left(|t-s|^{2 \alpha}\right) \\
= & M_{s, t}\left(Z_{s}(x)\right)+\left[M_{s, t}\left(Z_{t}(x)\right)-M_{s, t}\left(Z_{s}(x)\right)\right] \\
& -D M_{s}\left(Z_{s}(x)\right) M_{s}\left(Z_{s}(x)\right) W_{s, t}(x)+O\left(|t-s|^{2 \alpha}\right) \\
= & -M_{s}\left(Z_{s}(x)\right) D W_{s, t}(x)-D M_{s}\left(Z_{s}(x)\right) M_{s}\left(Z_{s}(x)\right) W_{s, t}(x)+O\left(|t-s|^{2 \alpha}\right) .
\end{aligned}
$$

Let $M_{t}^{\prime}\left(Z_{t}(x)\right): \mathcal{C}^{\beta_{3}}\left(\mathbb{R}^{d} ; \mathbb{R}^{d}\right) \rightarrow \mathbb{R}^{d} \otimes \mathbb{R}^{d}$ be given by

$$
M_{t}^{\prime}\left(Z_{t}(x)\right) \Phi:=-M_{t}\left(Z_{t}(x)\right) D \Phi(x)-D M_{t}\left(Z_{t}(x)\right) M_{t}\left(Z_{t}(x)\right) \Phi(x),
$$

where

$$
\left[D M_{t}\left(Z_{t}(x)\right) M_{t}\left(Z_{t}(x)\right) \Phi(x)\right]^{i j}=\sum_{k_{1}, k_{2}} \frac{\partial}{\partial x_{k_{1}}} M_{t}^{i j}\left(Z_{t}(x)\right) M_{t}^{k_{1} k_{2}}\left(Z_{t}(x)\right) \Phi^{k_{2}}(x)
$$

for any $(t, x) \in[0, T] \times \mathbb{R}^{d}$. We can show that

$$
M^{\prime}(Z(x)) \in \mathcal{C}^{\alpha}\left([0, T] ; \mathcal{L}\left(\mathcal{C}^{\beta_{3}}\left(\mathbb{R}^{d} ; \mathbb{R}^{d}\right) ; \mathbb{R}^{d} \otimes \mathbb{R}^{d}\right)\right) .
$$

Thus formulas (6.25) - (6.27) imply that

$$
\left(M(Z(x)), M^{\prime}(Z(x))\right) \in \mathscr{D}_{W}^{2 \alpha}\left(\mathcal{L}\left(\mathcal{C}^{\beta_{3}}\left(\mathbb{R}^{d} ; \mathbb{R}^{d}\right) ; \mathbb{R}^{d} \otimes \mathbb{R}^{d}\right)\right) .
$$

In a similar way, recalling (6.24) and (6.25), we can also deduce that

$$
\begin{aligned}
& D\left[h\left(Z_{t}(x)\right)\right]-D\left[h\left(Z_{s}(x)\right)\right]=(D h)\left(Z_{t}(x)\right) M_{t}\left(Z_{t}(x)\right)-(D h)\left(Z_{s}(x)\right) M_{t}\left(Z_{s}(x)\right) \\
= & (D h)\left(Z_{t}(x)\right)\left[M_{t}\left(Z_{t}(x)\right)-M_{s}\left(Z_{s}(x)\right)\right]+\left[(D h)\left(Z_{t}(x)\right)-D h\left(Z_{s}(x)\right)\right] M_{s}\left(Z_{s}(x)\right) \\
= & (D h)\left(Z_{s}(x)\right)\left[-M_{s}\left(Z_{s}(x)\right) D W_{s, t}(x)-D M_{s}\left(Z_{s}(x)\right) M_{s}\left(Z_{s}(x)\right) W_{s, t}(x)\right] \\
& -D^{2} h\left(Z_{s}(x)\right) M_{s}\left(Z_{s}(x)\right) W_{s, t}(x) M_{s}\left(Z_{s}(x)\right)+O\left(|t-s|^{2 \alpha}\right) .
\end{aligned}
$$

As a consequence, $D(h(Z(x))) \in \mathscr{D}_{W}^{2 \alpha}\left(\mathbb{R}^{d}\right)$ where the Gubinelli derivative

$$
[D h(Z(x))]^{\prime}: \mathcal{C}^{\beta_{3}}\left(\mathbb{R}^{d} ; \mathbb{R}^{d}\right) \rightarrow \mathbb{R}^{d}
$$

is given by

$$
\begin{aligned}
{[D(h(Z(x)))]^{\prime} \Phi=} & -(D h)\left(Z_{t}(x)\right) D M_{t}\left(Z_{t}(x)\right) M_{t}\left(Z_{t}(x)\right) \Phi(x) \\
& -(D h)\left(Z_{t}(x)\right) M_{t}\left(Z_{t}(x)\right) D \Phi(x) \\
& -\left(D^{2} h\right)\left(Z_{t}(x)\right) M_{t}\left(Z_{t}(x)\right) \Phi(x) M_{t}\left(Z_{t}(x)\right) .
\end{aligned}
$$

As a consequence, properties (i) and (ii) of Definition 6.8 are satisfied.

In the next step, we will prove equality (6.23) by a similar argument as in Theorem 6.1. For any $0 \leq s \leq t \leq T$, as a consequence of Taylor's Theorem 2.11, we can write

$$
\begin{aligned}
h\left(Z_{t}(x)\right)-h\left(Z_{s}(x)\right) & =(D h)\left(Z_{s}(x)\right) Z_{s, t}(x)+\frac{1}{2}\left(D^{2} h\right)\left(Z_{s}(x)\right) Z_{s, t}(x)^{\otimes 2}+O\left(|t-s|^{3 \alpha}\right) \\
& :=I_{1}+I_{2}+O\left(|t-s|^{3 \alpha}\right) .
\end{aligned}
$$


By (6.22), we have

$$
\begin{aligned}
I_{1}= & -(D h)\left(Z_{s}(x)\right) M_{s}\left(Z_{s}(x)\right) W_{s, t}(x)-(D h)\left(Z_{s}(x)\right) M_{s}\left(Z_{s}(x)\right) \mathbb{W}(x, x) \\
& +\frac{1}{2}(D h)\left(Z_{s}(x)\right) D M_{s}\left(Z_{s}(x)\right) M_{s}\left(Z_{s}(x)\right) W_{s, t}(x)^{\otimes 2} \\
& +(D h)\left(Z_{s}(x)\right) M_{s}\left(Z_{s}(x)\right) D W_{s, t}(x) W_{s, t}(x)+O\left(|t-s|^{3 \alpha}\right),
\end{aligned}
$$

and

$$
I_{2}=\frac{1}{2}\left[\left(D^{2} h\right)\left(Z_{s}(x)\right) M_{s}\left(Z_{s}(x)\right) W_{s, t}(x)\right] \cdot\left[M_{s}\left(Z_{s}(x)\right) W_{s, t}(x)\right]+O\left(|t-s|^{3 \alpha}\right),
$$

where

$$
\begin{aligned}
(D h) & \left(Z_{s}(x)\right) D M_{s}\left(Z_{s}(x)\right) M_{s}\left(Z_{s}(x)\right) W_{s, t}(x)^{\otimes 2} \\
= & \sum_{k_{1}, \ldots, k_{4}=1}^{d} \frac{\partial h}{\partial x_{k_{1}}}\left(Z_{s}(x)\right) \frac{\partial M_{s}^{k_{1} k_{2}}}{\partial k_{3}}\left(Z_{s}(x)\right) M_{s}^{k_{3} k_{4}}\left(Z_{s}(x)\right) W_{s, t}^{k_{2}}(x) W_{s, t}^{k_{4}}(x) .
\end{aligned}
$$

Recall that $D[h(Z(x))]$ is controlled by $W$ with Gubinelli derivative given by (6.28). Due to Theorem 2.4, the integral $\int_{s}^{t} D\left[h\left(Z_{r}(x)\right)\right] W(d r, x)$ is well-defined and it can be approximated as follows

$$
\begin{aligned}
\int_{s}^{t} D[ & \left.h\left(Z_{r}(x)\right)\right] W(d r, x)=(D h)\left(Z_{s}(x)\right) M_{s}\left(Z_{s}(x)\right) W_{s, t}(x) \\
& -\left\{\left(D^{2} h\right)\left(Z_{s}(x)\right) M_{s}\left(Z_{s}(x)\right), M_{s}\left(Z_{s}(x)\right)\right\} \mathcal{W}_{s, t}(x, x) \\
& -(D h)\left(Z_{s}(x)\right) M_{s}\left(Z_{s}(x)\right) \mathbb{W}_{s, t}^{*}(x, x) \\
& -(D h)\left(Z_{s}(x)\right) D M_{s}\left(Z_{s}(x)\right) M_{s}\left(Z_{s}(x)\right) \mathcal{W}_{s, t}(x, x)+O\left(|t-s|^{3 \alpha}\right),
\end{aligned}
$$

where $\left\{\left(D^{2} h\right)\left(Z_{s}(x)\right) M_{s}\left(Z_{s}(x)\right), M_{s}\left(Z_{s}(x)\right)\right\}$ is defined as in (6.11),

$$
\mathbb{W}_{s, t}^{*}(x, x)=\int_{s}^{t} D W_{s, r}(x) W(d r, x)=\mathcal{D}^{(1)} \mathcal{W}_{s, t}(x, x)
$$

and

$$
\begin{aligned}
(D h) & \left(Z_{s}(x)\right) D M_{s}\left(Z_{s}(x)\right) M_{s}\left(Z_{s}(x)\right) \mathcal{W}_{s, t}(x, x) \\
\quad= & \sum_{k_{1}, \ldots, k_{4}=1}^{d} \frac{\partial h}{\partial x_{k_{1}}}\left(Z_{s}(x)\right) \frac{\partial M_{s}^{k_{1} k_{3}}}{\partial x_{k_{2}}}\left(Z_{s}(x)\right) M_{s}^{k_{2} k_{4}}\left(Z_{s}(x)\right) \mathcal{W}_{s, t}^{k_{3} k_{4}}(x, x)
\end{aligned}
$$

Taking into account Definition 2.9 and Remark 2.10, we can write

$$
\begin{gathered}
\langle W(x)\rangle_{s, t}=W_{s, t}(x) \otimes W_{s, t}(x)-2 \mathcal{W}_{s, t}(x, x), \\
\left\langle\langle D W(x), W(x)\rangle_{s, t}=D W_{s, t}(x) W_{s, t}(x)-2 \mathbb{W}_{s, t}^{*}(x, x)\right.
\end{gathered}
$$

and

$$
\langle W(x), D W(x)\rangle_{s, t}=D W_{s, t}(x) W_{s, t}(x)-2 \mathbb{W}_{s, t}(x, x) .
$$


Therefore, combining (6.29) - (6.32), we have

$$
\begin{aligned}
h\left(Z_{t}(x)\right) & -h\left(Z_{s}(x)\right)+\int_{s}^{t} D\left[h\left(Z_{r}(x)\right)\right] W(d r, x) \\
= & \left.\frac{1}{2}(D h)\left(Z_{s}(x)\right) M_{s}\left(Z_{s}(x)\right)[\langle D W(x), W(x))\rangle_{s, t}+\langle\langle W(x), D W(x)\rangle\rangle_{s, t}\right] \\
& +\frac{1}{2}\left\{\left(D^{2} h\right)\left(Z_{s}(x)\right) M_{s}\left(Z_{s}(x)\right), M_{s}\left(Z_{s}(x)\right)\right\}\langle W(x)\rangle_{s, t} \\
& +\frac{1}{2}(D h)\left(Z_{s}(x)\right) D M_{s}\left(Z_{s}(x)\right) M_{s}\left(Z_{s}(x)\right)\langle W(x)\rangle_{s, t}+O\left(|t-s|^{3 \alpha}\right) .
\end{aligned}
$$

On the other hand, by the theory of Young's integral, we can show that

$$
\begin{aligned}
& \int_{0}^{t} D\left[h\left(Z_{r}(x)\right)\right] d\left\langle\langle D W(x), W(x)\rangle_{r}+\int_{0}^{t} D\left[h\left(Z_{r}(x)\right)\right] d\left\langle\langle W(x), D W(x)\rangle_{r}\right.\right. \\
& +\int_{0}^{t} D^{2}\left[h\left(Z_{r}(x)\right)\right] d\langle W(x)\rangle_{r} \\
= & (D h)\left(Z_{s}(x)\right) M_{s}\left(Z_{s}(x)\right)\left[\langle D W(x), W(x)\rangle_{s, t}+\left\langle\langle W(x), D W(x)\rangle_{s, t}\right]\right. \\
& +\left\{\left(D^{2} h\right)\left(Z_{s}(x)\right) M_{s}\left(Z_{s}(x)\right), M_{s}\left(Z_{s}(x)\right)\right\}\langle W(x)\rangle_{s, t} \\
& +(D h)\left(Z_{s}(x)\right) D M_{s}\left(Z_{s}(x)\right) M_{s}\left(Z_{s}(x)\right)\langle W(x)\rangle_{s, t}+O\left(|t-s|^{3 \alpha}\right) .
\end{aligned}
$$

It follows that $(6.23)$ holds if $u(t, x)=h\left(Z_{t}(x)\right)$ for all $(t, x) \in[0, T] \times \mathbb{R}^{d}$.

In the next theorem, we will show that the solution is unique in the space $\mathcal{C}_{l o c}^{\alpha, 3}\left([0, T] \times \mathbb{R}^{d}\right)$ provided that $(W, \mathcal{W}) \in \mathcal{C}^{\alpha}\left([0, T] ; \mathcal{C}^{\boldsymbol{\beta}_{4}}\left(\mathbb{R}^{d} ; \mathbb{R}^{d}\right)\right)$ and $h \in$ $\mathcal{C}_{\text {loc }}^{4}\left(\mathbb{R}^{d} ; \mathbb{R}\right)$.

Theorem 6.10. Let $(W, \mathcal{W}) \in \mathscr{C}^{\alpha}\left([0, T] ; \mathcal{C}^{\beta_{3}}\left(\mathbb{R}^{d} ; \mathbb{R}^{d}\right)\right)$, and let $\mathbb{W}$ be given by (5.1). Assume Hypothesis 1. Let $h \in \mathcal{C}_{\text {loc }}^{4}\left(\mathbb{R}^{d} ; \mathbb{R}\right)$. The solution to the RPDE (1.7) exists and is unique in the space $\mathcal{C}_{\text {loc }}^{\alpha, 3}\left([0, T] \times \mathbb{R}^{d} ; \mathbb{R}\right)$.

Proof: Firstly, we show the existence of the equation (1.7) in the space $\mathcal{C}_{\text {loc }}^{\alpha, 3}([0, T] \times$ $\left.\mathbb{R}^{d} ; \mathbb{R}\right)$. Due to Theorem 6.9 , it suffice to show that $h(Z) \in \mathcal{C}_{l o c}^{\alpha, 3}\left([0, T] \times \mathbb{R}^{d} ; \mathbb{R}\right)$.

Notice that $D Z_{t}(x)=M_{t}\left(Z_{t}(x)\right), D^{2} Z_{t}(x)=D M_{t}\left(Z_{t}(x)\right) M_{t}\left(Z_{t}(x)\right)$, and

$$
\begin{aligned}
D^{3} Z_{t}\left(Z_{t}(x)\right)= & D^{2} M_{t}\left(Z_{t}(x)\right) M_{t}\left(Z_{t}(x)\right) M_{t}\left(Z_{t}(x)\right) \\
& +D M_{t}\left(Z_{t}(x)\right) D M_{t}\left(Z_{t}(x)\right) M_{t}\left(Z_{t}(x)\right)
\end{aligned}
$$

for all $(t, x) \in[0, T] \times \mathbb{R}^{d}$. Fix $x \in \mathbb{R}^{d}$, the functions $M_{t}(x), D M_{t}(x), D^{2} M_{t}(x)$ and $D^{3} M_{t}(x)$ are all solutions to corresponding linear RDEs driven by $\alpha$-Hölder linear rough paths. Thus $M_{t}(x), D M_{t}(x), D^{2} M_{t}(x)$ and $D^{3} M_{T}(x)$ are all $\alpha$ Hölder in time and locally bounded in space. Recall that $h \in \mathcal{C}_{\text {loc }}^{4}\left(\mathbb{R}^{d} ; \mathbb{R}\right)$. As a consequence $h\left(Z_{t}(x)\right), D\left[h\left(Z_{t}(x)\right)\right], D^{2}\left[h\left(Z_{t}(x)\right)\right]$ and $D^{3}\left[h\left(Z_{t}(x)\right)\right]$ are all $\alpha$ Hölder in time and locally bounded in space. In other words, we can conclude that $h(Z) \in \mathcal{C}_{\text {loc }}^{\alpha, 3}\left([0, T] \times \mathbb{R}^{d} ; \mathbb{R}\right)$.

In the next step, we will prove the uniqueness of the RPDE (1.7). Suppose that $u \in \mathcal{C}^{\alpha, 3}\left([0, T] \times \mathbb{R}^{d} ; \mathbb{R}\right)$ is a solution to $(1.7)$. Let $Y$ be the solution to the RDE (6.9). Then, by Taylor's Theorem 2.11, we can write

$$
\begin{aligned}
u\left(t, Y_{t}(x)\right)-u\left(s, Y_{s}(x)\right)= & u_{s, t}\left(Y_{s}(x)\right)+D u_{s, t}\left(Y_{s}(x)\right) Y_{s, t}+D u_{s}\left(Y_{s}(x)\right) Y_{s, t}(x) \\
& +\frac{1}{2} D^{2} u_{s}\left(Y_{s}(x)\right) Y_{s, t}(x)^{\otimes 2}+O\left(|t-s|^{3 \alpha}\right) .
\end{aligned}
$$


Notice that as a solution to (1.7), $u$ satisfies the following equality for all $x \in \mathbb{R}^{d}$,

$$
u_{s, t}(x)=-D u_{s}(x) W_{s, t}(x)+O\left(|t-s|^{2 \alpha}\right) .
$$

It follows that fix $x \in \mathbb{R}^{d}, u(x)$ is controlled by $W(x)$. As a consequence, $D u(x)$ is also controlled by $W(x)$ with the Gubinelli derivative $-D^{2} u_{s}(x)$. Therefore, the following estimate holds

$$
\begin{aligned}
& u_{s, t}\left(Y_{s}(x)\right)=-D u\left(s, Y_{s}(x)\right) W_{s, t}\left(Y_{s}(x)\right)+D^{2} u\left(s, Y_{s}(x)\right) \mathcal{W}_{s, t}\left(Y_{s}(x), Y_{s}(x)\right) \\
& \quad+\frac{1}{2} D u(s, x)\left[\left\langle D W\left(Y_{s}(x)\right), W\left(Y_{s}(x)\right)\right\rangle_{s, t}+\left\langle\left\langle W\left(Y_{s}(x)\right), D W\left(Y_{s}(x)\right)\right\rangle_{s, t}\right]\right. \\
& \quad+\frac{1}{2} D^{2} u(s, x)\left\langle D W\left(Y_{s}(x)\right)\right\rangle_{s, t}+O\left(|t-s|^{3 \alpha}\right) .
\end{aligned}
$$

In addition, recall that $Y$ is the solution to (6.9). Then, (6.34) implies that

$$
\begin{aligned}
D u_{s, t}\left(Y_{s}(x)\right) Y_{s, t}(x)= & -D^{2} u\left(s, Y_{s}(x)\right) W_{s, t}\left(Y_{s}(x)\right) W_{s, t}\left(Y_{s}(x)\right) \\
& -D u\left(s, Y_{s}(x)\right) D W_{s, t}\left(Y_{s}(x)\right) W_{s, t}\left(Y_{s}(x)\right)+O\left(|t-s|^{3 \alpha}\right) .
\end{aligned}
$$

Also, we have the following estimates

$$
\begin{aligned}
D u_{s}\left(Y_{s}(x)\right) Y_{s, t}(x)= & D u_{s}\left(Y_{s}(x)\right) W_{s, t}\left(Y_{s}(x)\right) \\
& +D u_{s}\left(Y_{s}(x)\right) \mathbb{W}_{s, t}\left(Y_{s}(x), Y_{s}(x)\right)+O\left(|t-s|^{3 \alpha}\right),
\end{aligned}
$$

and

$$
D^{2} u_{s}\left(Y_{s}(x)\right) Y_{s, t}(x)^{\otimes 2}=D^{2} u_{s}\left(Y_{s}(x)\right) W_{s, t}\left(Y_{s}(x)\right)^{\otimes 2}+O\left(|t-s|^{3 \alpha}\right) .
$$

Combining (6.33) - (6.37), we have

$$
u\left(t, Y_{t}(x)\right)-u\left(s, Y_{s}(x)\right)=O\left(|t-s|^{3 \alpha}\right) .
$$

Because $\alpha \in\left(\frac{1}{3}, \frac{1}{2}\right]$, it follows that $u\left(t, Y_{t}(x)\right) \equiv u\left(0, Y_{0}(x)\right)=h(x)$. In other words, $u(t, x)=u\left(t, Y_{t}\left(Z_{t}(x)\right)\right)=h\left(Z_{t}(x)\right)$ for all $(t, x) \in[0, T] \times \mathbb{R}^{d}$. This completes the proof of the theorem.

\section{Acknowledgement}

We would like to thank two anonymous referees who read our manuscript very carefully and provided numerous valuable and detailed comments.

\section{References}

Bailleul, I. and Catellier, R. Rough flows and homogenization in stochastic turbulence. J. Differential Equations, 263 (8), 4894-4928 (2017). MR3680942.

Bailleul, I. and Riedel, S. Rough flows. J. Math. Soc. Japan, 71 (3), 915-978 (2019). MR3984248.

Bailleul, I., Riedel, S., and Scheutzow, M. Random dynamical systems, rough paths and rough flows. J. Differential Equations, 262 (12), 5792-5823 (2017). MR3624539.

Besalú, M. and Nualart, D. Estimates for the solution to stochastic differential equations driven by a fractional Brownian motion with Hurst parameter $H \epsilon$ $\left(\frac{1}{3}, \frac{1}{2}\right)$. Stoch. Dyn., 11 (2-3), 243-263 (2011). MR2836524.

Brault, A. and Lejay, A. The non-linear sewing lemma II: Lipschitz continuous formulation. ArXiv Mathematics e-prints (2018). arXiv: 1810.11988. 
Brault, A. and Lejay, A. The non-linear sewing lemma I: weak formulation. Electron. J. Probab., 24, Paper No. 59, 24 (2019a). MR3978209.

Brault, A. and Lejay, A. The non-linear sewing lemma III: Stability and generic properties. HAL Preprint hal-2265268 (2019b).

Catellier, R. Rough linear transport equation with an irregular drift. Stoch. Partial Differ. Equ. Anal. Comput., 4 (3), 477-534 (2016). MR3538009.

Catellier, R. and Gubinelli, M. Averaging along irregular curves and regularisation of ODEs. Stochastic Process. Appl., 126 (8), 2323-2366 (2016). MR3505229.

Chouk, K. and Gubinelli, M. Nonlinear PDEs with modulated dispersion II: Korteweg-de Vries equation. ArXiv Mathematics e-prints (2014). arXiv: 1406.7675.

Chouk, K. and Gubinelli, M. Nonlinear PDEs with modulated dispersion I: Nonlinear Schrödinger equations. Comm. Partial Differential Equations, 40 (11), 2047-2081 (2015). MR3418825.

Davie, A. M. Differential equations driven by rough paths: an approach via discrete approximation. Appl. Math. Res. Express. AMRX, pp. Art. ID abm009, 40 (2008). MR2387018.

Feyel, D. and de La Pradelle, A. Curvilinear integrals along enriched paths. Electron. J. Probab., 11, no. 34, 860-892 (2006). MR2261056.

Flandoli, F., Gubinelli, M., and Priola, E. Well-posedness of the transport equation by stochastic perturbation. Invent. Math., 180 (1), 1-53 (2010). MR2593276.

Friz, P. K. and Hairer, M. A course on rough paths. Universitext. Springer, Cham (2014). ISBN 978-3-319-08331-5; 978-3-319-08332-2. MR3289027.

Gubinelli, M. Controlling rough paths. J. Funct. Anal., 216 (1), 86-140 (2004). MR2091358.

$\mathrm{Hu}, \mathrm{Y}$. and Lê, K. Nonlinear Young integrals and differential systems in Hölder media. Trans. Amer. Math. Soc., 369 (3), 1935-2002 (2017). MR3581224.

$\mathrm{Hu}$, Y., Lu, F., and Nualart, D. Feynman-Kac formula for the heat equation driven by fractional noise with Hurst parameter $H<1 / 2$. Ann. Probab., 40 (3), 10411068 (2012). MR2962086.

$\mathrm{Hu}, \mathrm{Y}$. and Nualart, D. Rough path analysis via fractional calculus. Trans. Amer. Math. Soc., 361 (5), 2689-2718 (2009). MR2471936.

$\mathrm{Hu}$, Y., Nualart, D., and Song, J. Feynman-Kac formula for heat equation driven by fractional white noise. Ann. Probab., 39 (1), 291-326 (2011). MR2778803.

Keller, C. and Zhang, J. Pathwise Itô calculus for rough paths and rough PDEs with path dependent coefficients. Stochastic Process. Appl., 126 (3), 735-766 (2016). MR3452811.

Lejay, A. On rough differential equations. Electron. J. Probab., 14, no. 12, 341-364 (2009). MR2480544.

Lejay, A. Global solutions to rough differential equations with unbounded vector fields. In Séminaire de Probabilités XLIV, volume 2046 of Lecture Notes in Math., pp. 215-246. Springer, Heidelberg (2012). MR2953350.

Lyons, T. Differential equations driven by rough signals. I. An extension of an inequality of L. C. Young. Math. Res. Lett., 1 (4), 451-464 (1994). MR1302388.

Lyons, T. and Qian, Z. System control and rough paths. Oxford Mathematical Monographs. Oxford University Press, Oxford (2002). ISBN 0-19-850648-1. MR2036784. 
Lyons, T. J. Differential equations driven by rough signals. Rev. Mat. Iberoamericana, 14 (2), 215-310 (1998). MR1654527.

Stroock, D. W. Some applications of stochastic calculus to partial differential equations. In Eleventh Saint Flour probability summer school-1981 (Saint Flour, 1981), volume 976 of Lecture Notes in Math., pp. 267-382. Springer, Berlin (1983). MR722984.

Young, L. C. An inequality of the Hölder type, connected with Stieltjes integration. Acta Math., 67 (1), 251-282 (1936). MR1555421.

Zanco, G. A brief introduction to rough paths. http://pub.ist.ac.at/ gzanco/ A_brief_introduction_to_rough_paths.pdf (2016).

Zeidler, E. Applied functional analysis. Main principles and their applications, volume 109 of Applied Mathematical Sciences. Springer-Verlag, New York (1995). ISBN 0-387-94422-2. MR1347692. 